\title{
TWO-DIMENSIONAL CUSPED INTERFACES
}

\author{
By \\ Daniel D. Joseph \\ John Nelson \\ Michael Renardy \\ and \\ Yuriko Renardy
}

IMA Preprint Series \# 624

February 1990 


\title{
TWO-DIMENSIONAL CUSPED INTERFACES
}

\author{
Daniel D. Joseph and John Nelson \\ Department of Aerospace Engineering and Mechanics \\ University of Minnesota \\ 110 Union Street SE \\ Minneapolis, MN 55455
}

\section{Michael Renardy and Yuriko Renardy}

Department of Mathematics and ICAM

Virginia Polytechnic Institute and State University

Blacksburg, VA 24061-0123

Two-dimensional cusped interfaces are line singularities of curvature. We create such cusps by rotating a cylinder half immersed in liquid. A liquid film is dragged out of the reservoir on one side and is plunged in at the other, where it forms a cusp at finite speeds, if the conditions are right. Both Newtonian and non-Newtonian fluids form cusps, but the transition from a rounded interface to a cusp is gradual in Newtonian liquids and sudden in non-Newtonian liquids. We present an asymptotic analysis near the cusp tip for the case of zero surface tension, and we make some remarks about the effects of a small surface tension. We also present the results of numerical simulations showing the development of a cusp. In those simulations, the fluid is filling an initially rectangular domain with a free surface on top. The fluid enters from both sides and is sucked out through a hole in the bottom. 


\section{Introduction}

Our photographs were produced in a four roll mill apparatus of the type introduced by G.I. Taylor to study the deformation of drops and bubbles in a pure straining flow. We built the apparatus with the same purpose in mind, but first put the cylinders into rotation in a partially filled apparatus whose configuration and orientation relative to gravity are obvious from the photographs. As a result, we discovered the rollers which were described by Joseph, Nguyen and Beavers [1984].

At that time, we noticed another entirely different flow regime, the two-dimensional cusped interfaces which we shall describe in this paper. At this point, we ask the reader to inspect the photographs. They show cusped, two-dimensional interfaces. No rounding can be detected, at least not on a visible length scale.

S. Richardson [1968] did an elegant analysis of two-dimensional bubbles in Stokes flow. He proved that the only possible line singularity of the interface between a viscous liquid and an inviscid two-dimensional bubble is a true cusp. For a cusp that opens on the negative $\mathrm{x}$-axis, his expression for the leading contribution to the stream function, expressed in polar coordinates, is

$$
\psi=\frac{\sigma}{2 \pi \mu} \mathrm{r} \log r \sin \phi
$$

Here $\sigma$ is the surface tension coefficient and $\mu$ is the viscosity. This formula shows that there is a point force $2 \sigma$ exerted by the free surface on the fluid and that the velocity near the origin is in the negative $\mathrm{x}$-direction. The velocity near the origin is infinite and, perhaps more seriously, the velocity gradient is not square integrable, leading to an infinite amount of energy dissipation. The free surface at leading order simply coincides with the negative $x$-axis; the actual opening of the cusp would have to be determined from higher order corrections. Such corrections have not been worked out. 
Evidently the main features of our experiments cannot be explained in terms of (1), since the velocity of the fluid is in the positive, rather than the negative, $\mathrm{x}$-direction, as shown in Figure 1. Moreover, the deviation of the interface from a flat surface, neglected by Richardson, is clearly an important feature of the experiments. The experiments also suggest that, although cusped free surfaces can appear in Newtonian fluids, they are easier to create in non-Newtonian fluids. The cusp does not appear at the slowest speeds; it forms more gradually in Newtonian than in nonNewtonian fluids. This is reminiscent of the transition from rounded to pointed ends in the bubble experiments of Rumscheidt and Mason [1961].

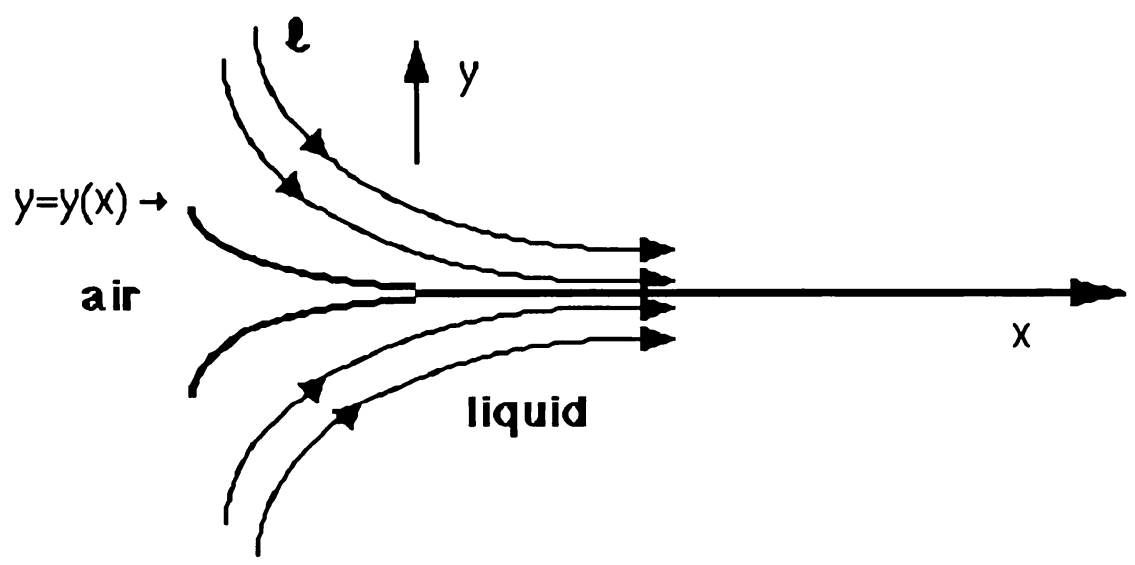

Figure 1. $\quad$ Flow past a cusp.

Our analysis takes a different point of view from Richardson's. We first analyze the local behavior near a cusp singularity in the absence of surface tension. In this case, we find an interface shape given at leading order by $y^{2}=-c x^{3}$ for Newtonian and linear viscoelastic fluids. The analysis can be extended to linear viscoelastic fluids; in that case the stresses at the cusp are less singular than in the Newtonian case. On the other hand, nonlinear effects in viscoelastic fluids are probably important near the stagnation point that must be present on a smooth interface. The build-up of extensional stresses near such a stagnation point is likely to favor cusping. In the presence of surface tension, our analysis is not valid close to the tip of the cusp, where the leading contribution (if there is a true cusp) should be given by Richardson's solution. As a measure of the importance of Richardson's solution, we calculate where the magnitude of the resulting velocity 
would be comparable to that of the macroscopically observed flow. For the surface tension parameters involved in the experiments, this is the case only at extremely small length scales, extending beyond the limits of the validity of Laplace's theory of surface tension. The issue was addressed by Lord Rayleigh [1890], who notes that “... the walls of a moderately small cavity certainly tend to collapse with a force measured by the constant surface tension of the liquid. The pressure in the cavity is first proportional to the surface tension and to the curvature of the walls. If this law held without limit, the consideration of an infinitely small cavity shows that the intrinsic pressure would be infinite in all liquids. Of course, the law really changes when the dimensions of the cavity are of the same order as the range of attractive forces, and the pressure in the cavity approaches a limit." We therefore believe that surface tension plays no major role in the experimentally observed flows and that the analysis given here describes the main features.

Richardson's result and ours may have some relevance for pointed bubbles, which are point singularities of the curvature. Such singularities have been described by Taylor [1934], Rumscheidt and Mason [1961], Taylor [1964], Grace [1971], Buckmaster [1972,1973], Acrivos and Lo [1978], Rallison and Acrivos [1978], Hinch and Acrivos [1979], and Sherwood [1981] for the case of Newtonian fluids. Buckmaster is the only author in this list who discussed Richardson's results. He comments that Richardson has “... shown that such discontinuities, if they exist, must be genuine cusps ..." He also expresses reservations about the relevance of twodimensional cusps: "On the one hand it is doubtful that pointed two-dimensional drops could be stable, and on the other there is a point force associated with corners-which a three-dimensional drop would not generate." These remarks appear to have halted further consideration of twodimensional cusps.

The existence of point singularities for axisymmetric bubbles in Newtonian fluids and the decision about whether these might be corner or cusped singularities still seems not to have been decided. 
There is a marked difference between the shape of air bubbles rising in Newtonian and non-Newtonian liquids, with a much stronger tendency toward cusping in the non-Newtonian case (see Figure 2).

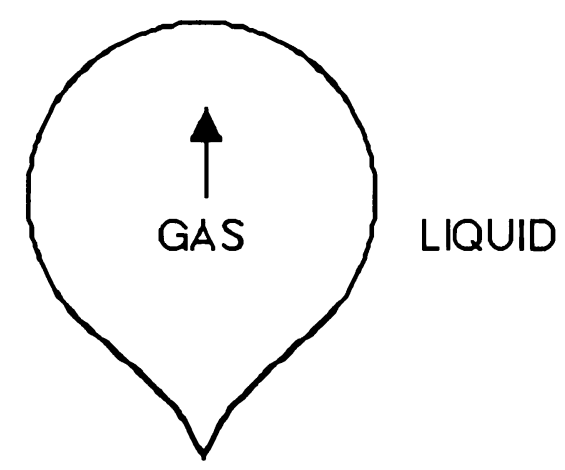

Figure 2. Gas bubble rising in a non-Newtonian liquid. Bubbles rising in Newtonian liquids do not tail. The shape of the bubble depends strongly on the bubble volume.

The flow at the trailing edge of a rising gas bubble is in a rough way analogous to flow near the cusp in our experiments, though there are obvious and perhaps not so obvious differences between streaming around axisymmetric and plane cusps. The tail shown in Figure 2 occurs only in non-Newtonian liquids and has been reported in experiments by Phillippoff [1937], Warshay, Bougsz, Johnson and Kintner [1959], Mhatre and Kintner [1959], Astarita and Apuzzo [1965], Barnett, Humphrey and Litt [1966], Calderbank [1967], Calderbank, Johnson and Loudon [1970], Leal, Skoog and Acrivos [1971] and Zana and Leal [1978]. Again, it is not certain that the tail can form a true cusp. Astarita and Apuzzo note that "The bubble is ... unexpected; it is clearly prolate and the lower pole is markedly cuspoidal." Hassager [1985] reports experiments where the bubble is not axisymmetric but flattened; it appears to have a straight edge at the trailing end.

We present our experimental results in the following section. Section 3 contains the local analysis of fluid behavior near a cusp. In Section 4, we show some numerical simulations using the FIDAP package. The flow there involves a fluid entering from both the right and left and bounded by a free surface on top. The fluid is being sucked downward. The numerical results are consistent with the interpretation that a singularity in the free surface is developing if the surface 
tension is small enough. For larger values of surface tension, a steady state with a smooth interface is reached instead. The final section of the paper gives a summary of the results.

\section{Experiments}

Two equal cylinders whose centerlines lie in the same plane $\mathrm{z}=$ const., perpendicular to gravity, are immersed in liquid approximately up to their centerline. Then the cylinders are put into counter rotation with the same speed. There are two possibilities; the liquid is drawn up at the outside of the cylinders and forced down in the center, or vice versa, as in Figures 3, 4, 5 and 6. Typically more than half of each cylinder is coated with a liquid of relatively uniform thickness. The thickness and covering of the arc of the coating liquid depends on the viscosity of the liquid and the speed of the cylinder.

Cusps form in some liquids and not in others. Once a cusp forms it will persist and sharpen as the angular velocity is increased. Liquids which do not cusp have clearly rounded ends as in Figures 8,10 and 12. Tables 1 and 2 list fluids which form cusps and Table 3 lists fluids which do not cusp at the angular speeds we attained. The fluids that do eventually cusp apparently do not do so at the slowest speed. It therefore appears that cusping is a threshold phenomenon characterized by a threshold parameter which in our experiments is a critical value of the angular velocity of the cylinders. Some specific values of this critical speed are given in the figure captions; to convert this rotation speed into a linear speed one has to multiply by the radius of the cylinder, which is $1.25 \mathrm{~cm}$. For non-Newtonian fluids, the critical value of the rotation speed is very distinct. Below this value the interface is round. At the critical speed the interface suddenly transforms to a cusp. The critical speed is not distinct for those Newtonian fluids for which a cusp was observed. At slow rotation speeds the interface is round. As the rotation speed increases, the shape of the interface changes from rounded to pointed in a continuous manner, until at what we define as the critical speed the interface appears to be a cusp. Once a cusp is formed, further increases in the angular velocity will only sharpen the cusp or end in an instability characterized by 
fingering. It is for this reason that the cusped interfaces shown in Figures 3, 4, 5, 6 and 7 are typical, independent of the angular velocity.

The liquids listed in Table 1 which do form apparently unambiguous cusps are nonNewtonian. They have non-zero normal stress differences and they all climb rotating rods. At the same time, these fluids are on the average more viscous than the Newtonian liquids which do, and those which do not, form cusps and are listed in Tables 2 and 3. We tested the liquids in Tables 2 and 3 and none of them will climb a rotating rod. Newtonian fluids which are too mobile will not cusp at the speeds we could attain in our apparatus and may never cusp because of turbulence.

\begin{tabular}{|l|c|c|c|}
\hline \multicolumn{1}{|c|}{ Fluid } & Viscosity $(\mathrm{p})$ & Density $\left(\mathrm{g} / \mathrm{cm}^{3}\right)$ & Surface Tension $(\mathrm{dyn} / \mathrm{cm})$ \\
\hline 1\% Aqueous Polyox & 61.2 & 1.000 & 48.4 \\
2\% Aqueous Polyox & 600 & 1.125 & 44.4 \\
M1 & 30.0 & 0.859 & 29.9 \\
Silicone Oil-12500cs & 122 & 0.975 & 21.5 \\
STP & 143 & 0.858 & 35.0 \\
TLA 510 & 220 & 0.868 & 31.1 \\
Honey & 109 & 1.40 & 69.3 \\
\hline
\end{tabular}

Table 1. Representative values of the viscosity and surface tension of cusping non-Newtonian liquids at temperatures in the neighborhood of $23^{\circ} \mathrm{C}$.

\begin{tabular}{|l|c|c|c|}
\hline \multicolumn{1}{|c|}{ Fluid } & Viscosity $(\mathrm{p})$ & Density $\left(\mathrm{g} / \mathrm{cm}^{3}\right)$ & Surface Tension (dyn/cm) \\
\hline Castor Oil & 8.15 & 0.960 & 35.1 \\
Glycerin & 8.30 & 1.265 & 63.3 \\
Silicone Oil-500cs & 4.86 & 0.971 & 21.1 \\
Silicone Oil-1000cs & 9.71 & 0.971 & 21.2 \\
Silicone Oil-5000cs & 48.6 & 0.971 & 25.7 \\
\hline
\end{tabular}

Table 2. Representative values of the viscosity and surface tension of cusping Newtonian liquids at temperatures in the neighborhood of $23^{\circ} \mathrm{C}$.

\begin{tabular}{|l|c|c|c|}
\hline \multicolumn{1}{|c|}{ Fluid } & Viscosity $(\mathrm{p})$ & Density $\left(\mathrm{g} / \mathrm{cm}^{3}\right)$ & Surface Tension $($ dyn $/ \mathrm{cm})$ \\
\hline SAE 30 Motor Oil & 2.80 & 0.886 & 35.0 \\
Safflower Oil & 0.469 & 0.920 & 23.0 \\
Silicone Oil-200cs & 1.94 & 0.970 & 21.0 \\
Soybean Oil & 0.489 & 0.922 & 25.7 \\
\hline
\end{tabular}

Table 3. Representative values of the viscosity and surface tension of Newtonian liquids that do not cusp at temperatures in the neighborhood of $23^{\circ} \mathrm{C}$.

We measured the critical value of the angular velocity of the cylinders for the transition from rounded to cusped ends in a number of liquid-air systems and in the two-liquid SAE 30 
motor oil and water system shown in Figures 8, 9, and 10. A dimensionless capillary number was calculated by the formula $\mathrm{Ca}=\mu \mathrm{U} / \sigma$, where $\mu$ is the viscosity, $\sigma$ the surface tension coefficient, and an estimate for the velocity $U$ is $\omega r$, where $\omega$ is the angular speed of the cylinder and $r$ is the radius of the cylinder. Since the actual value of $r$ at the cusp point is somewhat larger than the radius of the cylinder, our values of $\mathrm{Ca}$ are slightly too low. The value of $\mathrm{Ca}$ for SAE 30-water was computed using a surface tension constant of $9.22 \mathrm{dyn} / \mathrm{cm}$; the surface tension constant tabulated above is for SAE 30 against air.

\begin{tabular}{|l|c|c|}
\hline \multicolumn{1}{|c|}{ System } & Critical Angular Velocity (RPM) & $\mathrm{Ca}_{\mathrm{c}}$ \\
\hline Castor Oil & 113 & 3.43 \\
Glycerin & 128 & 2.20 \\
Silicone Oil-500cs & 75 & 2.26 \\
Silicone Oil-1000cs & 41 & 2.46 \\
Silicone Oil-5000cs & 26 & 7.77 \\
SAE 30 Motor Oil-Water & 51 & 2.03 \\
\hline \multicolumn{1}{|c|}{ Non-Newtonian Fluids: } & & 1.15 \\
\hline Honey & 5.58 & 8.61 \\
Polyox (1\%) & 52 & 1.42 \\
Polyox (2\%) & 0.8 & 2.23 \\
Silicone Oil-12500cs & 3.0 & 0.32 \\
M1 & 2.4 & 1.55 \\
STP & 2.9 & \\
\hline
\end{tabular}

Table 4. Critical annular velocity and $\mathrm{Ca}_{c}$ values for the formation of a cusp.

For all Newtonian fluids, the measured values of $\mathrm{Ca}_{c}$ are in excess of 2. Some nonNewtonian fluids lead to lower values of $\mathrm{Ca}_{\mathrm{a}}$. However, this appearance may be deceiving. The "viscosity" used in computing $\mathrm{Ca}$ is the zero shear rate viscosity. The flows studied here are likely to involve significant extensional motion, and extensional viscosities of non-Newtonian fluids increase substantially with the rate of extension.

We tried to obtain two-dimensional cusped interfaces in a number of two-liquid systems. In general we do not expect to achieve cusped interfaces in two-liquid systems; the dynamics which would lead to a cusp in air will give rise to a fingering flow or rollers in a two-liquid system. In many of these two-liquid systems, rollers and fingering flows were described already by Joseph, Nguyen and Beavers [1984]. Rollers form when one of the two fluids is much more viscous than the other. For example, rollers form in STP-water and silicone oil-water systems 
with oils in excess of 1000p. Therefore, fluids like $12500 \mathrm{cs}$ silicone oil, STP and TLA 510 which give rise to cusps in air lead to rollers in water. On the other hand, $100 \mathrm{cs}$ silicone oil in water gives rise to the fingering of water into silicone oil at higher speeds and not to rollers.

For lower viscosity liquids the interface remains smooth at low speeds, while at higher speeds one liquid will finger into the other. In thinking about this, it is useful to imagine how the streamlines would look at a nearly cusped smooth surface. This is sketched in Figure 19. It is clear that the nose of the interface must be a stagnation point if the interface is not cusped. The internal motion of the fluid inside the nose is yet more complicated. It is being dragged toward the nose near the interface and must either be turned around by the pressure at the stagnation point into the double eddy or else finger into the fluid outside the nose. At higher speeds both these possibilities are realized intermittently.

There are evidently some pairs of liquids for which cusp solutions can be realized. One of these, motor oil and water, is shown in Figure 8, 9, and 10. The reader should notice that this cusped surface is not two-dimensional, but scalloped. The crests of the scallops are fingering sites for small water bubbles to enter the oil. The scalloping is a fairly common feature and it can be seen in the polyox-air interface shown in Figure 16(d). At high speeds the air at a cusped interface fingers into the liquid at these sites. Fingering always appears at scallop sites, really by definition because the scallop is what remains after the bubble has broken away. This kind of fingering leads to emulsions (cf. Section 5.3 in Joseph, Nguyen and Beavers [1984]). 


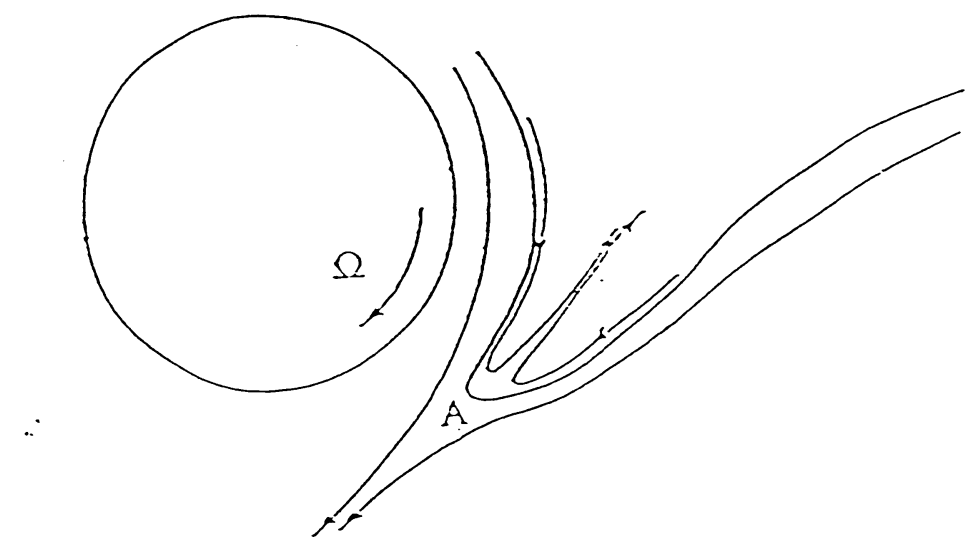

Figure 19. Distorted interface in a two-liquid system. The point $\mathrm{A}$ is a stagnation point or a cusp. When $\Omega=0$, each fluid covers one-half of the cylinder. We do not know' which nuid will finally be on the rod. There is nothing in our equations to tell us; contact angles are not enough.

\section{Theory}

We are going to assume that there is a cusp at the origin of the $x, y$-plane as shown in Figure 1.

The flow is assumed to be a small pertitation of uniform flow with velocity $U$ in the positive $x$-direction, and the free surface is a smail perturbation of the negative $x$-axis. Let $(u, v)$ denote the perturbations to the velocity and let $\mathrm{i}$ denote the position of the free surface that is located above the cusp. We first analyze Newtonian fluids with zero surface tension. We introduce a stream function $\psi$ by

$$
\mathrm{u}=\psi_{\mathrm{y}}, \mathrm{v}=-\psi_{\mathrm{x}}
$$

The positive $\mathrm{x}$-axis is a line of symmetry, we first look for antisymmetric stream functions, corresponding to symmetric flow fields. In polar coordinates, we then have the ansatz for the stream function (cf. Dean and Montagnon [1949]. Michael [1958]) 


$$
\psi=\mathrm{r}^{\lambda}[\mathrm{A} \sin (\lambda \phi)+\mathrm{B} \sin ((\lambda-2) \phi)]
$$

this ansatz incorporates the desired symmetry. At $\phi=\pi$, we must satisfy the conditions of zero shear and normal stress,

$$
u_{y}+v_{x}=0,2 \mu v_{y}-p=0
$$

which can be shown to translate into

$$
\begin{aligned}
& \frac{1}{\mathrm{r}_{2}} \psi_{\phi \phi}+\frac{1}{\mathrm{r}} \psi_{\mathrm{r}}-\psi_{\mathrm{rr}}=0 \\
& \frac{1}{\mathrm{r}^{2}} \psi_{\mathrm{rr} \phi}+\frac{1}{\mathrm{r}^{3}} \psi_{\phi \phi \phi}-\frac{3}{\mathrm{r}^{2}} \psi_{\mathrm{r} \phi}+\frac{4}{\mathrm{r}^{3}} \psi_{\phi}=0
\end{aligned}
$$

With the above ansatz, this leads to

$$
\begin{aligned}
& {[\lambda \mathrm{A}+(\lambda-2) \mathrm{B}] \sin (\lambda \pi)=0,} \\
& (\mathrm{~A}+\mathrm{B}) \cos (\lambda \pi)=0 .
\end{aligned}
$$

Clearly, we have nontrivial solutions for $\lambda=3 / 2$. The linearization of the kinematic free surface condition leads to

$$
\mathrm{Uh}^{\prime}=\mathrm{v}=-\psi_{\mathrm{x}}=\psi_{\mathrm{r}},
$$

and hence

$$
\mathrm{h}=\frac{1}{\mathrm{U}} \mathrm{r}^{\lambda}(\mathrm{A}+\mathrm{B})
$$

Symmetric stream functions (i.e. antisymmetric flows) are given by the ansatz

$$
\psi=\mathrm{r}^{\lambda}[\mathrm{A} \cos (\lambda \phi)+\mathrm{B} \cos ((\lambda-2) \phi)]
$$

In this case, (5) leads to 


$$
\begin{aligned}
& {[\lambda \mathrm{A}+(\lambda-2) \mathrm{B}] \cos (\lambda \pi)=0,} \\
& (\mathrm{~A}+\mathrm{B}) \sin (\lambda \pi)=0 .
\end{aligned}
$$

Again, $\lambda=3 / 2$ is a solution; in place of (8) we get $h=0$.

Hence, if surface tension is neglected, cusped solutions with $h \sim \mathrm{r}^{3 / 2}$ are possible; in actual experiments, we should expect to see a combination of the symmetric and antisymmetric solutions. Now, let us consider what happens if surface tension is included. Then we must change several things. First, we must include a term $\frac{\sigma}{\mu U} \psi_{\text {rrr }}$ on the right hand side of the normal stress condition (5); this term arises if we reexpress $h^{\prime \prime}$ using (7). This changes the second part of equation (6) to

$$
(\mathrm{A}+\mathrm{B})\left[2 \cos (\lambda \pi)-\frac{1}{\mathrm{Ca}} \sin (\lambda \pi)\right]=0 \text {. }
$$

Hence $\lambda$ becomes the root of $\tan (\lambda \pi)=2 \mathrm{Ca}$, which approaches $3 / 2$ as $\mathrm{Ca} \rightarrow \infty$. (In the antisymmetric case, we still find $\lambda=3 / 2$ and $h=0$.) Second, the solution (1) cannot be valid all the way up to the cusp. This is because at the cusp the angle of the interface has a jump of $2 \pi$, leading to a delta function singularity in the traction. This point force must be balanced by a singularity in the flow. This consideration led to Richardson's [1968] solution

$$
\psi=\frac{\sigma}{2 \pi \mu} r \log r \sin \phi
$$

The velocity resulting from (12) has a logarithmic singularity at the cusp; the assumption of small perturbation of uniform flow on which the preceding analysis was based is thus rendered invalid. The velocity gradient resulting from (12) is not square integrable and thus there would be an infinite amount of energy dissipation. This raises questions about the physical realizability of a solution such as (12).

On the other hand, in order for the velocity from (12) to be of the same order as U, we must have logr $2 \pi \mathrm{Ca}$. The critical capillarity numbers reported in Section 2 are in excess of 2; as pointed out, we are slightly underestimating $\mathrm{Ca}_{c}$, so we shall consider 2.5 a more realistic value. 
With $\mathrm{Ca}=2.5$, we conclude that $|\operatorname{logr}|$ must be on the order of 15.7 . If we consider $1 \mathrm{~cm}$ as a macroscopic length scale of observation, we conclude that Richardson's solution does not dominate over the macroscopic (approximately uniform) flow until we reach a length scale of approximately $10^{-7} \mathrm{~cm}$. Such length scales are far beyond the means of optical observation and actually reach the limits of the applicability of continuum mechanics. It thus appears reasonable to presume that the physical difficulties posed by Richardson's solution are resolved at the molecular level. Moreover, we expect that our analysis above, based on perturbing uniform flow and ignoring Richardson's solution, is qualitatively valid in the range of parameters where cusps were observed experimentally.

We conclude this section with some remarks about viscoelastic fluids. Let us first consider a linear viscoelastic fluid; we assume zero surface tension. In this case, the Newtonian velocity field given above actually provides a solution even for the viscoelastic case; the stresses, however, are now given by

$$
\mathbf{T}(\mathrm{x}, \mathrm{y})=\int_{0}^{\infty} \mathrm{G}(\mathrm{s}) \mathbf{D}(\mathrm{x}-\mathrm{Us}, \mathrm{y}) \mathrm{ds},
$$

where $G$ denotes the stress relaxation function and $D$ the symmetric part of the velocity gradient. We note that the stresses given by (13) would be less singular at the cusp than in the Newtonian case. On the other hand, if we use the second order fluid approximation, $T=\mu D+\alpha_{1} U \frac{\partial D}{\partial x}$, where $\mu=\int_{0}^{\infty} \mathrm{G}(\mathrm{s}) \mathrm{ds}, \alpha_{1}=-\int_{0}^{\infty} \mathrm{sG}(\mathrm{s}) \mathrm{ds}$, then the stresses for a given velocity field are more singular than in the Newtonian case. The second order fluid cannot be a valid approximation close to the cusp, but it may be valid some distance away from the cusp.

It appears doubtful whether linear viscoelasticity is very useful in interpreting experiments in non-Newtonian fluids. The extensional behavior of such fluids is highly nonlinear, leading to extensional stresses much larger than those in Newtonian fluids with the same shear viscosity. We note that a non-cusped interface necessarily has a stagnation point, with the associated potential for 
a build-up of elongational stresses. Cusping gets rid of the stagnation point and alleviates some of these elongational stresses. This effect should favor cusping in non-Newtonian fluids. The behavior of non-Newtonian fluids at cusps and in general at corners larger than $180^{\circ}$ is not well understood (see Davies [1988] for some partial results).

We do not understand the mechanism leading to a critical capillary number even in the Newtonian case. For non-Newtonian fluids, more dimensionless quantities can be formed, in addition to the capillary number introduced above. The critical capillary numbers displayed in Table 4 are of the same order of magnitude for non-Newtonian as for Newtonian fluids, but there is a definite trend to lower capillary numbers in the non-Newtonian case. To rectify this, one may think of replacing the Newtonian viscosity in the definition of $\mathrm{Ca}$ by some non-Newtonian viscosity. We note that the extensional viscosity of non-Newtonian fluids generally increases with elongation rate, so that such a procedure would give higher capillary numbers if we think of the flow as being primarily extensional. We give an alternative dimensional analysis based on the second order fluid. For this we estimate elongational stresses as $\left|\alpha_{1}\right| U^{2} / \ell^{2}$, where $\ell$ is a characteristic length, and we estimate stresses resulting from surface tension as $\sigma / \ell$. This leads to the capillary number $\mathrm{Ca}=\left|\alpha_{1}\right| \mathrm{U}^{2} / \sigma \ell$. Next we estimate $\ell$ as $U \tau$, where $\tau$ is a characteristic time scale, so we have $\mathrm{Ca}=\left|\alpha_{1}\right| \mathrm{U} / \sigma \tau$. A possible estimate for $\tau$ is $\left|\alpha_{1}\right| / \mu$; in this case we obtain the same capillary number as in the Newtonian case. It might be argued, however, that shorter relaxation times could be important. Using such shorter relaxation times for $\tau$ would also raise the value of Ca.

\section{Numerical results}

In this section, we present numerical results that show the formation of a cusp in a model problem. The results are computed with the Fluid Dynamics Package (FIDAP) Version 4.0, which uses the finite element method (cf. Engelman and Sani [1986]). 


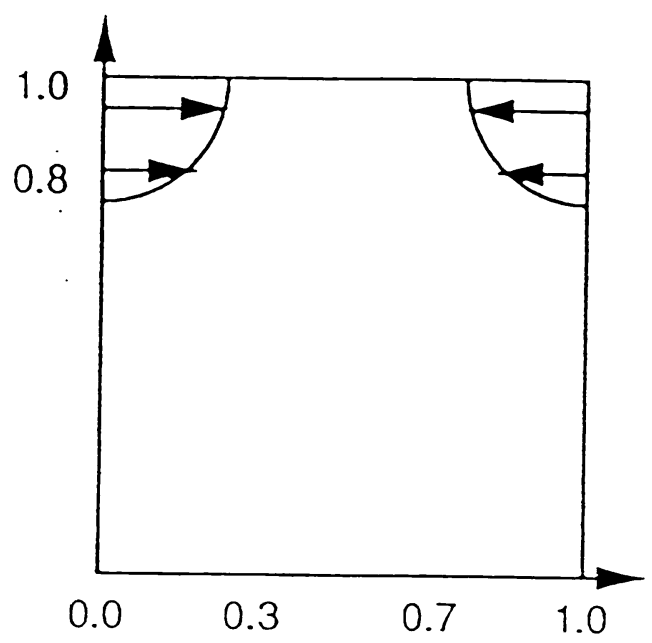

Figure 20. $\quad$ Flow domain for numerical simulations.

The computational domain at time zero is a unit square in the $x-y$ plane, as shown in Figure 20. The top boundary at $y=1,0 \leq x \leq 1$, is a free surface. Along the upper parts of the vertical sides $(v=0,0.8 \leq y \leq 1$ and $x=1,0.8 \leq y \leq 1)$, the boundary condition is zero vertical velocity and the horizontal velocity is half of a parabolic velocity profile. The latter has a maximum value of unity at $y=1$. The boundary along $0 \leq y \leq 0.8$ at $x=0$ and 1 , and along $0 \leq x \leq 0.3$ at $y=0$, and $0.7 \leq x \leq 1.0$ at $y=0$ is a solid wall. Along $0.3 \leq x \leq 0.7$ at $y=0$, the horizontal velocity is zero and the normal traction is 1 , so that there is a negative pressure sucking the fluid out. The viscosity and density of the fluid are prescribed to be unity. The surface tension is varied, as described below.

A mixed velocity pressure formulation is used. Interior elements are nine-node isoparametric quadrilaterals with biquadratic velocity and discontinuous linear pressure functions. Three-node quadratic elements are used on the free surface.

An initial velocity field is computed by solving the Stokes problem with the position of the free surface kept fixed. The transient problem is them computed with the implicit backward Euler integration scheme. At each time-step, the Newton-Raphson scheme is used with a relaxation constant of 0.5 . The initial timestep is 0.01 , and thereafter, a variable time increment is selected automatically by the code. 
We present results for various values of the surface tension. Figures 20 and 21 display the formation of a cusp for zero surface tension. The mesh size is 21 by 17, and the mesh is displayed in each plot. There are 357 nodes, 80 interior elements and 10 elements on the free surface. The times shown on the plots are $0.02,0.03,0.26,0.89,2.11,3.78,5.60$ and 7.45 . The cusp has started to form by time 3.78. The velocity vector plots for times 0.01 and 3.78 are shown in Figure 23 and 24, respectively.

After the formation of the cusp, wiggles appear in the free surface. We believe that this is due to improper handling of the kinematic free surface condition. At a cusp, the kinematic free surface condition must be suspended; fluid particles can move from the free surface into the interior. The code, however, does not know this. The computed results past the development of the cusp can therefore not be trusted.

When surface tension is 0.1 , the cusp does not form. Instead, a steady state is attained, with a smooth curve at the free surface. Figure 25 shows the evolution of the mesh at time 0.02 , $0.03,0.26,0.82,1.89,3.66,6.66,11.57,19.10,28.91,40.31,53.53,69.30$ and 88.80 . By the latter time, a steady state has been reached. The velocity vector plot at time 88.80 is shown in Figure 26. If a much larger amount of surface tension is present (e.g., 1.0), the free surface does not move appreciably from its initial position, and a cusp does not form.

The evolution of the free surface for surface tension 0.05 is shown in Figure 27, where the times are $0.02,0.03,0.26,0.86,2.17,4.27,7.52,12.01,17.58,21.01$ and 27.2. A cusp starts to form by time 12.01. The velocity vector plot for time 21.01 is shown in Figure 28. At later times, a steady state is reached. These computations have been convergence tested on a finer mesh.

The numerical results of this section show the formation of a cusp in slow viscous flow, for values of the surface tension that are in the order-of-magnitude range observed in the experiments described in the earlier sections. 


\section{Conclusions and Discussion}

1. It is easy to create two-dimensional cusped surfaces at liquid-air interfaces.

2. In all cases studied by us, the formation of cusped surfaces between air and liquid occurred for all non-Newtonian liquids and for some Newtonian liquids. Our criterion to judge whether a fluid is non-Newtonian is that a non-Newtonian fluid will climb a rod.

3. The formation of cusps is a critical phenomenon; other things being equal, the interface is rounded for small values of the streaming speed and becomes cusped at a critical speed and beyond. In non-Newtonian fluids this critical speed is very distinct; in Newtonian fluids it is less distinct and the change from a rounded to a pointed interface appears to be gradual.

4. Cusping is unusual at the interface of two liquids. Higher viscosity liquids in water form rollers, which are replaced by fingering flows when the viscosity of the liquid on the cylinder is too low to support a roller.

5. SAE 30 motor oil and water support a cusped interface with periodic scallops along the cusp which form sites for the fingering of water into oil. The motor oil is Newtonian and will not cusp in air.

6. We have given a local analysis of the Stokes equations near a cusp. For zero surface tension, this local analysis yields an interface of the form $y^{2}=-c x^{3}$. A small surface tension would change the exponents and, more seriously, it leads to a point force at the cusp which has to be balanced by a singularity of the flow. This leads to Richardson's solution, which has an infinite backward velocity at the cusp and an infinite rate of energy dissipation. However, we estimate that for the situations on which cusps are actually observed, Richardson's solution would not become dominant until molecular length scales are reached. At such length scales, one would have to modify the physics. 
7. Linear viscoelasticity would predict stresses at a cusp which are less singular than in the Newtonian case. However, it seems likely that nonlinear extensional behavior of nonNewtonian fluids is quite important. Experimentally, non-Newtonian fluids form cusps at lower speeds than Newtonian fluids. A qualitative explanation may be that cusping avoids the stagnation point inevitably present on a smooth interface and thus relieves extensional stresses.

8. Numerical simulations show results which are in qualitative agreement with the experiments; cusps form for low values of surface tension, while a steady smooth interface is reached at higher values.

\section{Acknowledgements}

The work of D.D. Joseph and J. Nelson was supported by the National Science Foundation, the Army Research Office, Mathematics and the Department of Energy. M. and Y. Renardy were supported by the National Science Foundation under Grants DMS-8796241 and DMS-8902166, respectively.

\section{REFERENCES}

Acrivos, A. \& Lo, T.S. 1978 Deformation and breakup of a single slender drop in an extensional flow. J. Fluid Mech. 86, 641-672.

Astarita, G. \& Apuzzo, G. 1965 Motion of gas bubbles in non-Newtonian liquids. A. I. Ch. E. Jl. 11, 815-820.

Barnett, S.M., Humphrey, A.E. \& Litt, M. 1966 Bubble motion and mass transfer in nonNewtonian fluids. A.I. Ch. E. Jl. 12, 253-259.

Buckmaster, J.D. 1972 Pointed bubbles in slow viscous flow. J. Fluid Mech. 55, 385-400. 
Joseph, D.D., Nguyen, K. \& Beavers, G.S. 1984 Non-uniqueness and stability of the configuration of flow of immiscible fluids with different viscosities. J. Fluid Mech. 141, 319-345.

Leal, L.G., Skoog, J. \& Acrivos, A. 1971 On the motion of gas bubbles in a viscoelastic liquid. Can. J. Chem. Engng. 49, 569-575.

Mhatre, M.V. \& Kintner, R.C. 1959 Fall of liquids drops through pseudoplastic liquids. Ind. Engng. Chem. 51, 865-867.

Michael, H.D. 1958 The separation of a viscous liquid at a straight edge. Mathematika 5, 82-84.

Philoppoff, W. 1937 The viscosity characteristics of rubber solutions.. Rubb. Chem. Technol. 10, 76-104.

Rallison, J.M. \& Acrivos, A. 1978 A numerical study of the deformation and burst of a viscous drop in an extensional flow. J. Fluid Mech. 89, 191-200.

Rayleigh, Lord 1890 On the theory of surface forces. Phil. Mag., 5th ser. 34, 285-298 and 456475.

Richardson, S. 1968 Two dimensional bubbles in slow flow. J. Fluid Mech. 33, 475-493.

Rumscheidt, F.D. \& Mason, S.G. 1961 Particle motion in sheared suspensions. XII. Deformation and burst of fluid drops in shear and hyperbolic flow. J. Colloid. Sci.16, 238-261.

Sherwood, J.D. 1981 Spindle shaped drops in a viscous extensional flow. Math. Proc. Camb. Phil. Soc. 90, 529-536.

Taylor, G.I. 1934 The formation of emulsions in definable fields of flow. Proc. Roy. Soc. A 146, 501-523. 
Taylor, G.I. 1964 Conical free surfaces and fluid interfaces. Proc. 11th Int. Cong. Appl. Mech., Munich.

Warshay, M.E., Bougsz, E., Johnson, M. \& Kintner, R.C. 1959 Ultimate velocity of drops in stationary liquid media. Can. J. Chem. Engng. 37, 29-36.

Zana, E. \& Leal, L.G. 1978 The dynamics and dissolution of gas bubbles in a viscoelastic fluid. Int. J. Multiphase Flow 4, 237-262.

\section{LIST OF FIGURES}

Figure 3. Cylinder coated with 12,500 cs silicone oil cusps in air. a) liquid is dragged down between the cylinders, b) liquid is dragged up between cylinders.

Figure 4. Figure 4. Cusp point on the $12,500 \mathrm{cs}$ silicone oil as seen through a Nikon microscope.

Figure 5. Cylinder coated with STP (polyisobutylene in petroleum oil) cusps in air. a) liquid is dragged down between the cylinders, b) liquid is dragged up between cylinders.

Figure 6. Cylinder coated with TLA 510 cusps in air. a) liquid is dragged down between the cylinders, b) liquid is dragged up between the cylinders.

Figure 7. Cylinder coated with M1 (polyisobutylene in kerosene and polybutene) cusps in air. a) 19 RPM, b) 75 RPM.

Figure 8. a) SAE 30 motor oil does not cusp in air, b) a cusped oil-water interface with scallops forming between SAE 30 and water.

Figure 9. The critical speed for SAE 30 motor oil and water is approximately 51 RPM. a) 75 RPM, b) 51 RPM, c) 43 RPM, d) 27 RPM.

Figure 10. SAE 30 motor oil and water at 75 RPM. The cusp is not two dimensional but is scalloped. The scallops are sites where water bubbles through into the motor oil.

Figure 11. Low molecular weight silicone oils do not cusp. a) $500 \mathrm{cs}$ is marginal, but a Nikon microscope shows it does cusp. b) 200 cs silicone oil does not cusp.

Figure 12. Glycerine does not cusp in air. 
Figure 13. Critical speed for cusping of M1 in air is approximately 2.4 RPM. a) 2 RPM, b) 75 RPM (cf. Figure 7).

Figure 14. Critical speed for cusping of STP is approximately 2.9 RPM. a) 2 RPM, the bump on the rounded end is an air bubble emerging through the interface, b) 24 RPM.

Figure 15. Critical speed for cusping of $2 \%$ aqueous polyox in air is approximately .75 RPM. a) 7 RPM, b) 7 RPM, c) 27 RPM.

Figure 16. Critical speed for cusping of $1 \%$ aqueous polyox in air is 51.7 RPM. a) 27 RPM, b) 67 RPM, c) 75 RPM, d) 75 RPM.

Figure 17. The critical speed for $500 \mathrm{cs}$. silicone oil is approximately 75 RPM. a) 44 RPM, b) 59 RPM, c) 65 RPM, d) 70 RPM, e) 75 RPM.

Figure 18. The critical rotation speed for castor oil is approximately 113 RPM. a) 122 RPM, b) 113 RPM, c) 85 RPM, d) 67 RPM.

Figure 21. Surface tension zero, times $0.02,0.03,0.26,0.89$.

Figure 22. Surface tension zero, times $2.11,3.78,5.60,7.45$.

Figure 23. Surface tension zero, time 0.01 .

Figure 24. Surface tension zero, time 3.78.

Figure 25(a). Surface tension 0.1 , times $0.02,0.03,0.26,0.82$.

Figure 25(b). Surface tension 0.1, times 1.89, 3.66, 6.66, 11.57.

Figure 25(c). Surface tension 0.1, times 19.10, 28.91, 40.31, 53.53.

Figure 25(d). Surface tension 0.1, times 69.30, 88.80.

Figure 26. Surface tension 0.1 , time 88.80 .

Figure 27(a). Surface tension 0.05 , times $0.02,0.03,0.26,0.86$.

Figure 27(b). Surface tension 0.05 , times $2.17,4.27,7.52,12.01$.

Figure 27(c). Surface tension 0.05, times 17.58, 21.01, 27.2.

Figure 28. Surface tension 0.05 , time 21.01. 


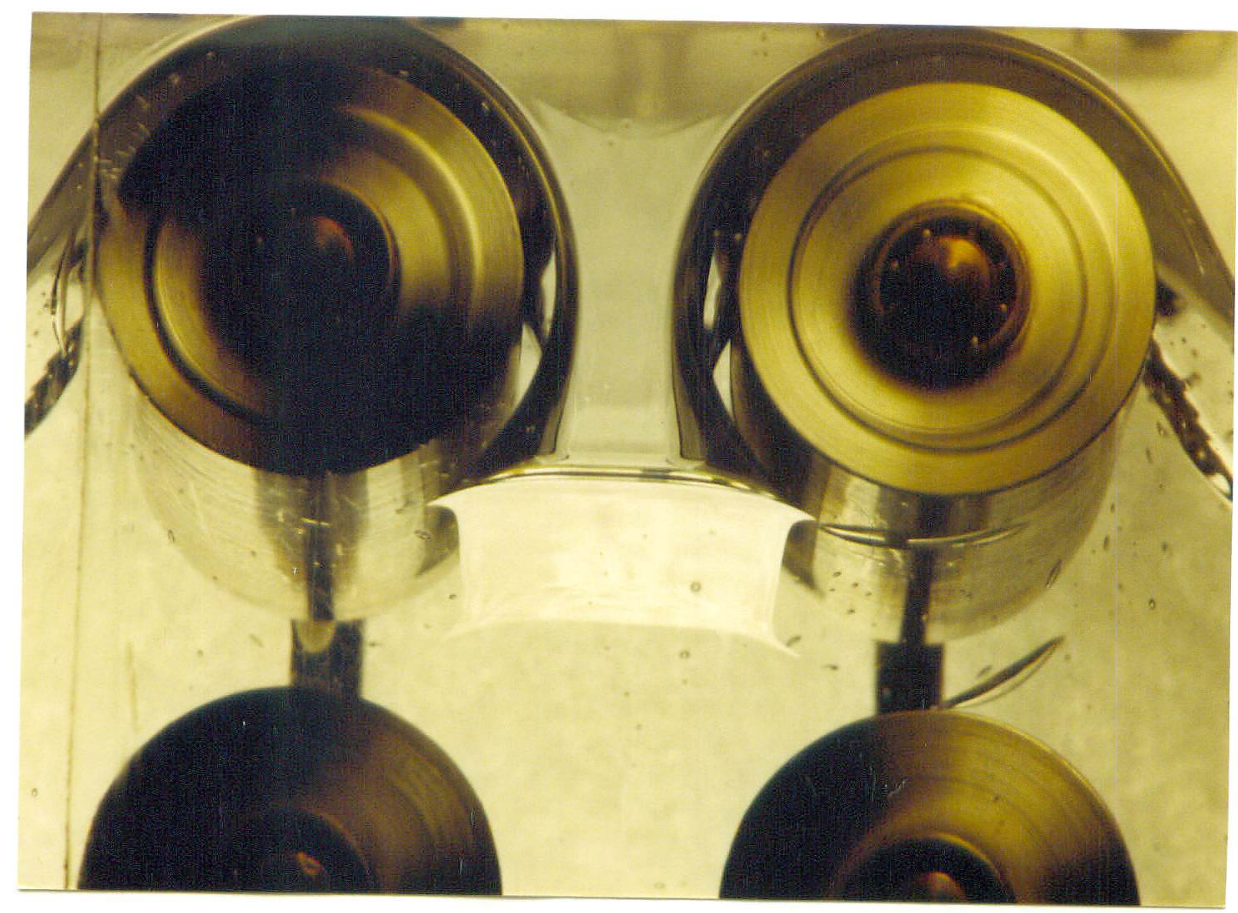

(a)

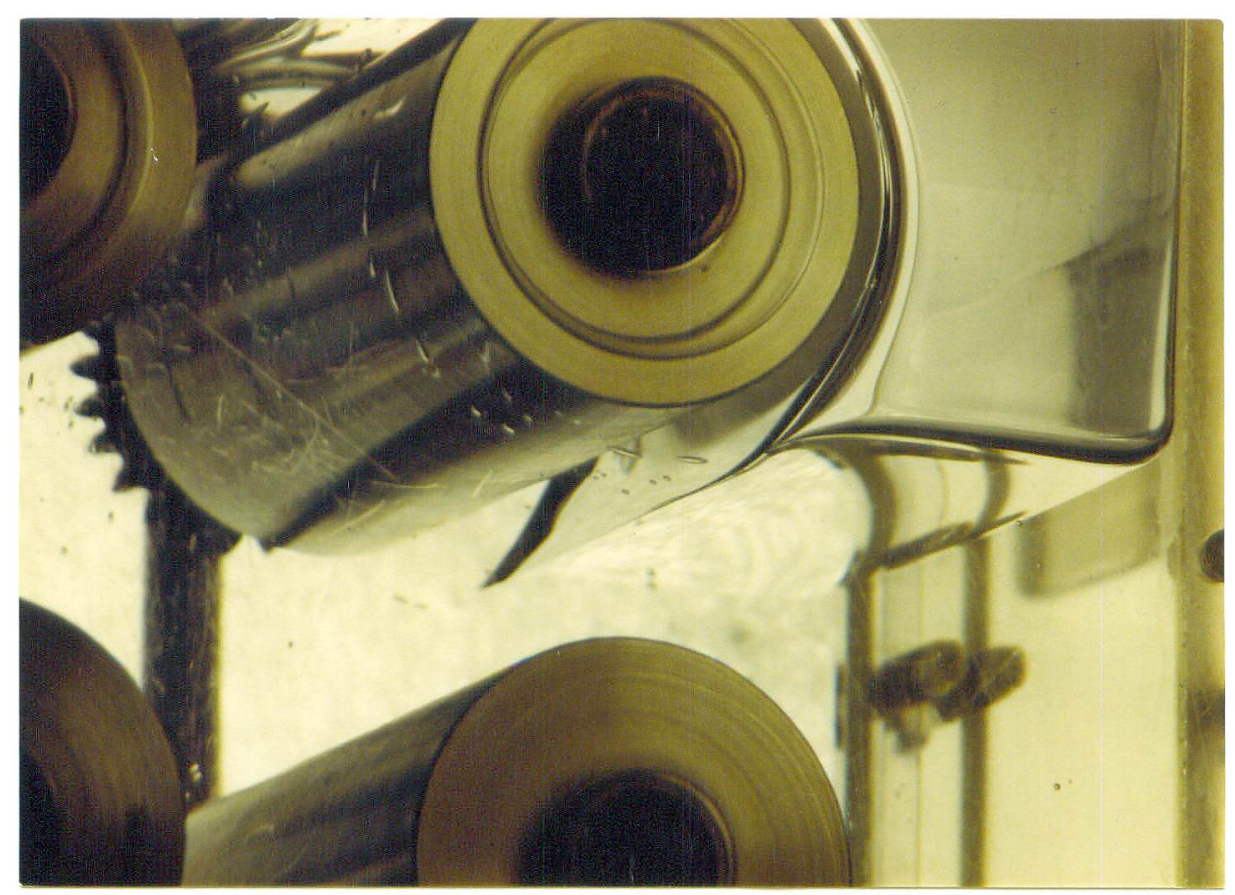

(b)

Figure 3. Cylinder coated with $12,500 \mathrm{cs}$ silicone oil cusps in air. a) liquid is dragged down between the cylinders, b) liquid is dragged up between cylinders. 


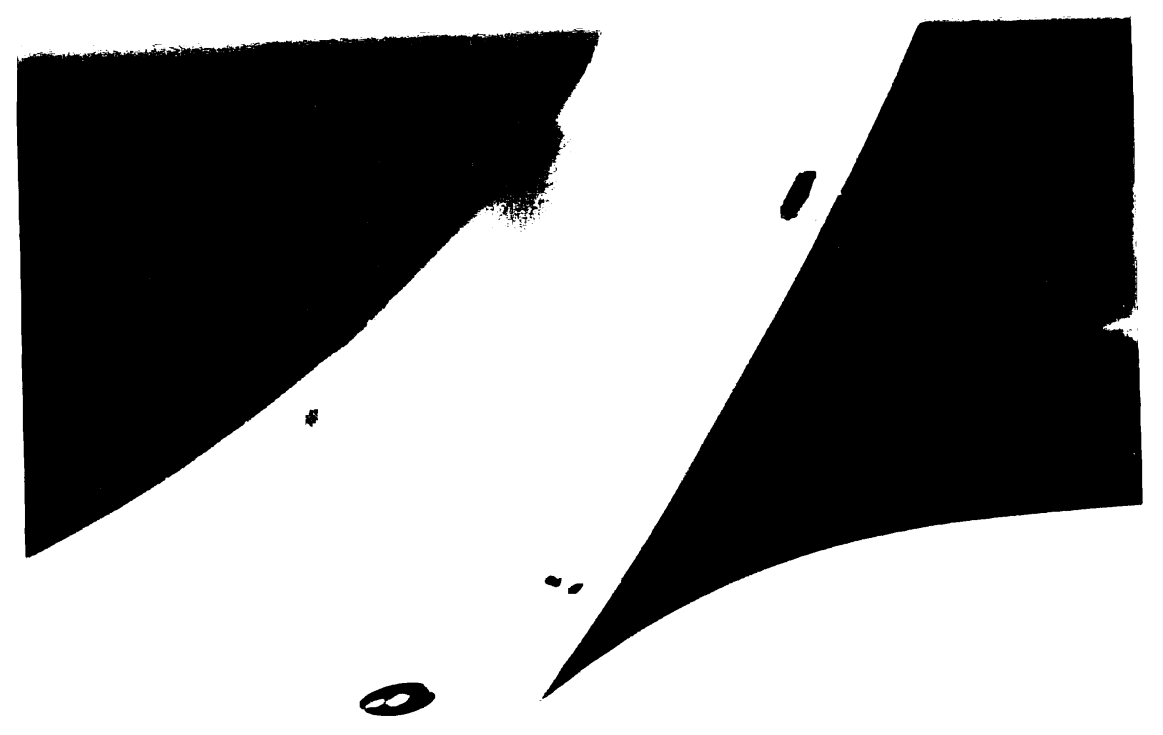

Figure 4. Figure 4. Cusp point on the $12,500 \mathrm{cs}$ silicone oil as seen through a Nikon microscope. 


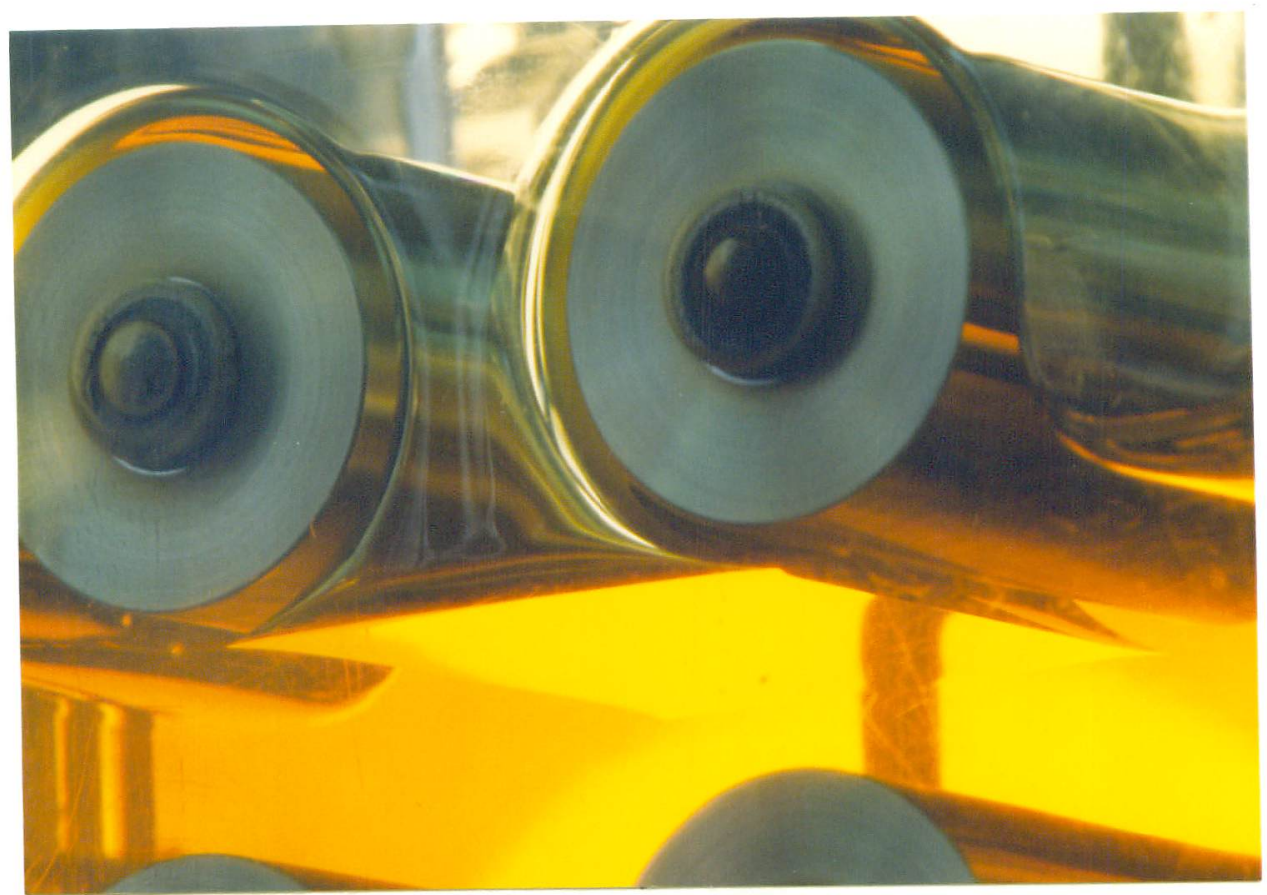

(a)

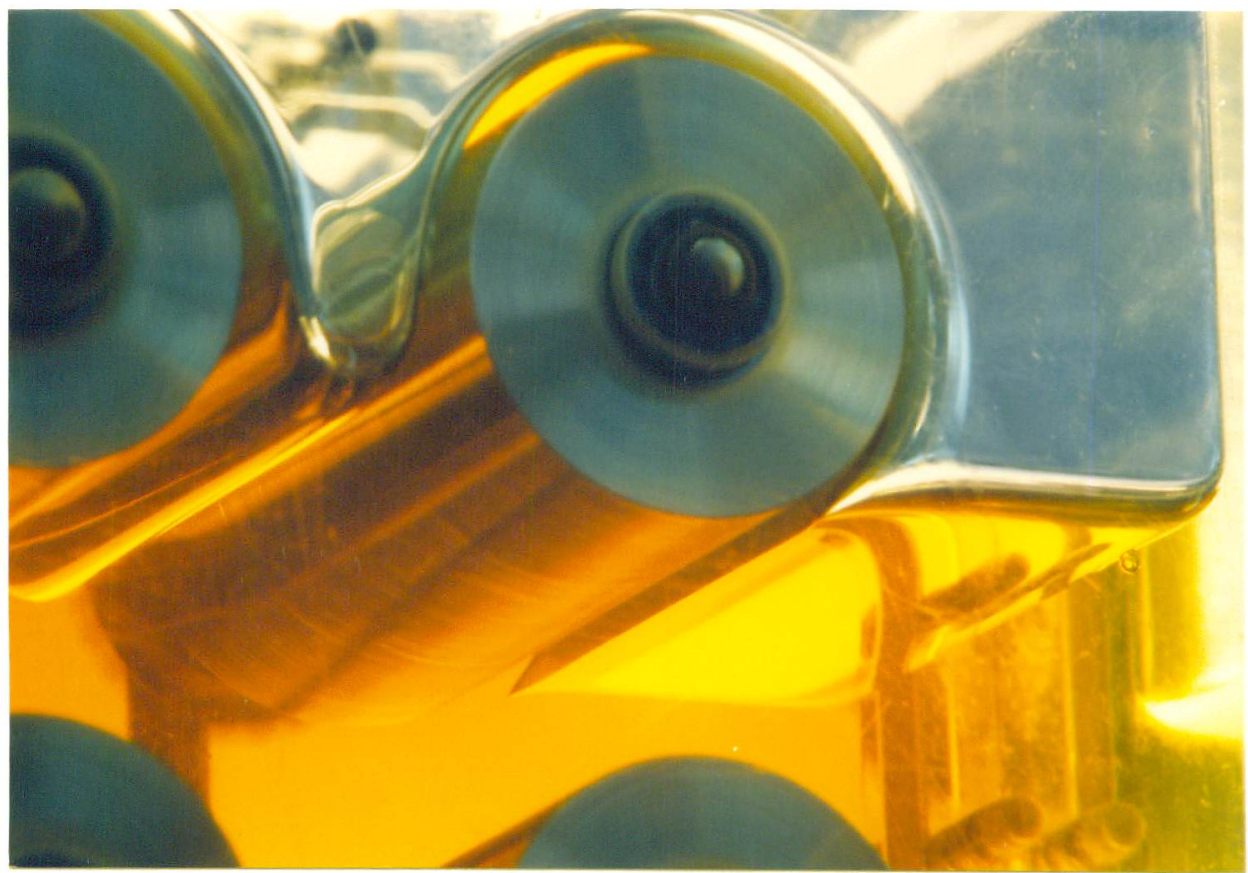

(b)

Figure 5. Cylinder coated with STP (polyisobutylene in petroleum oil) cusps in air. a) liquid is dragged down between the cylinders, b) liquid is dragged up between cylinders. 


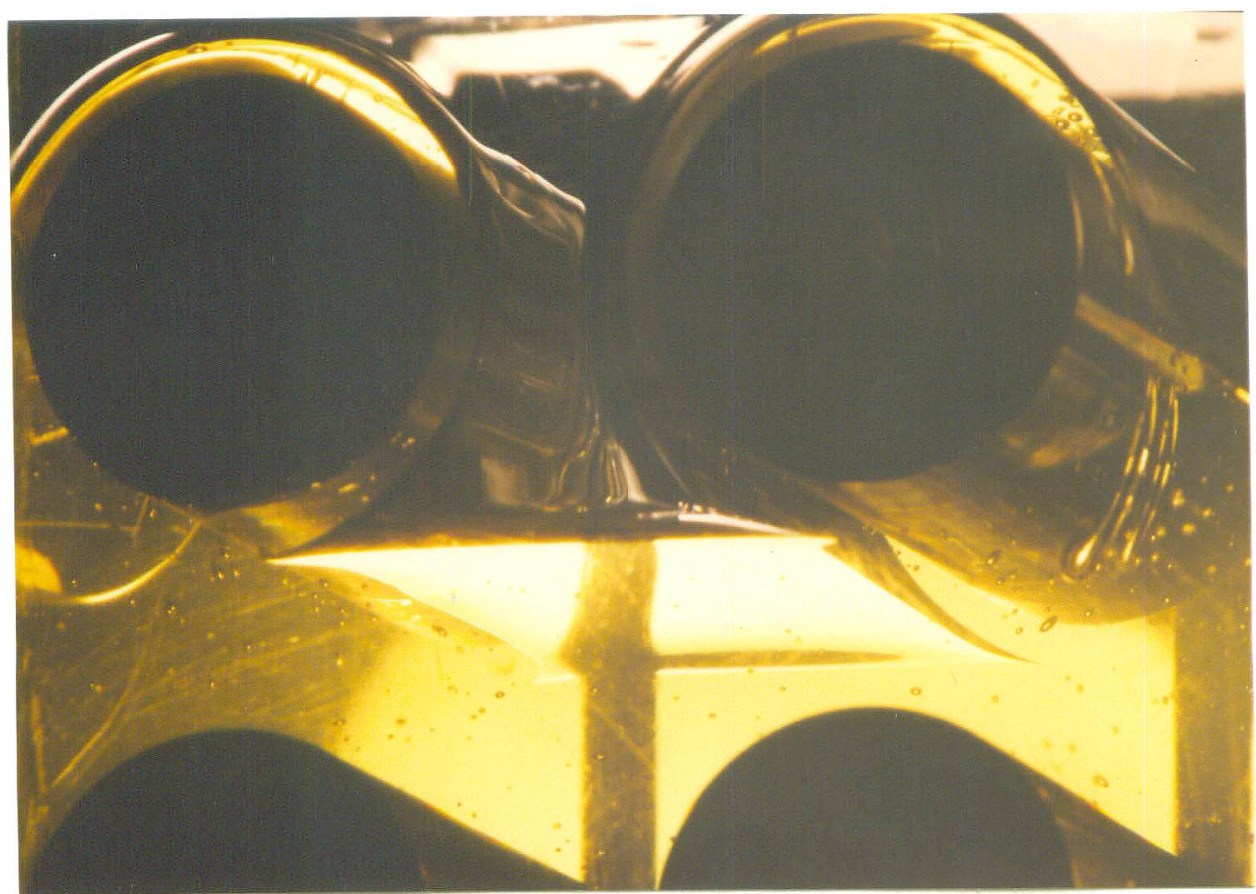

(a)

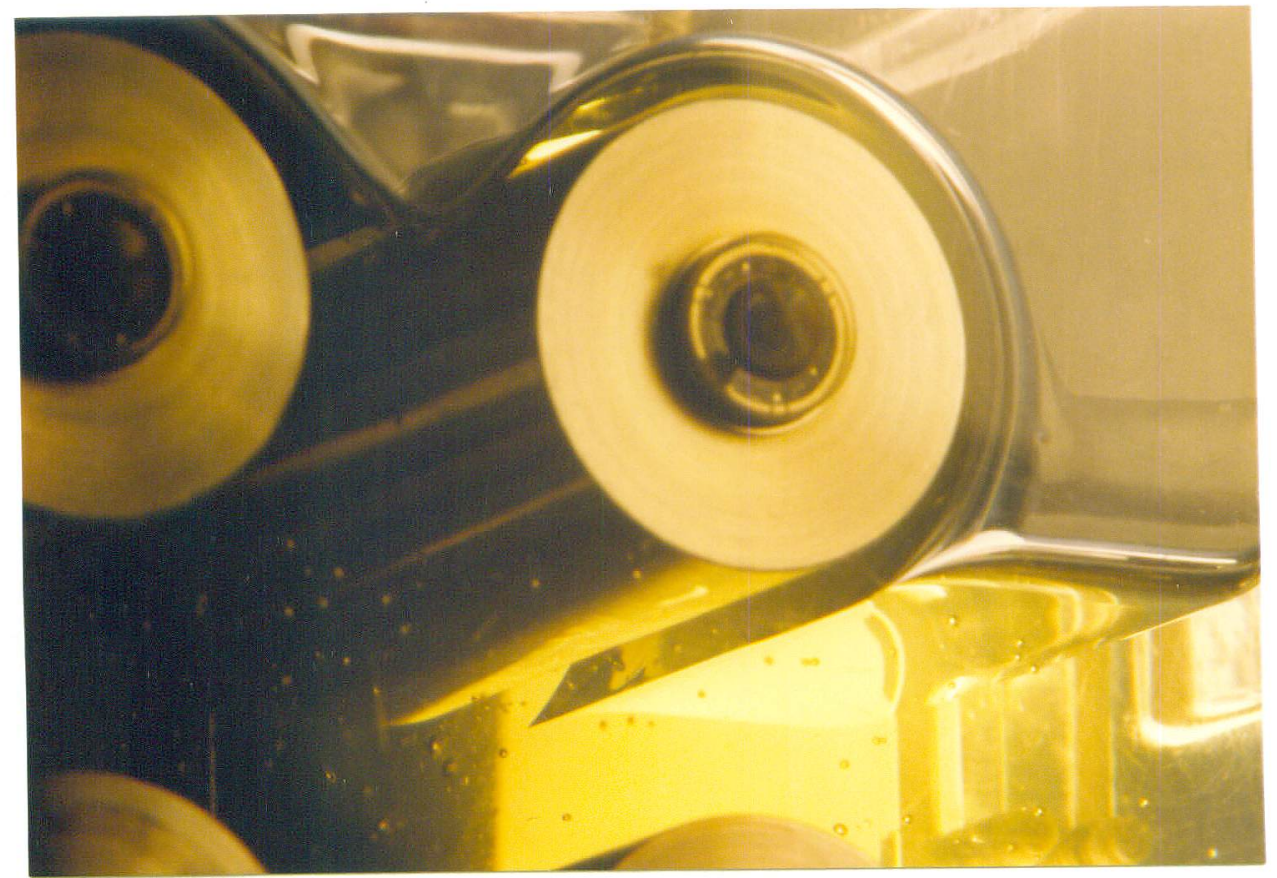

(b)

Figure 6. Cylinder coated with TLA 510 cusps in air. a) liquid is dragged down between the cylinders, b) liquid is dragged up between the cylinders. 


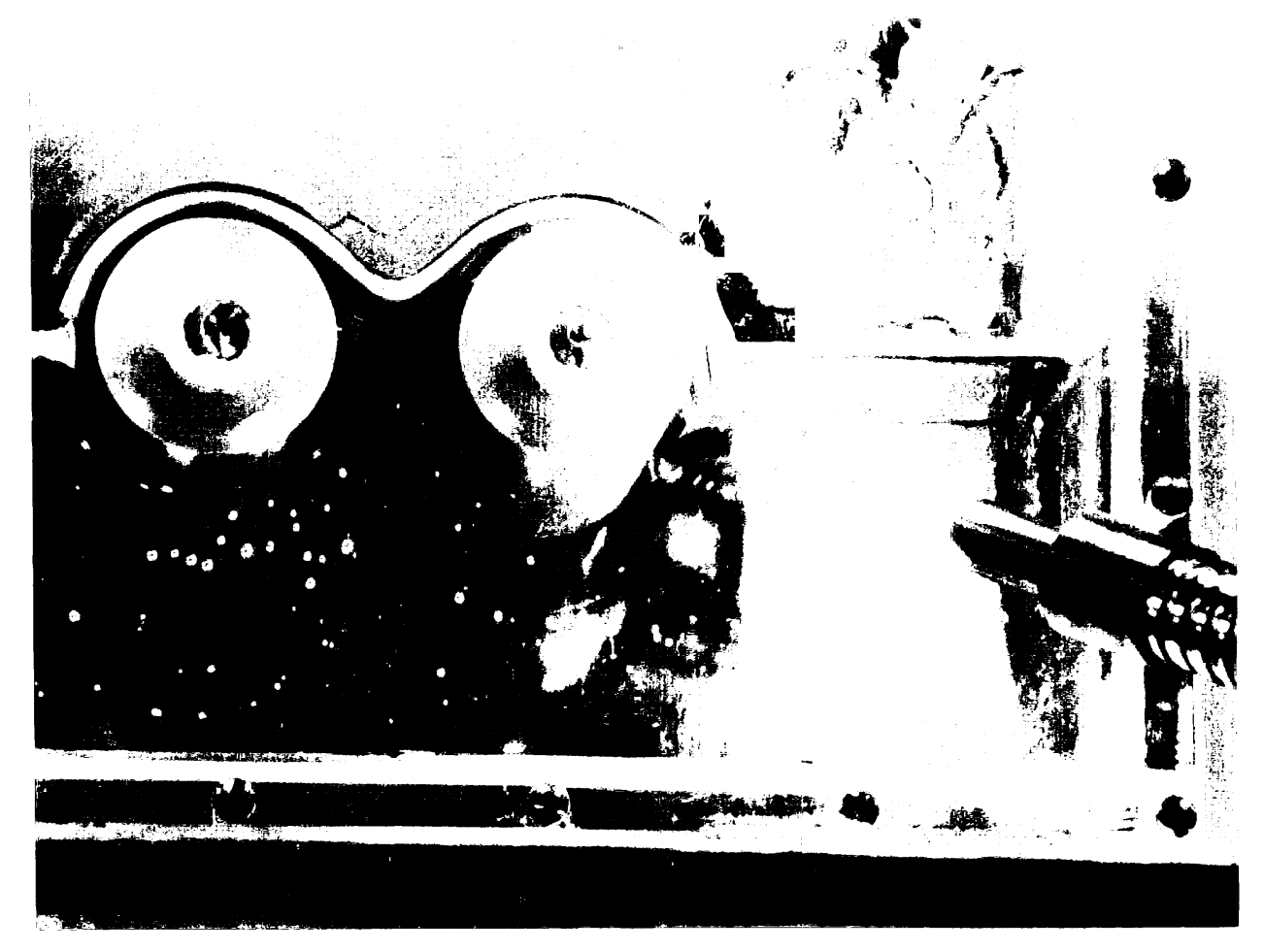

(a)

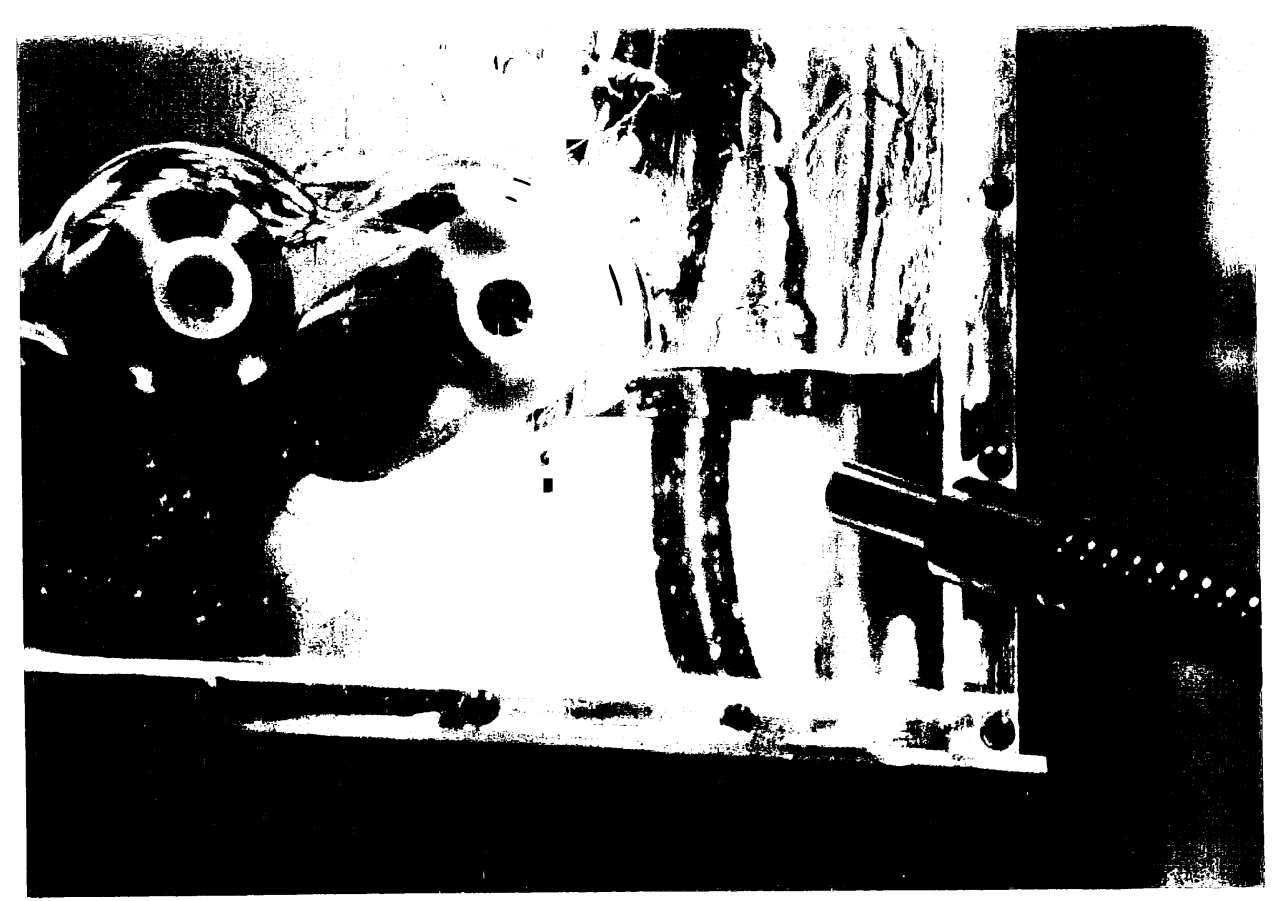

(b)

Figure 7. Cylinder coated with M1 (polyisobutylene in kerosene and polybutene) cusps in air. a) 19 RPM, b) 75 RPM. 


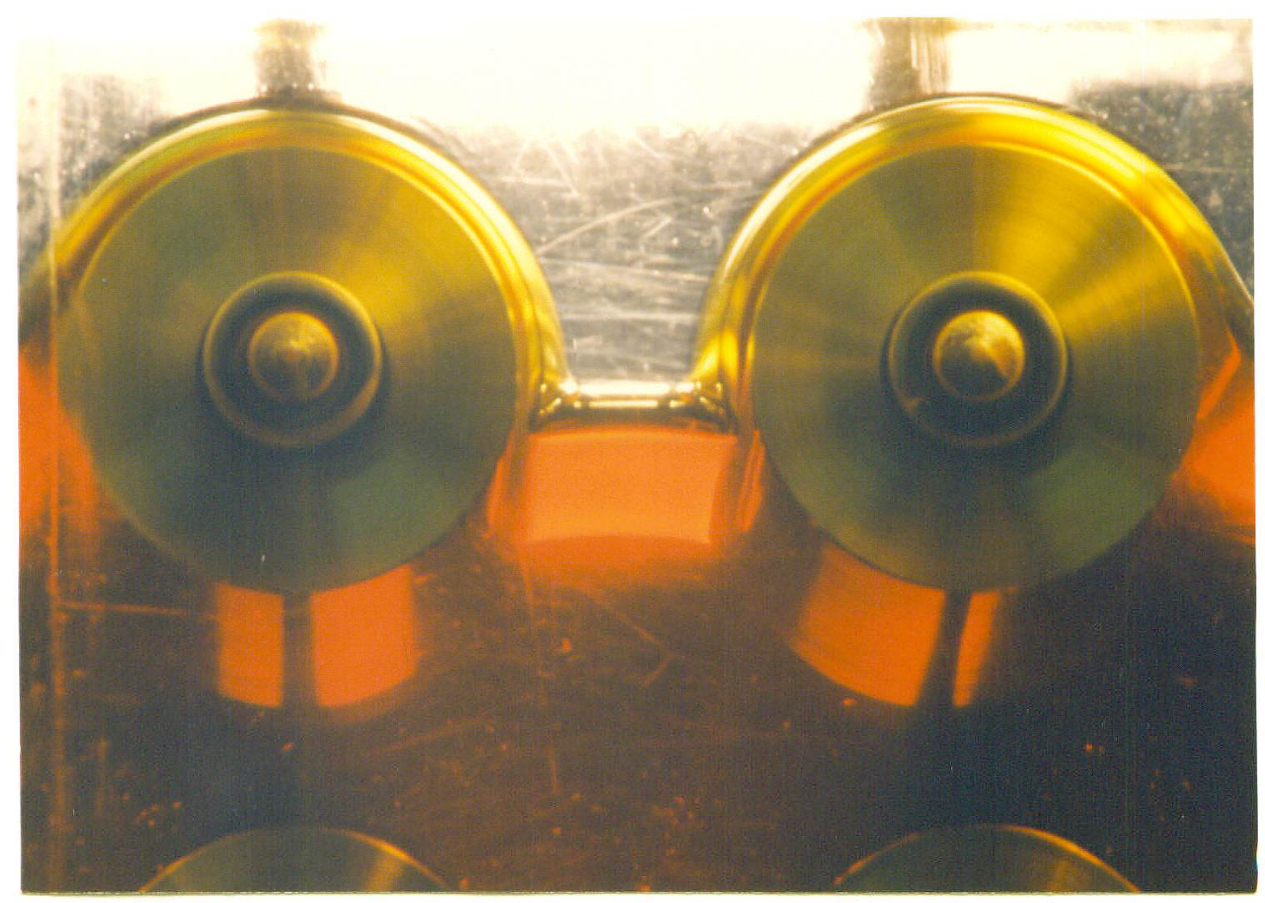

(a)

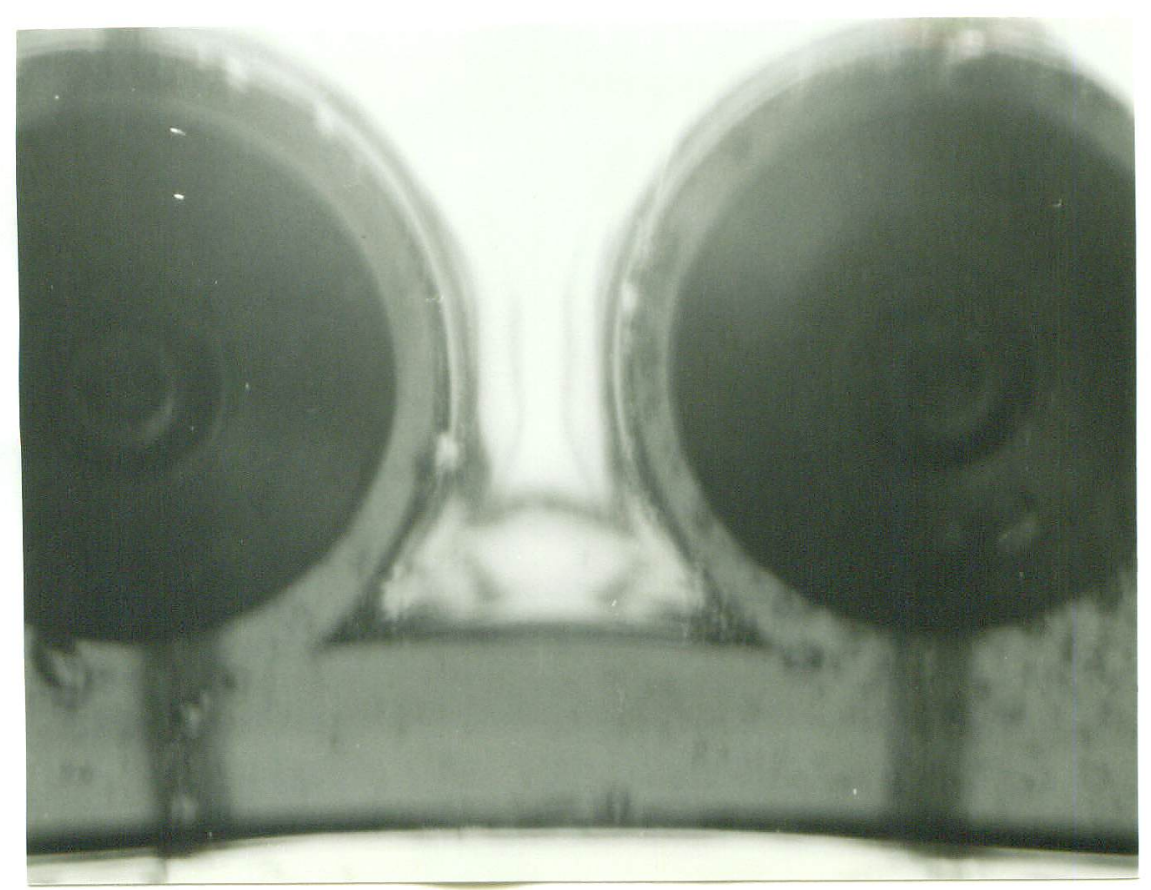

(b)

Figure 8. a) SAE 30 motor oil does not cusp in air, b) a cusped oil-water interface with scallops forms between SAE 30 and water. 


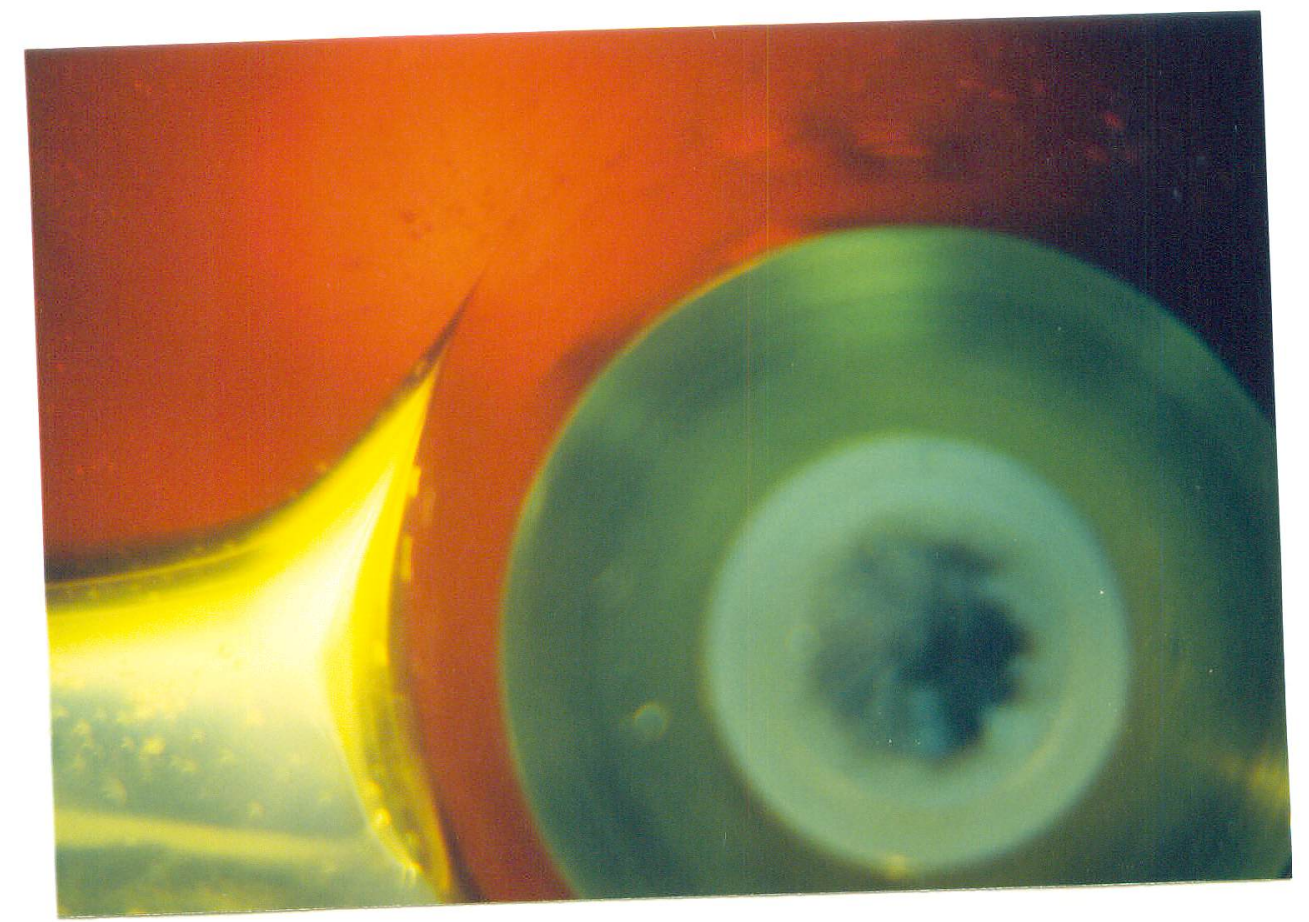

(a)

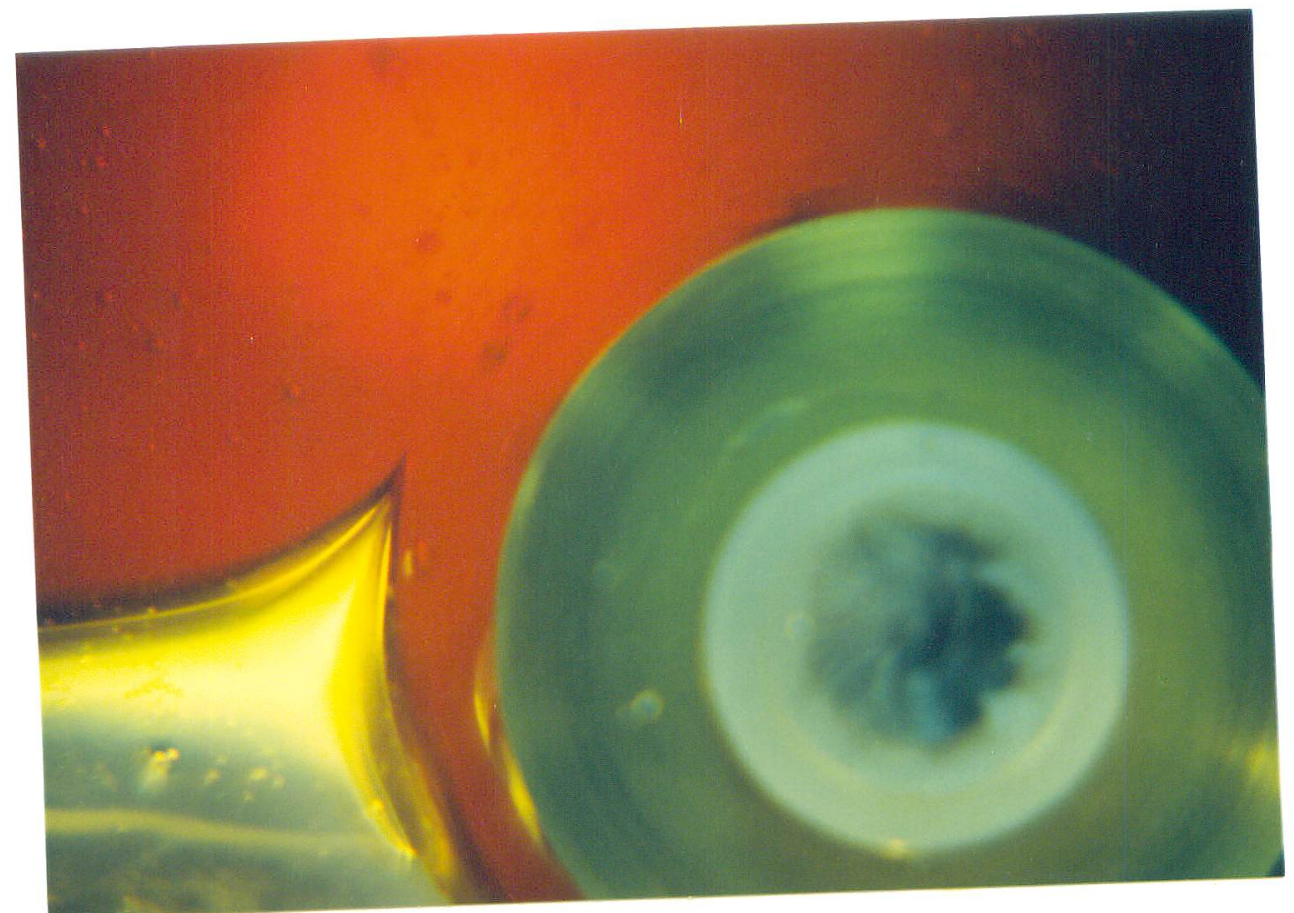

(b) 


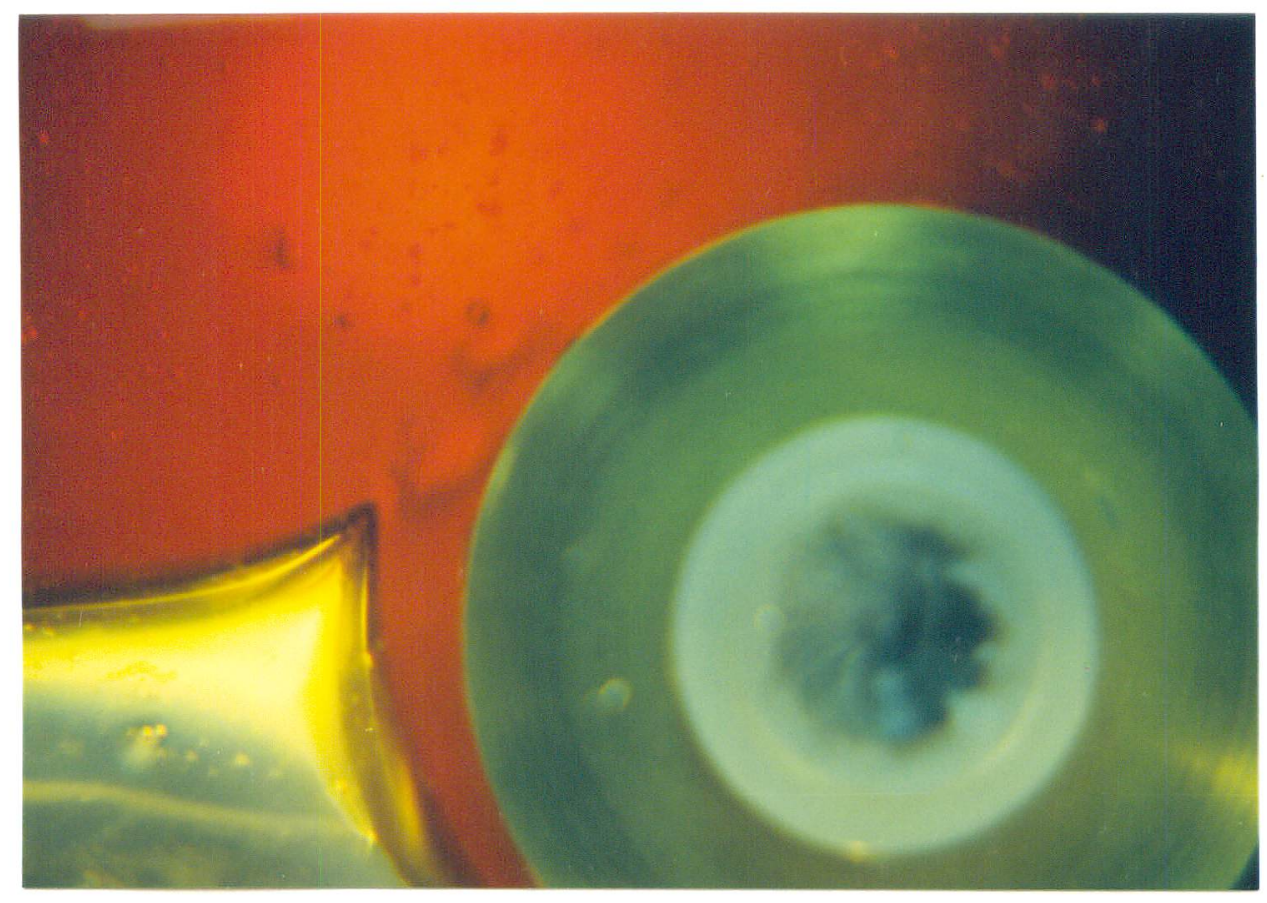

(c)

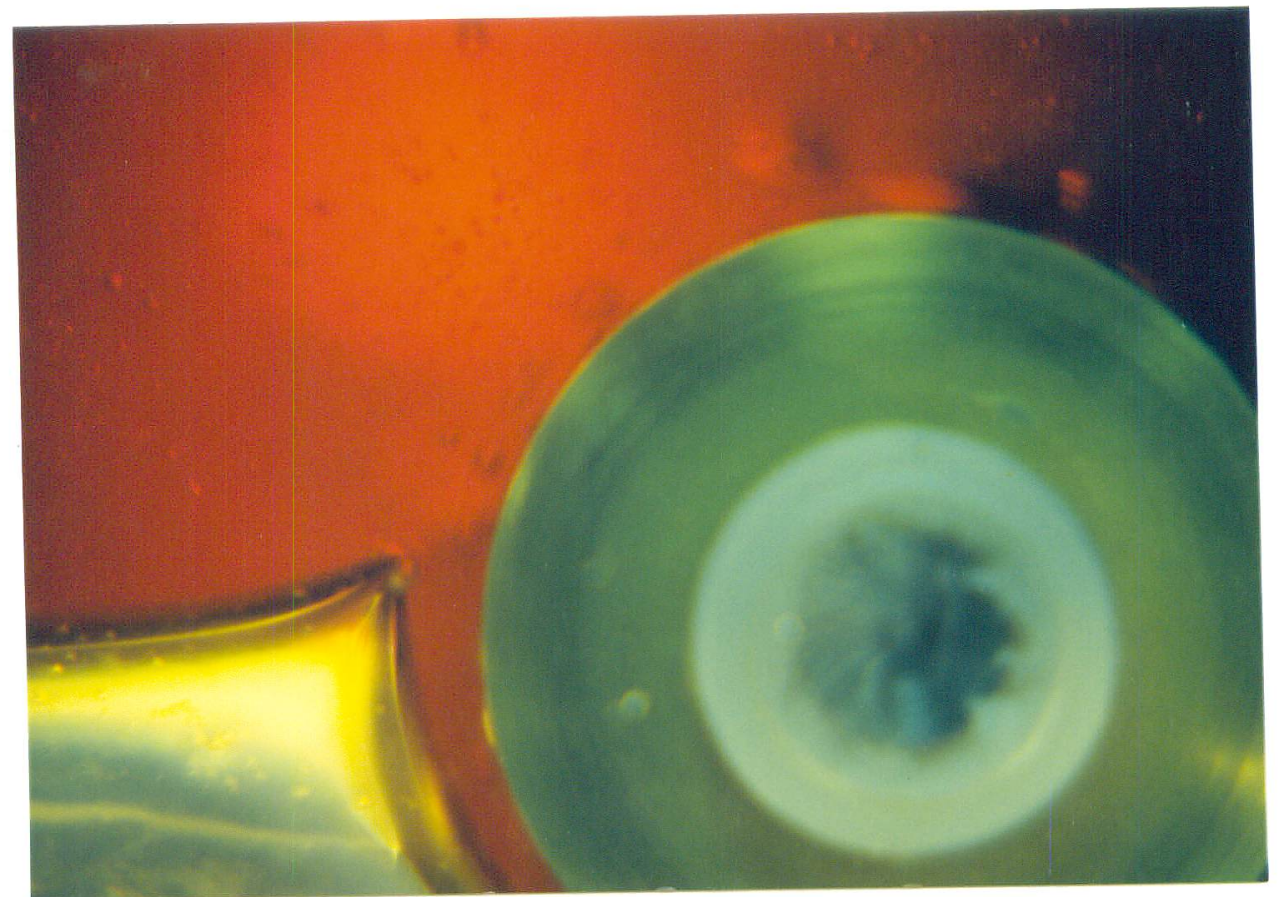

(d)

Figure 9. The critical speed for SAE 30 motor oil and water is approximately 51 RPM. a) 75 RPM, b) 51 RPM, c) 43 RPM, d) 27 RPM. 


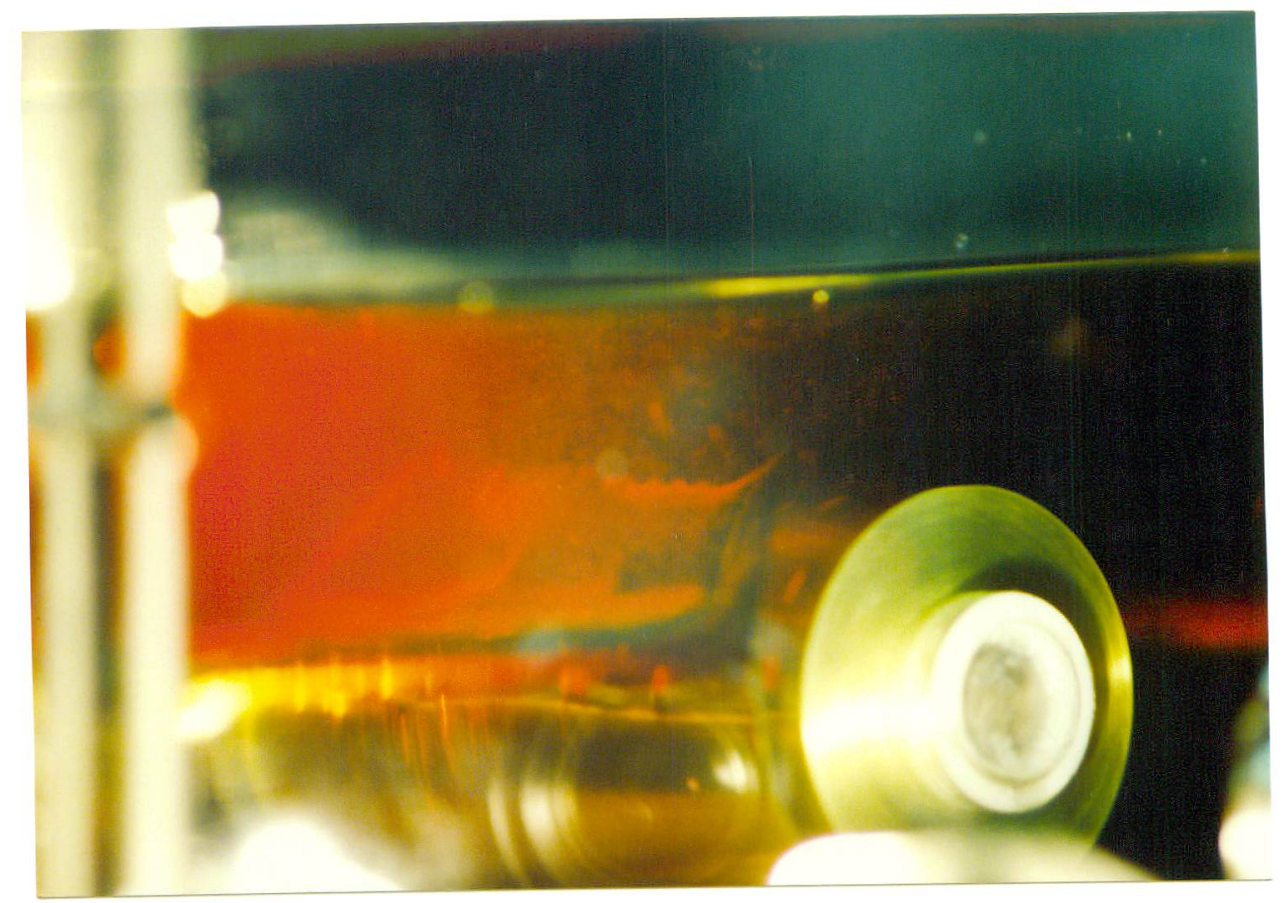

Figure 10. SAE 30 motor oil and water at 75 RPM. The cusp is not two dimensional but is scalloped. The scallops are sites where water bubbles through into the motor oil. 


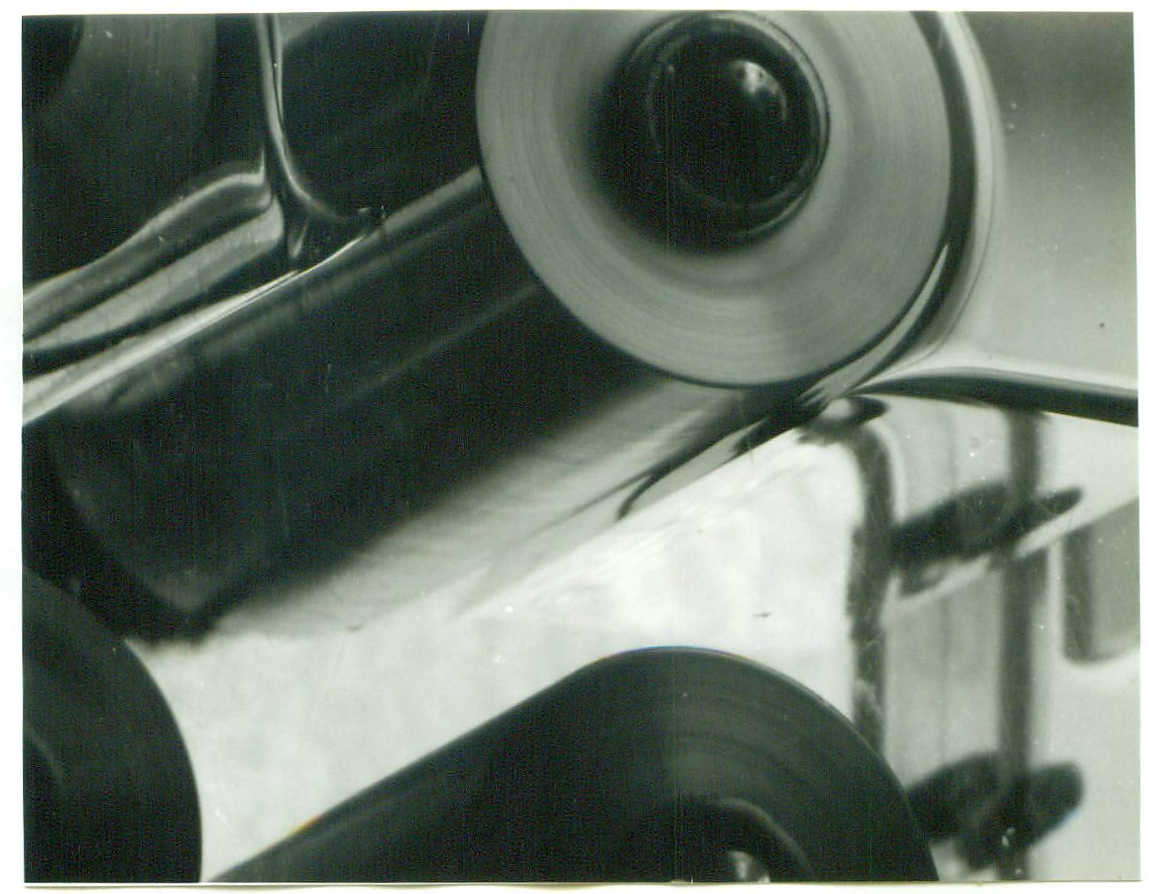

(a)

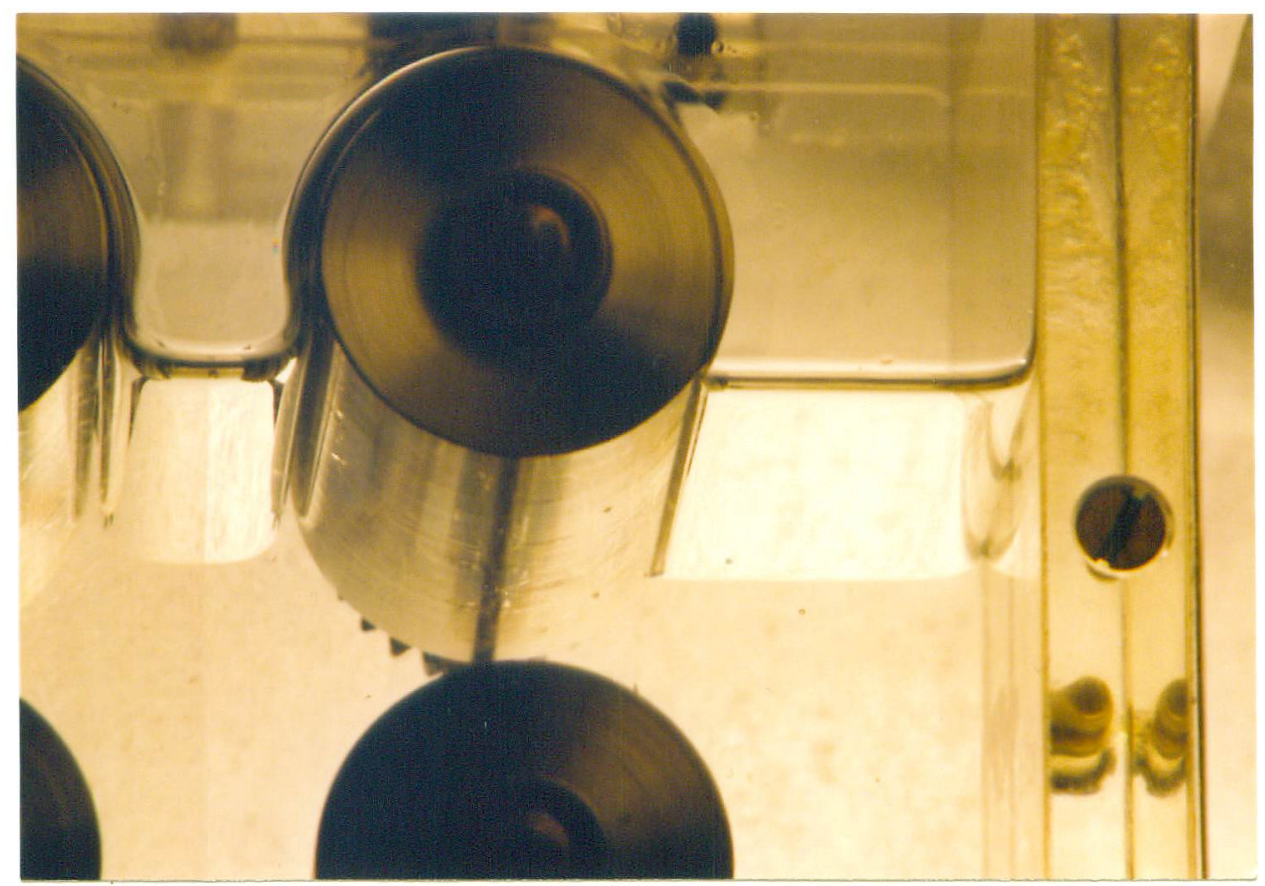

(b)

Figure 11. Low molecular weight silicone oils do not cusp. a) $500 \mathrm{cs}$ is marginal, but a Nikon microscope shows it does cusp. b) 200 cs silicone oil does not cusp. 


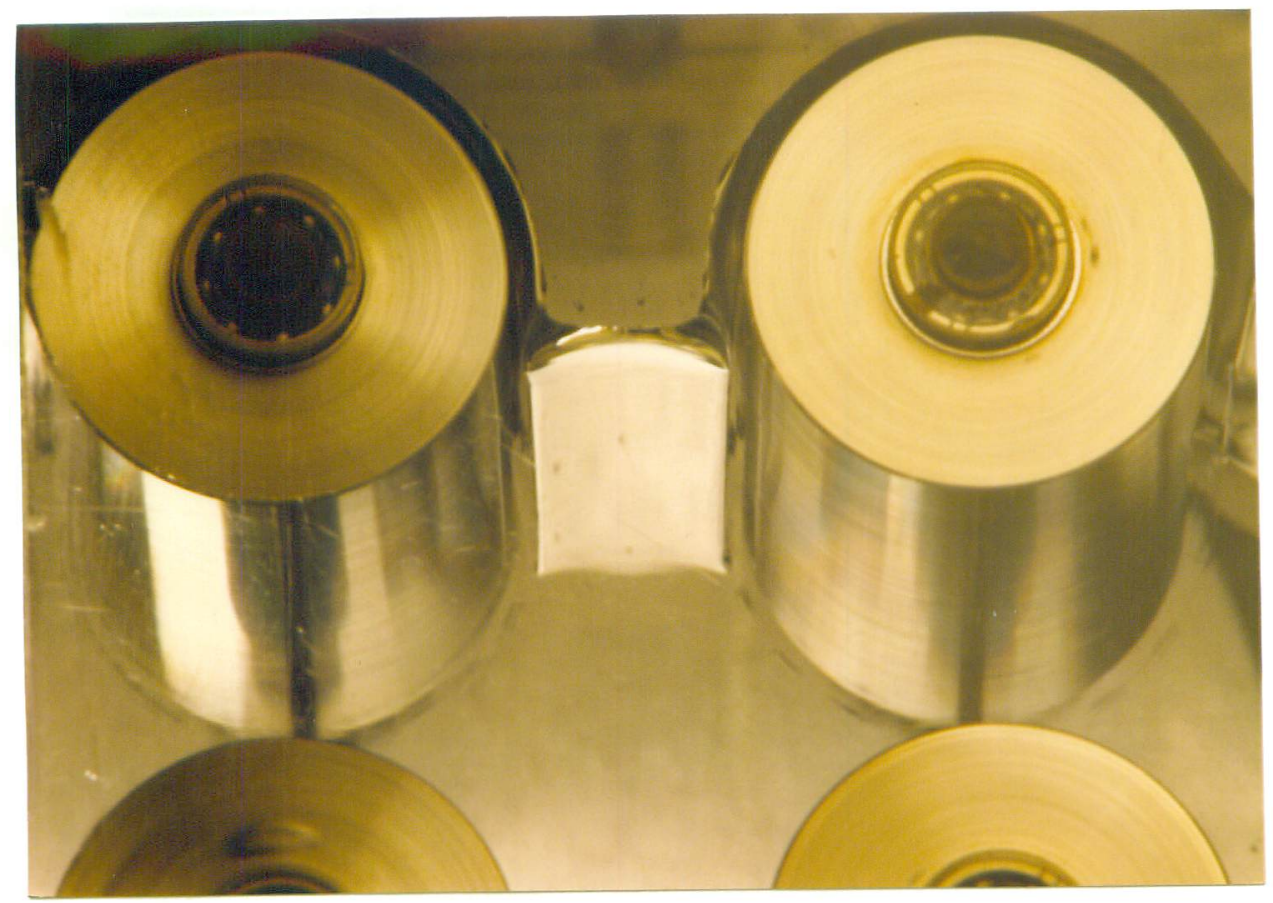

Figure 12. Glycerine does not cusp in air. 


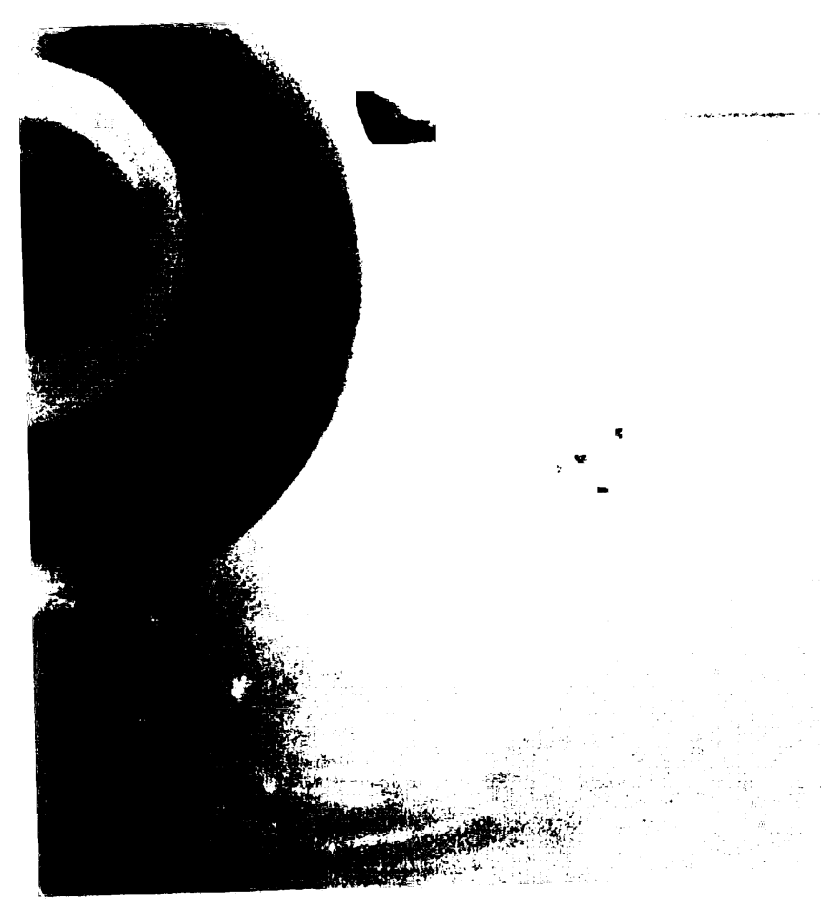

(a)

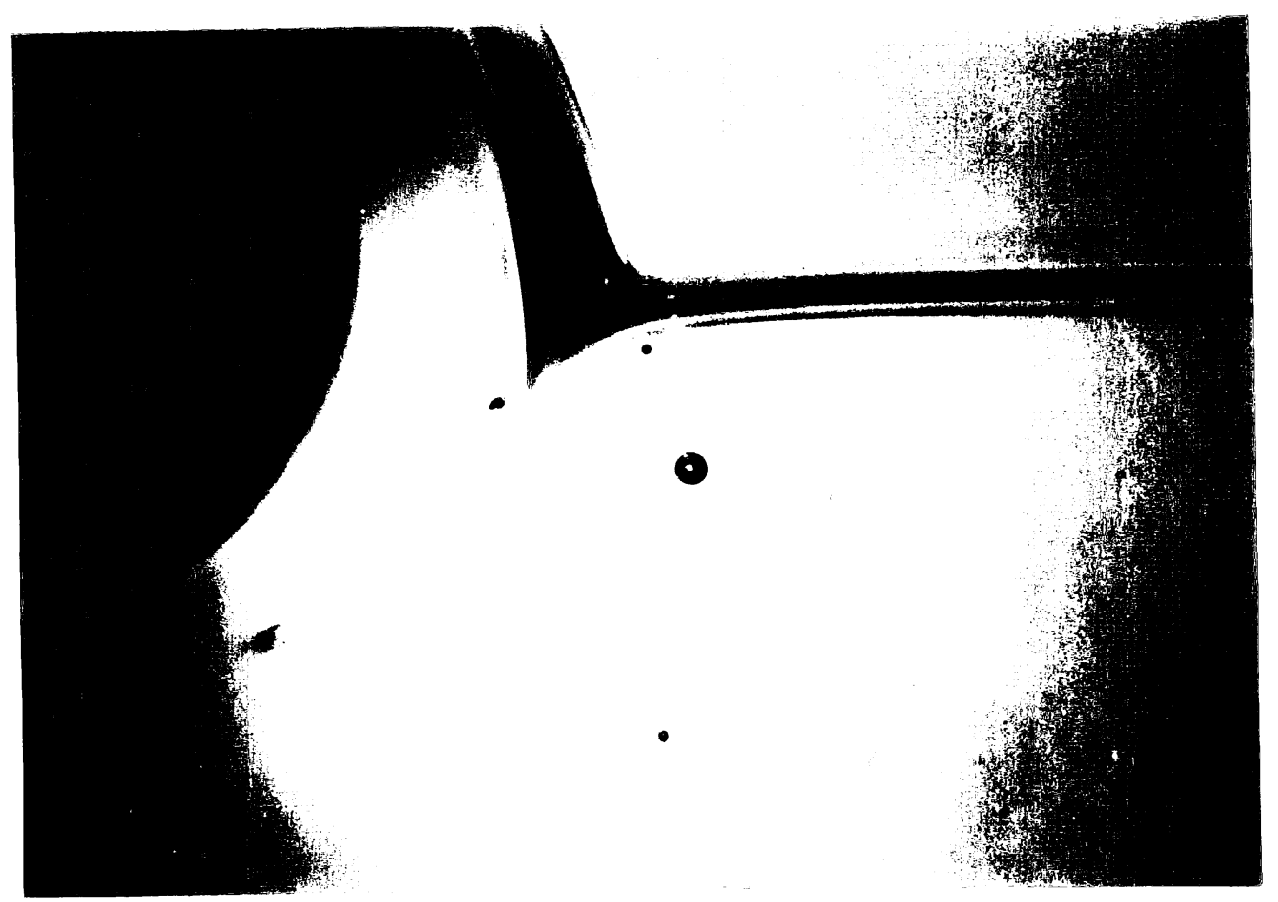

(b)

Figure 13. Critical speed for cusping of M1 in air is approximately 2.4 RPM. a) 2 RPM, b) 75 RPM (cf. Figure 7). 


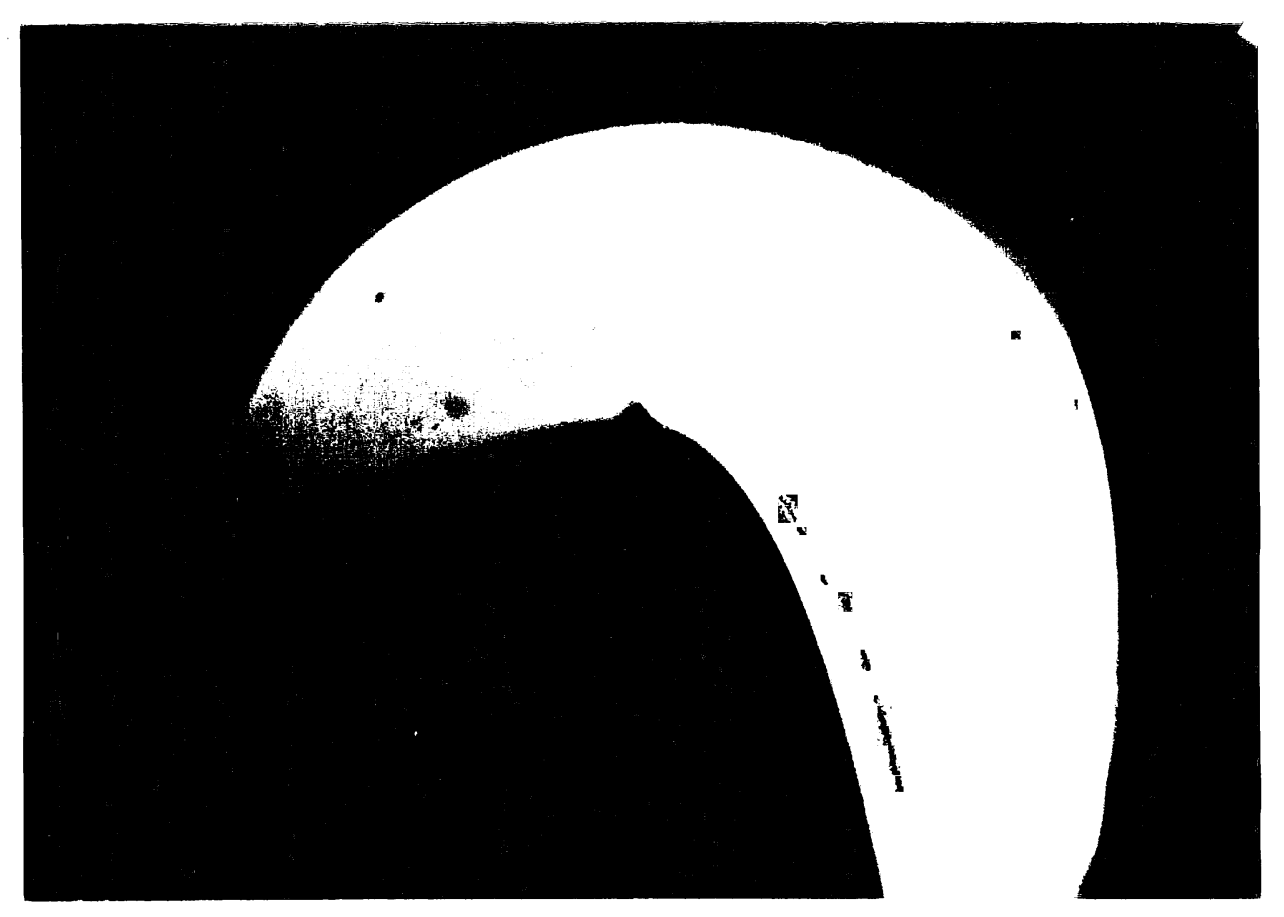

(a)

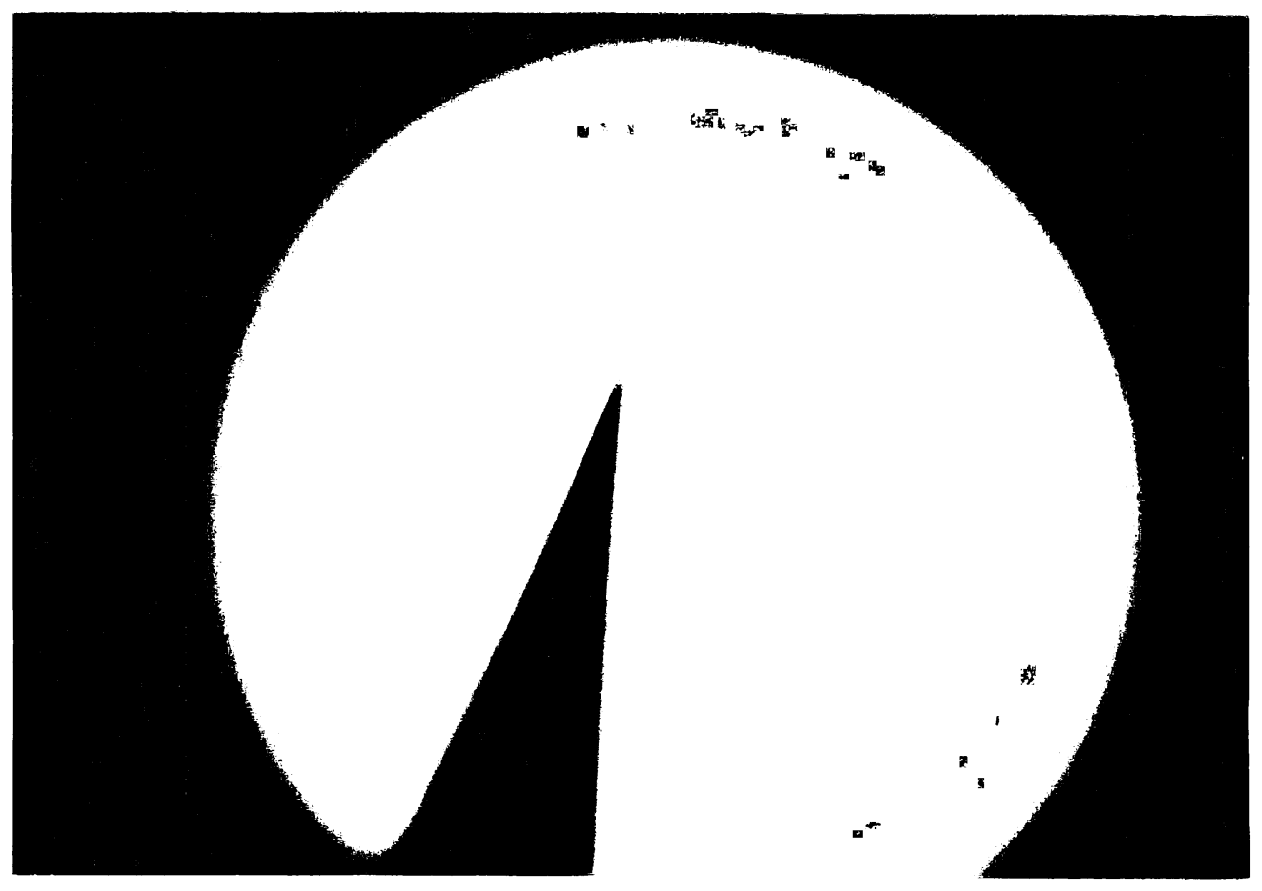

(b)

Figure 14. Critical speed for cusping of STP is approximately 2.9 RPM. a) 2 RPM, the bump on the rounded end is an air bubble emerging through the interface, b) 24 RPM. 


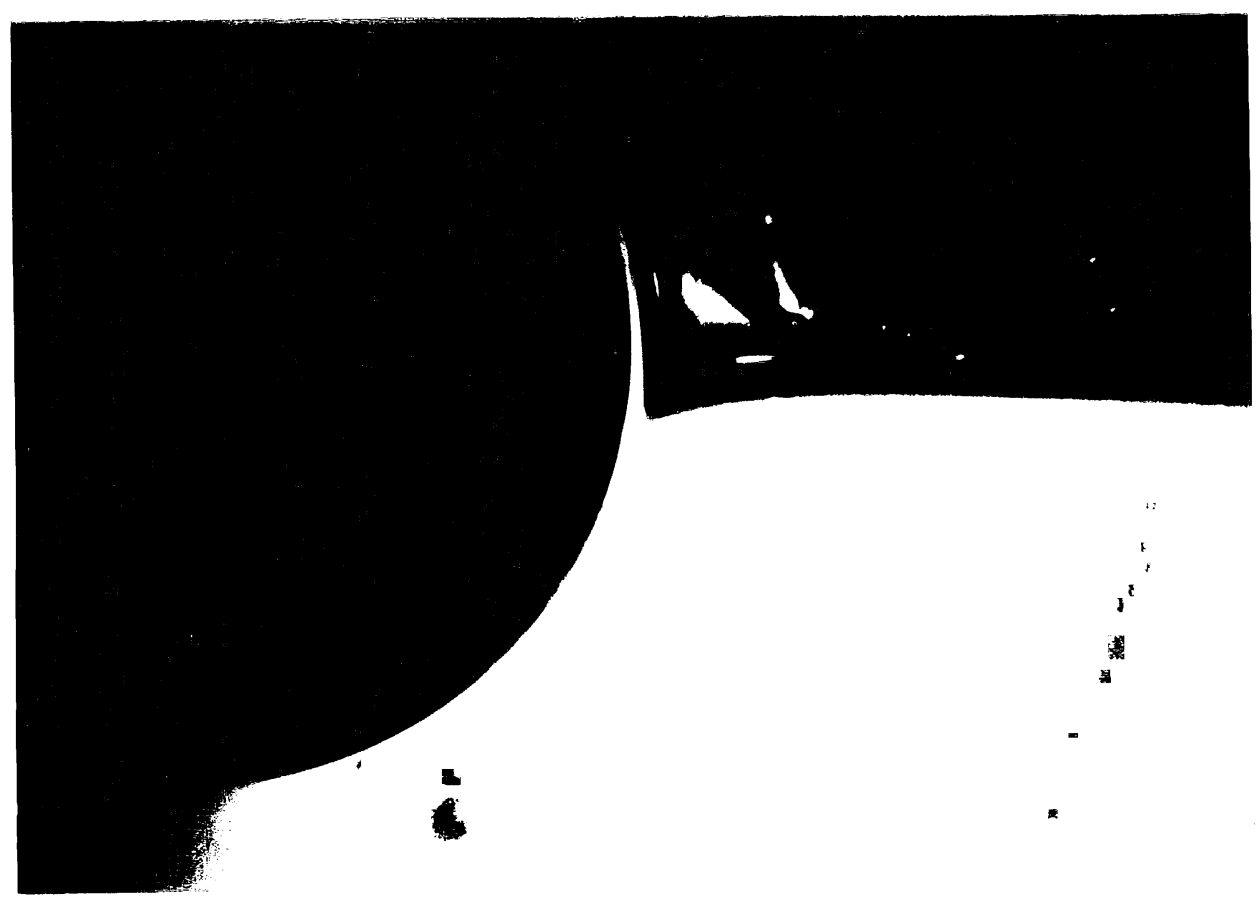

(a)

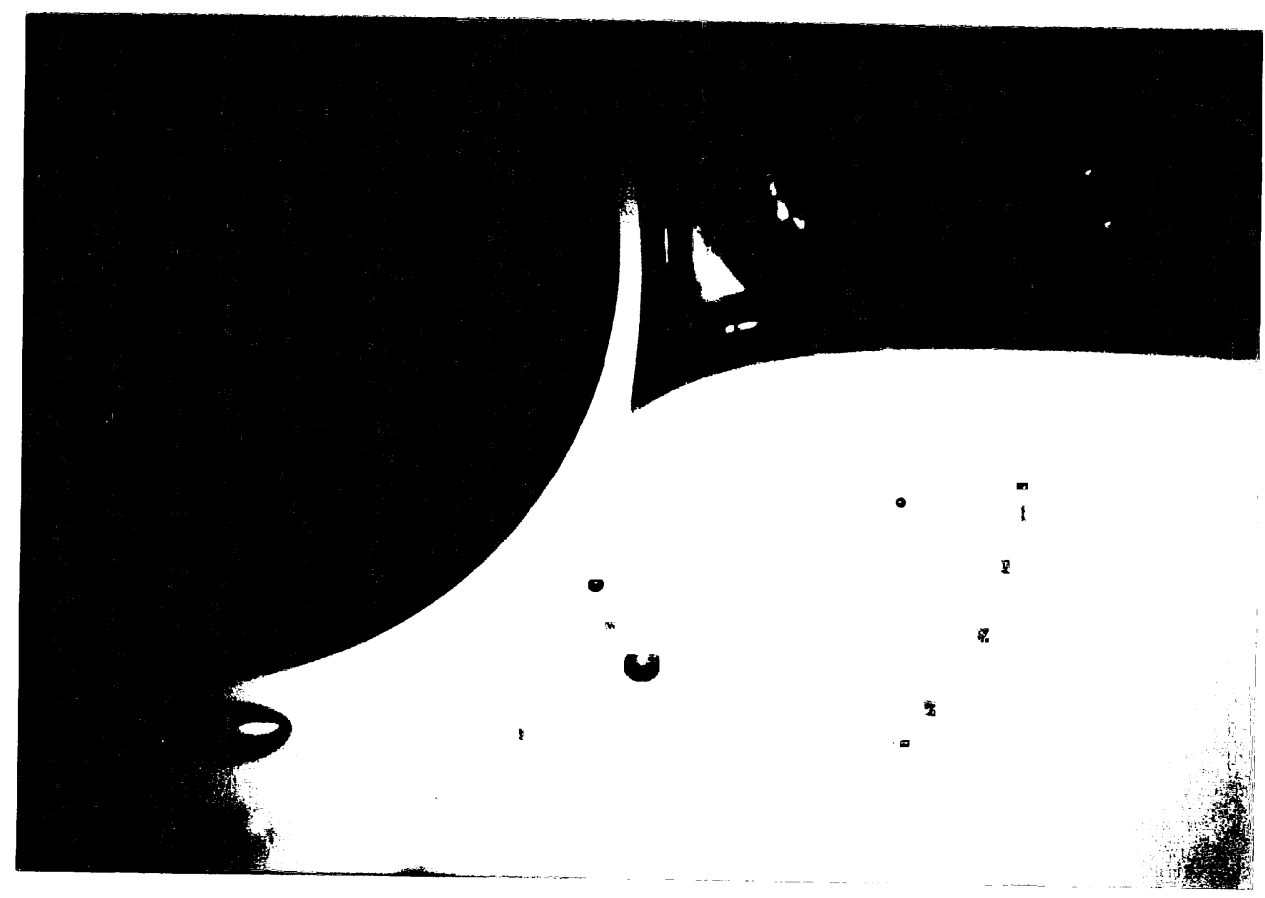

(b) 


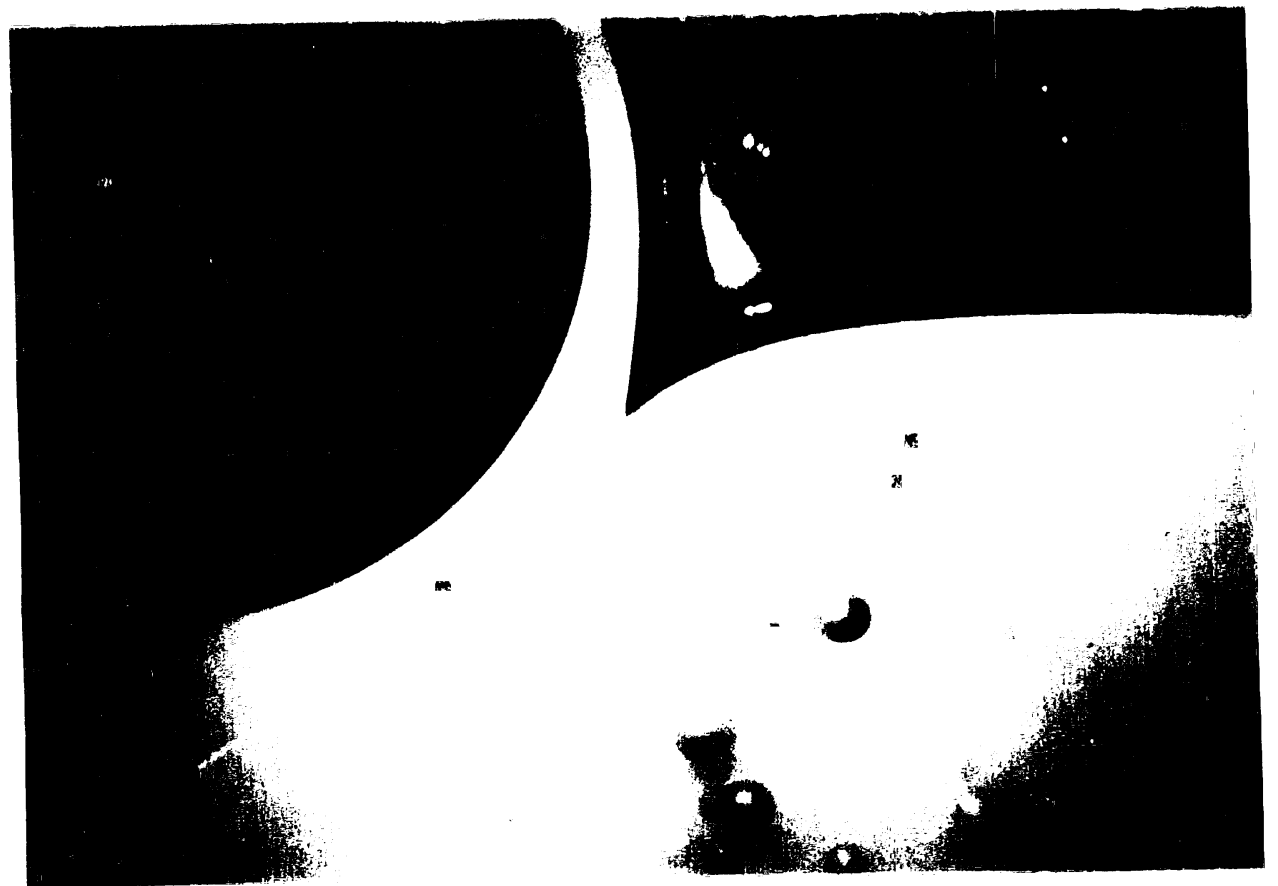

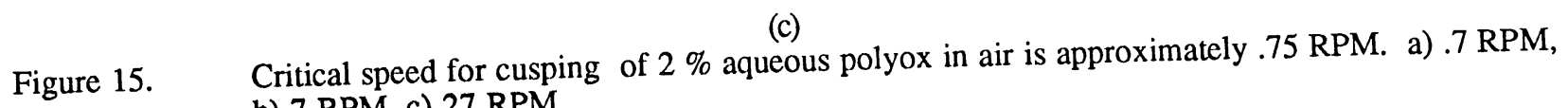
b) 7 RPM, c) 27 RPM. 


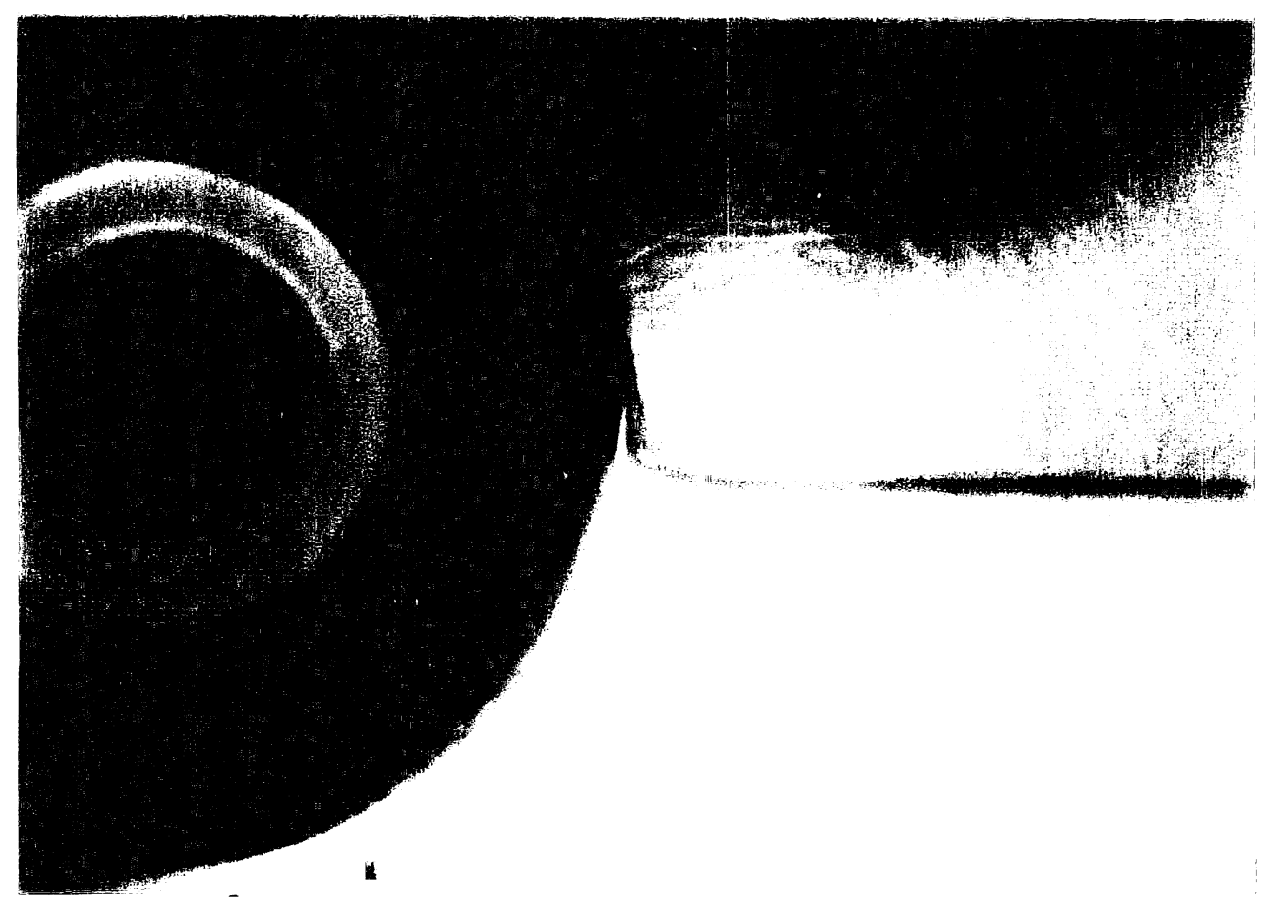

(a)

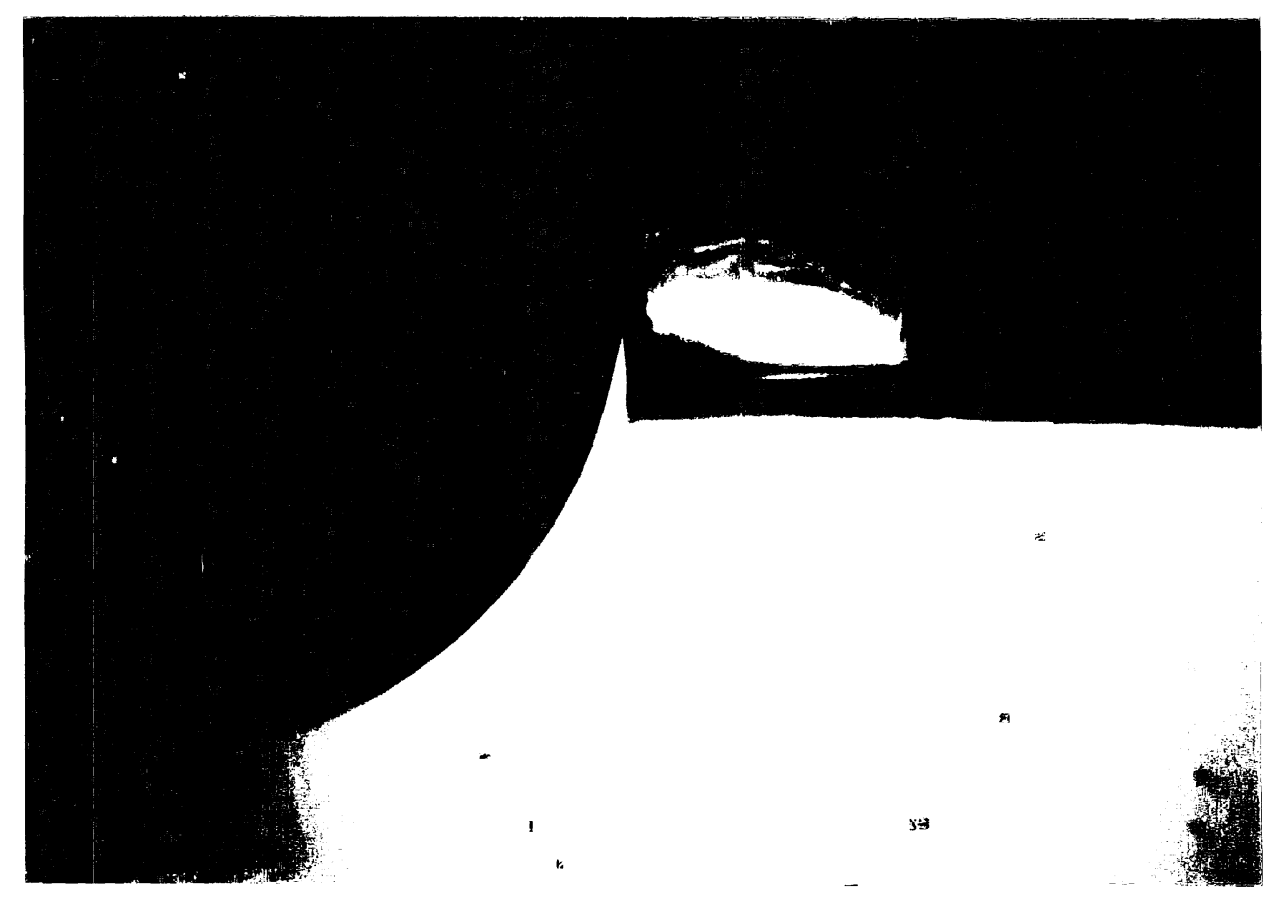

(b) 


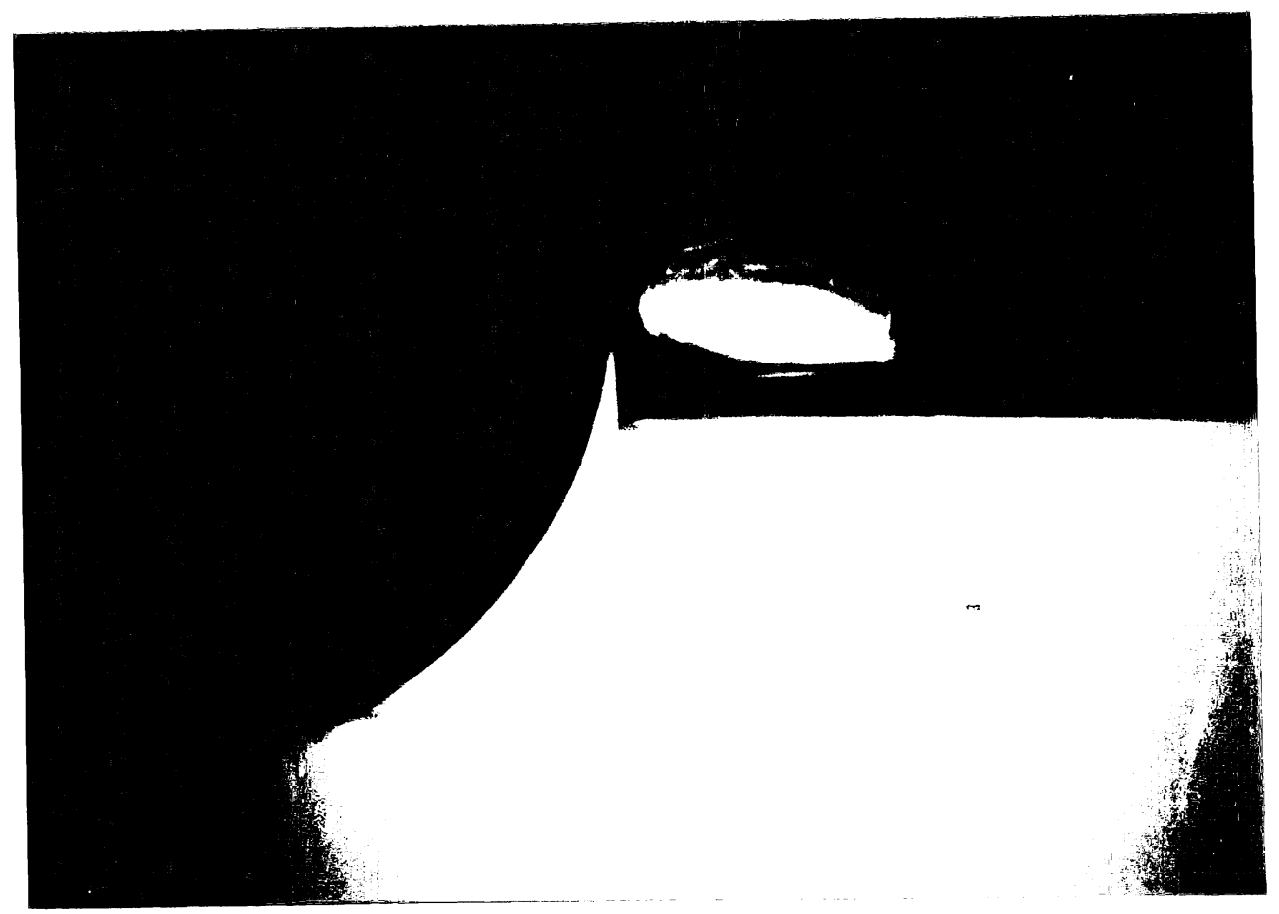

(c)

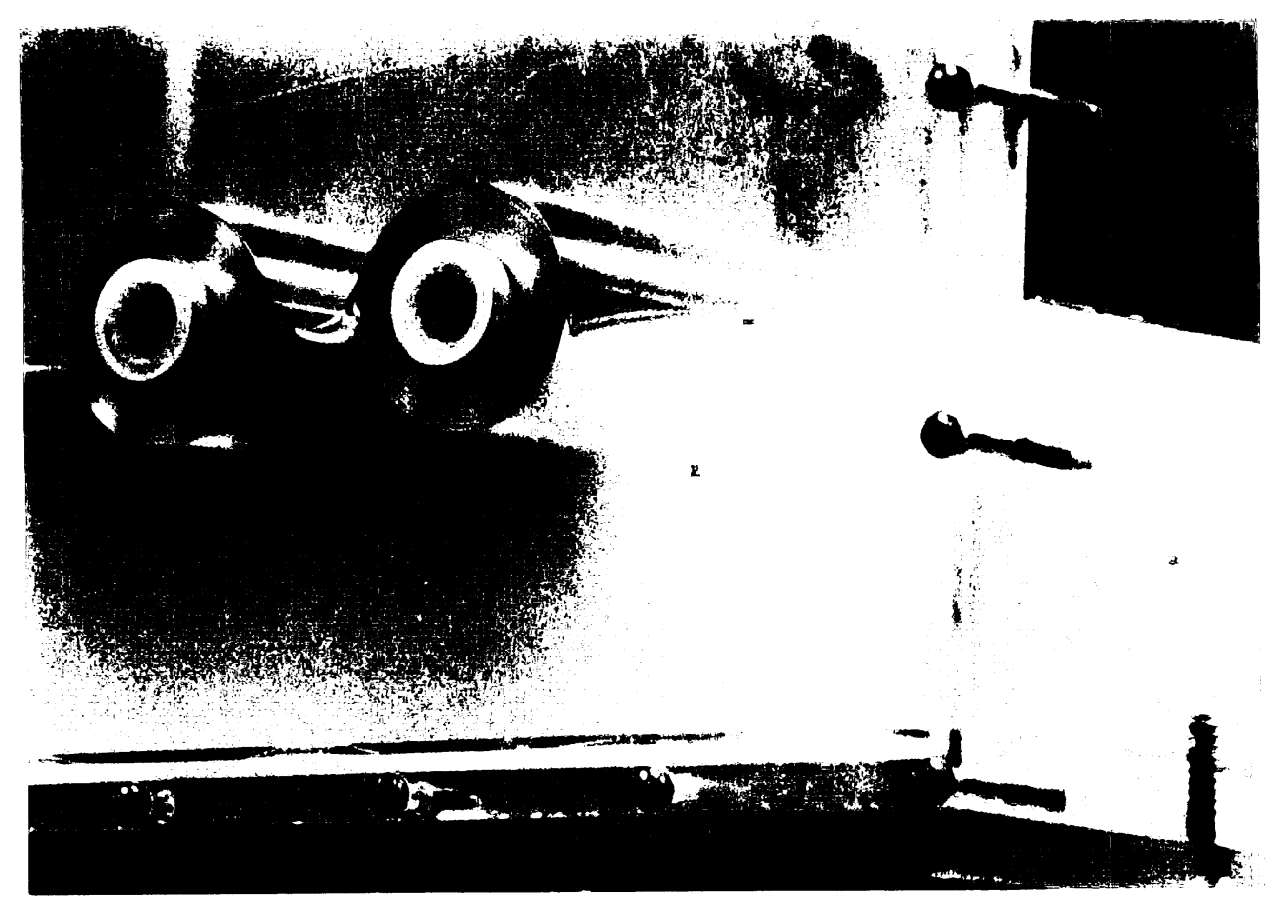

(d)

Figure 16. Critical speed for cusping of $1 \%$ aqueous polyox in air is 51.7 RPM. a) 27 RPM, b) 67 RPM, c) 75 RPM, d) 75 RPM. 


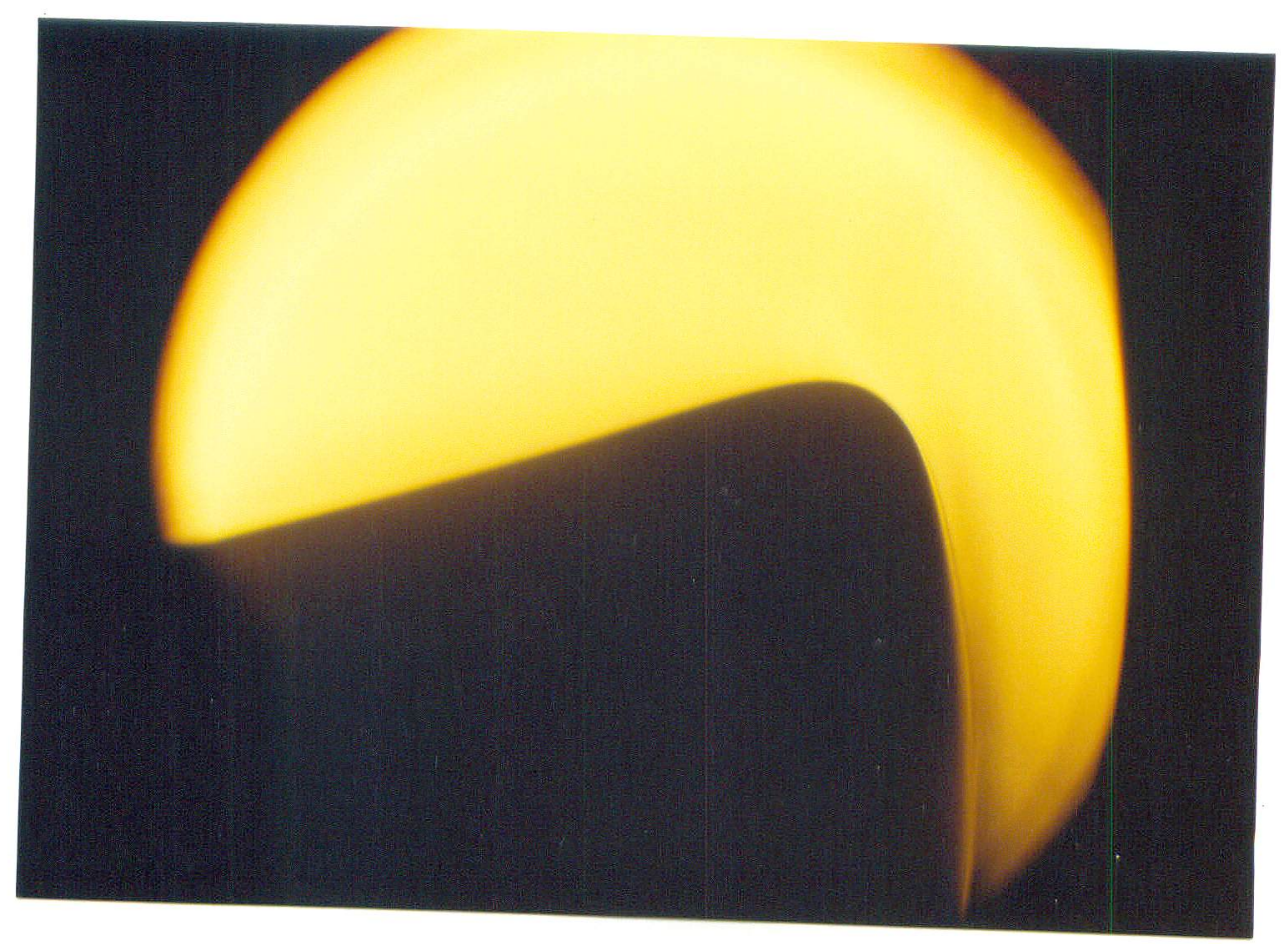

(a)

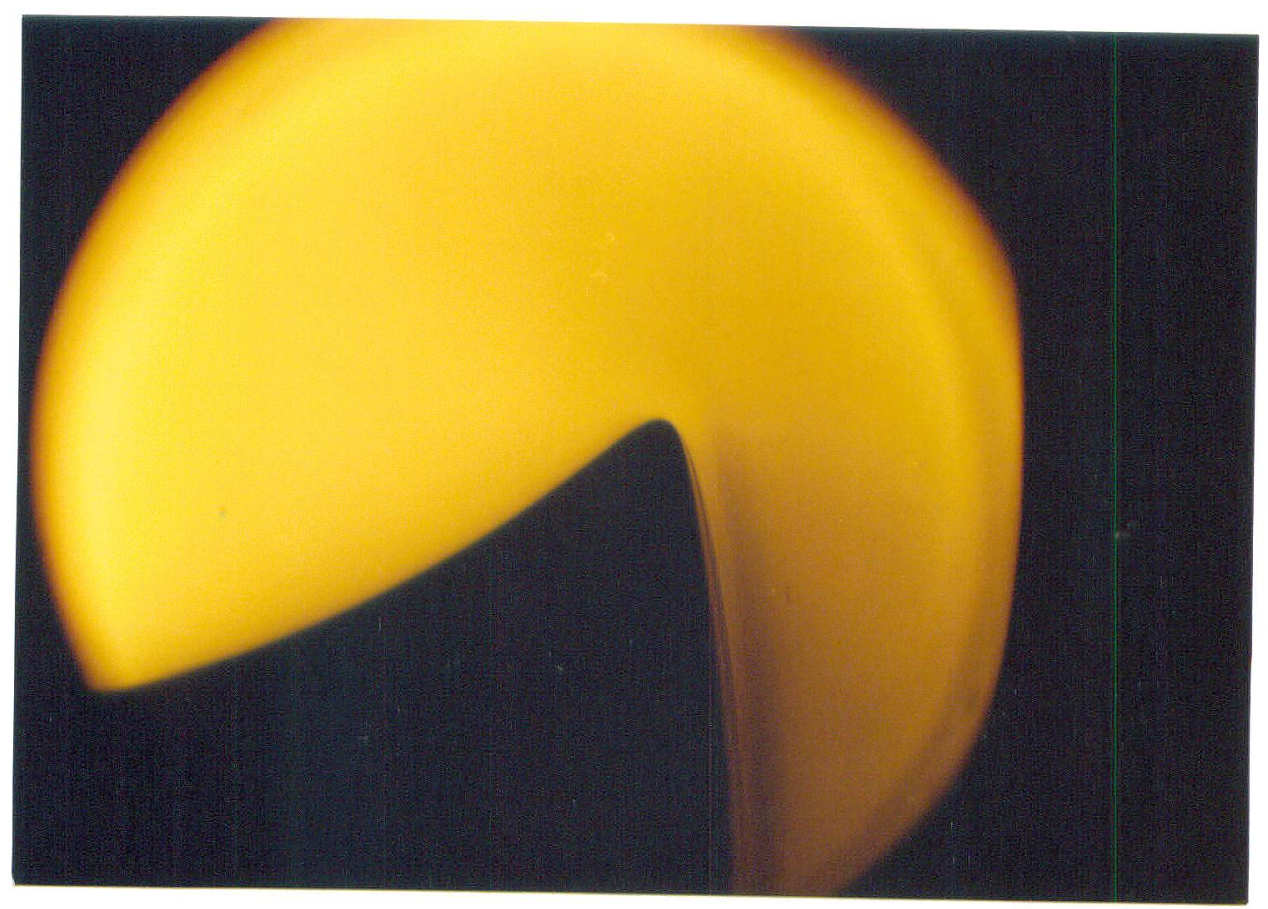

(b) 


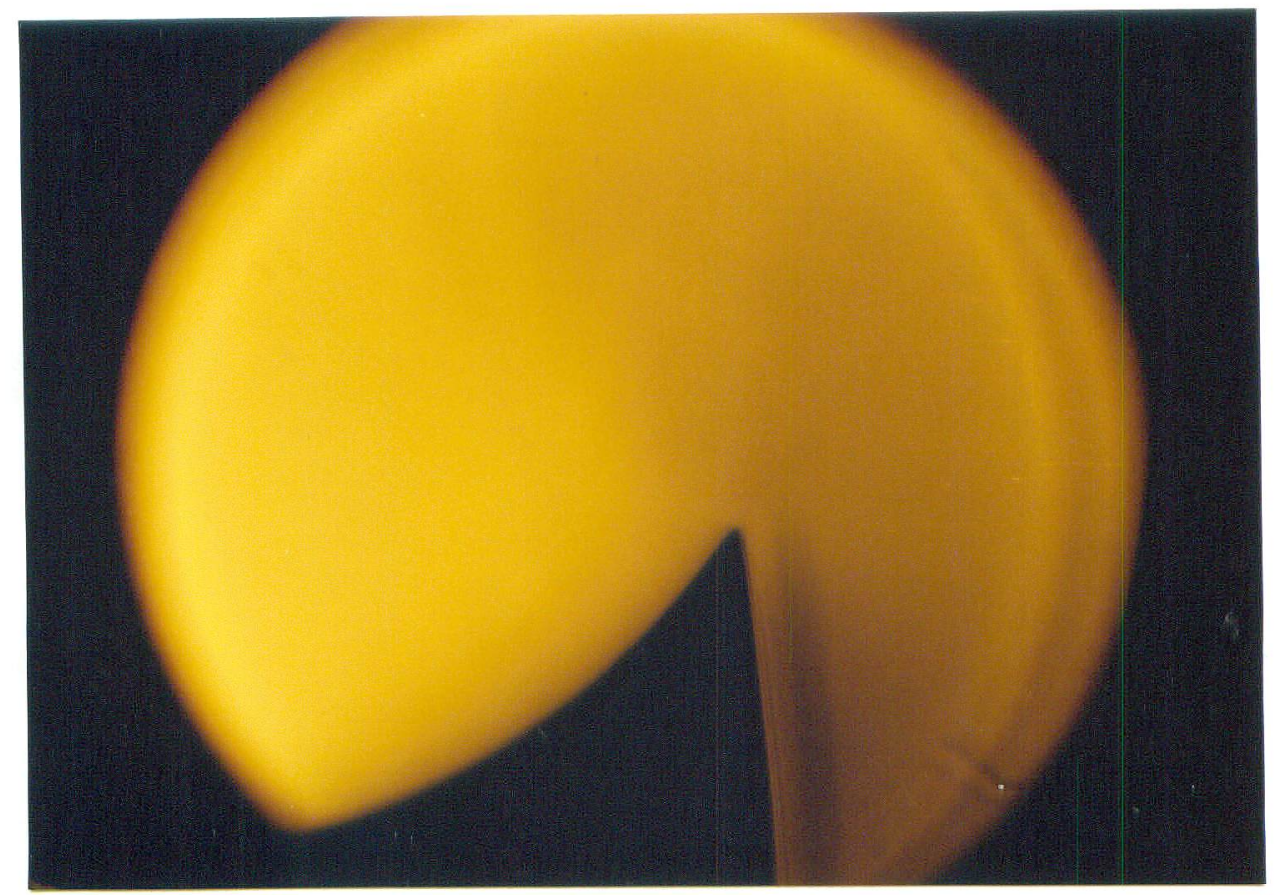

(c)

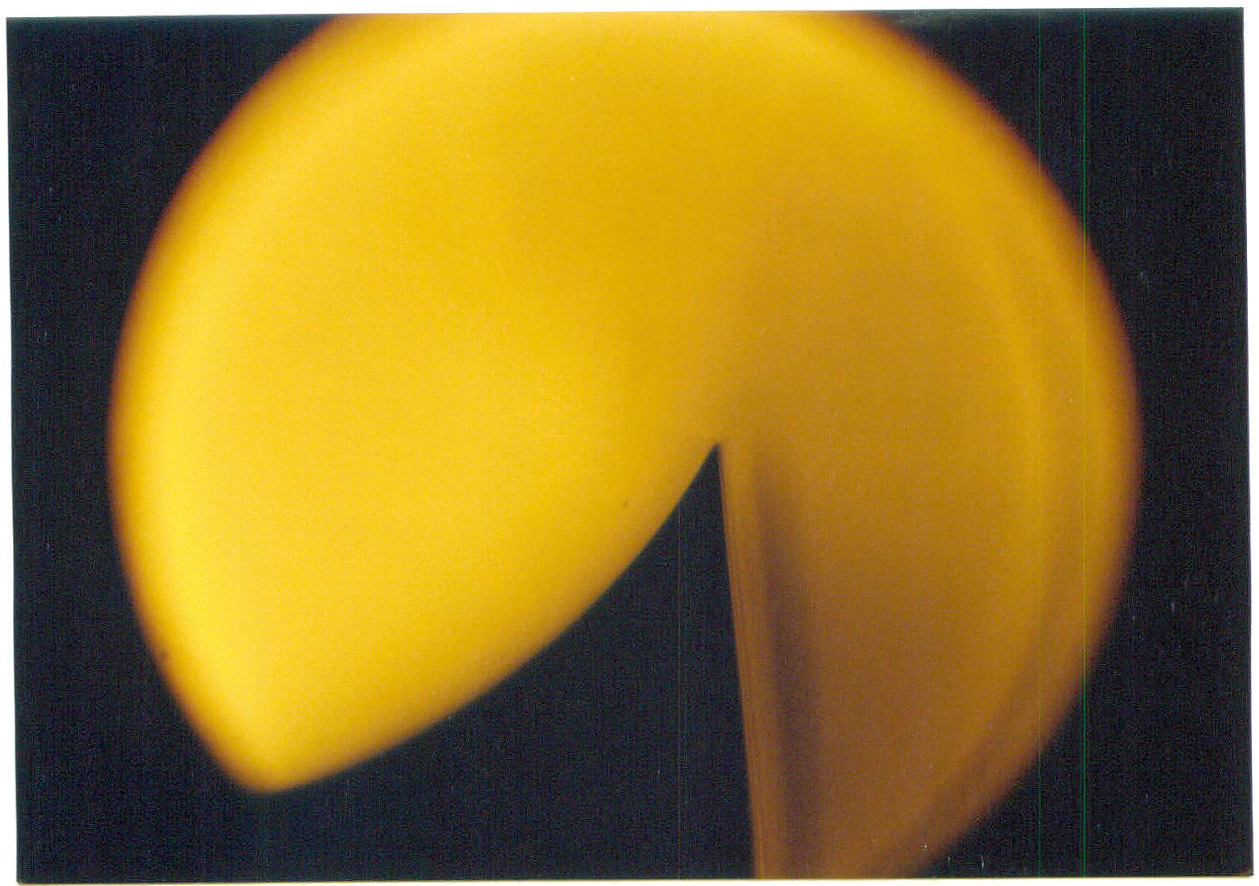

(d) 


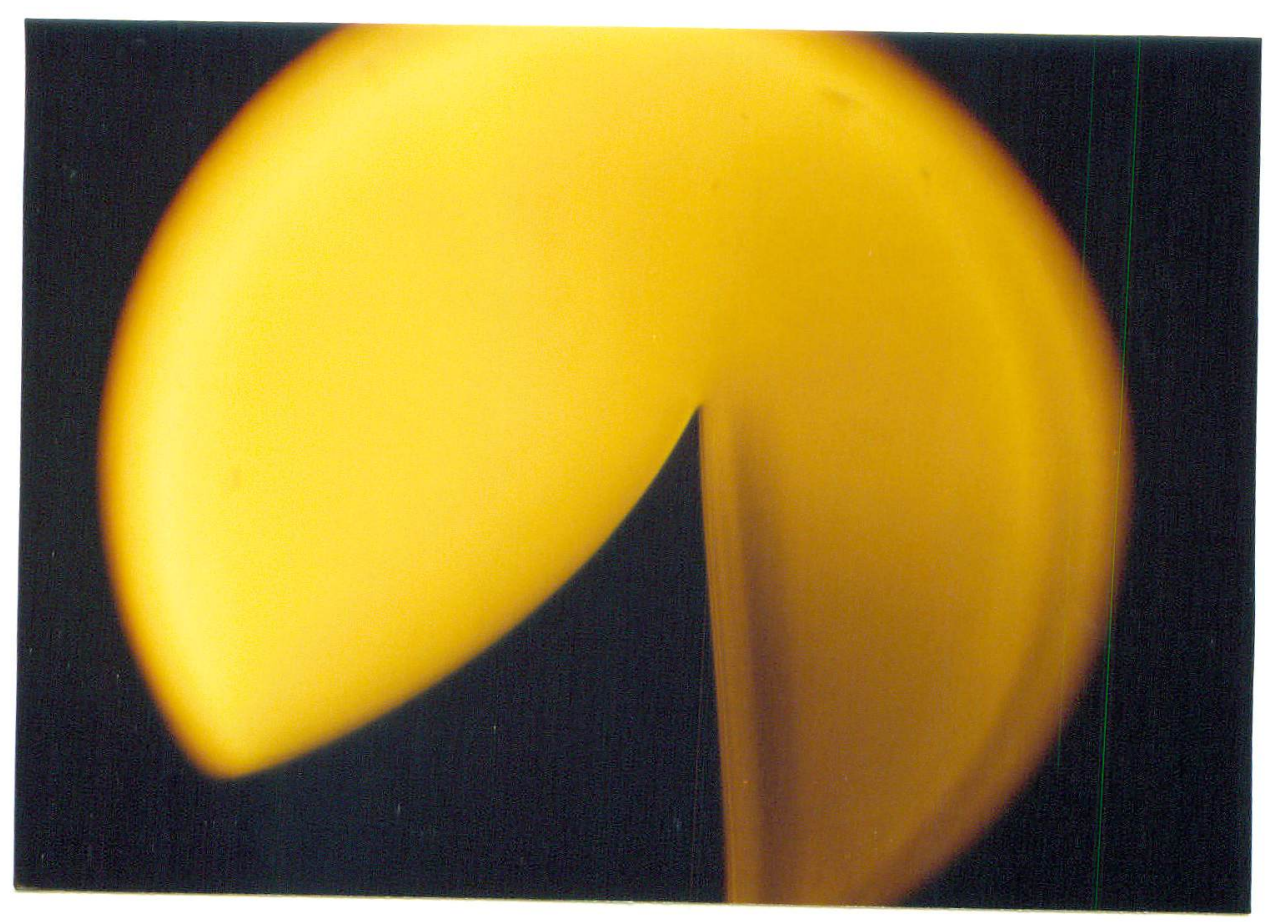

Figure 17. The critical speed for 500 cs. silicone oil is approximately 75 RPM. a) 44 RPM, b) 59 RPM, c)
65 RPM, d) 70 RPM, e) 75 RPM. 

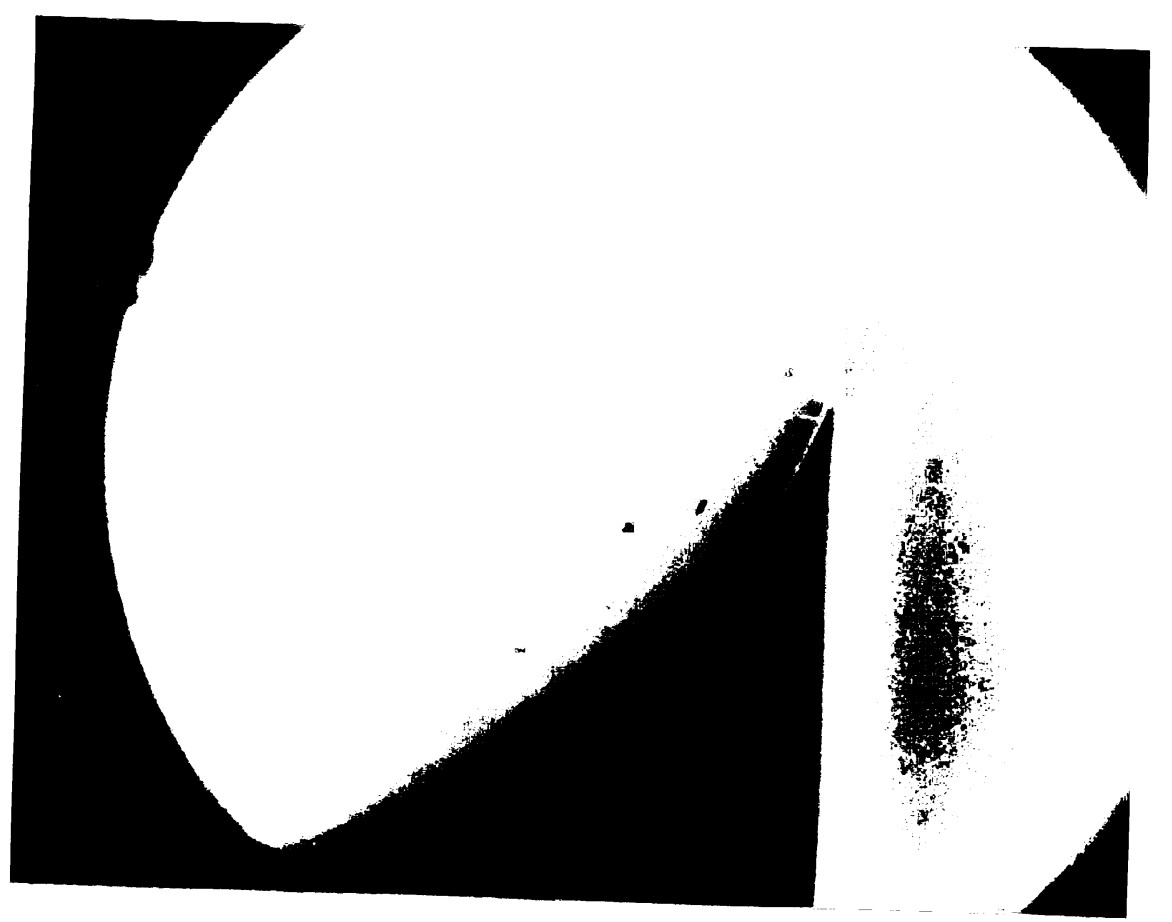

(a)
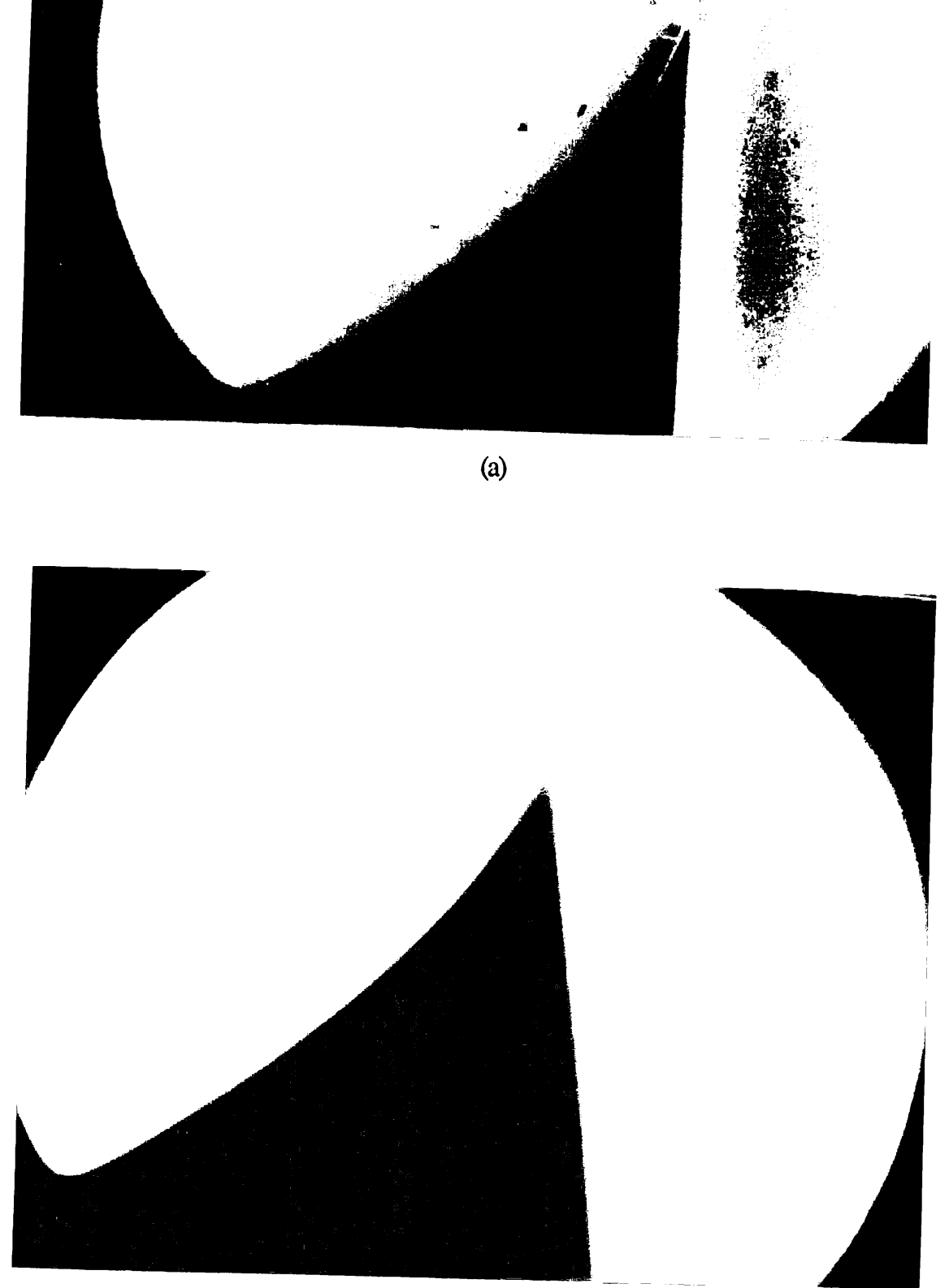

(b) 

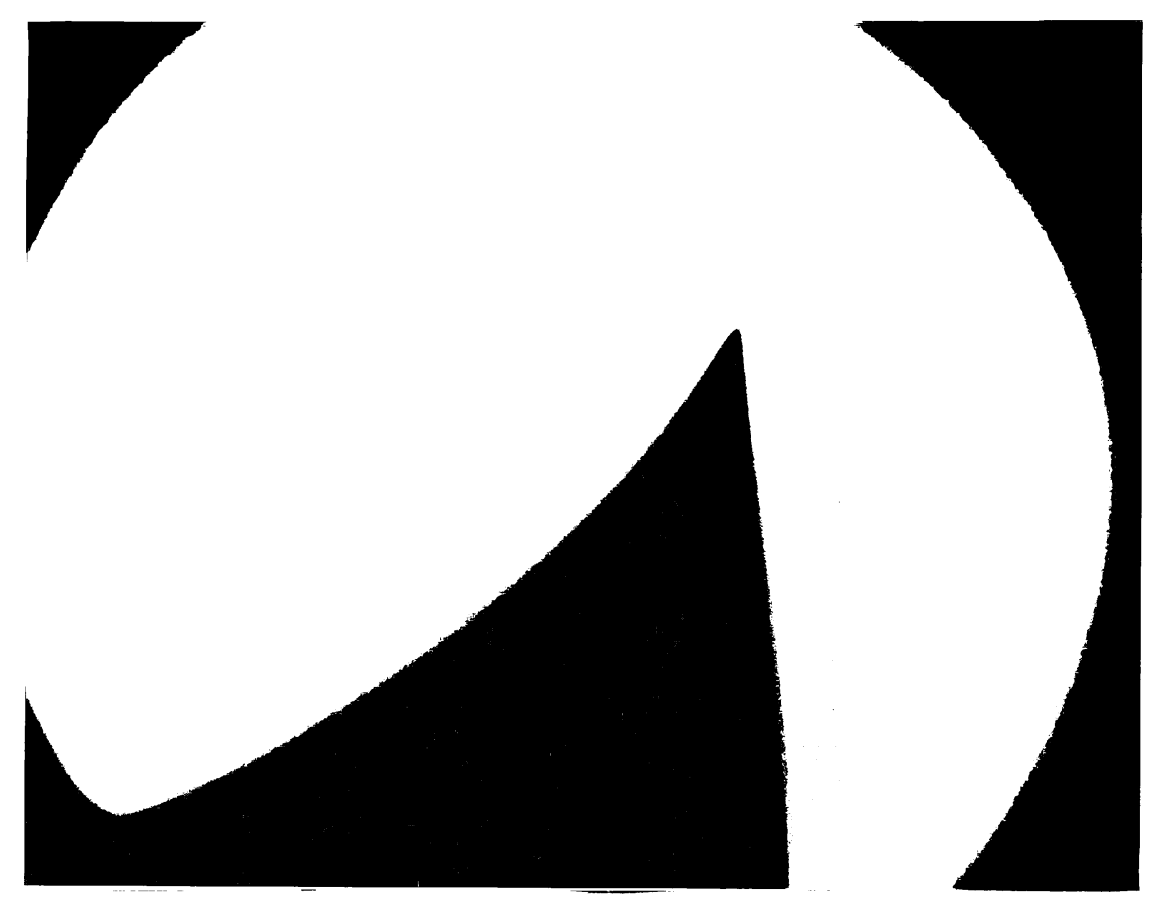

(c)
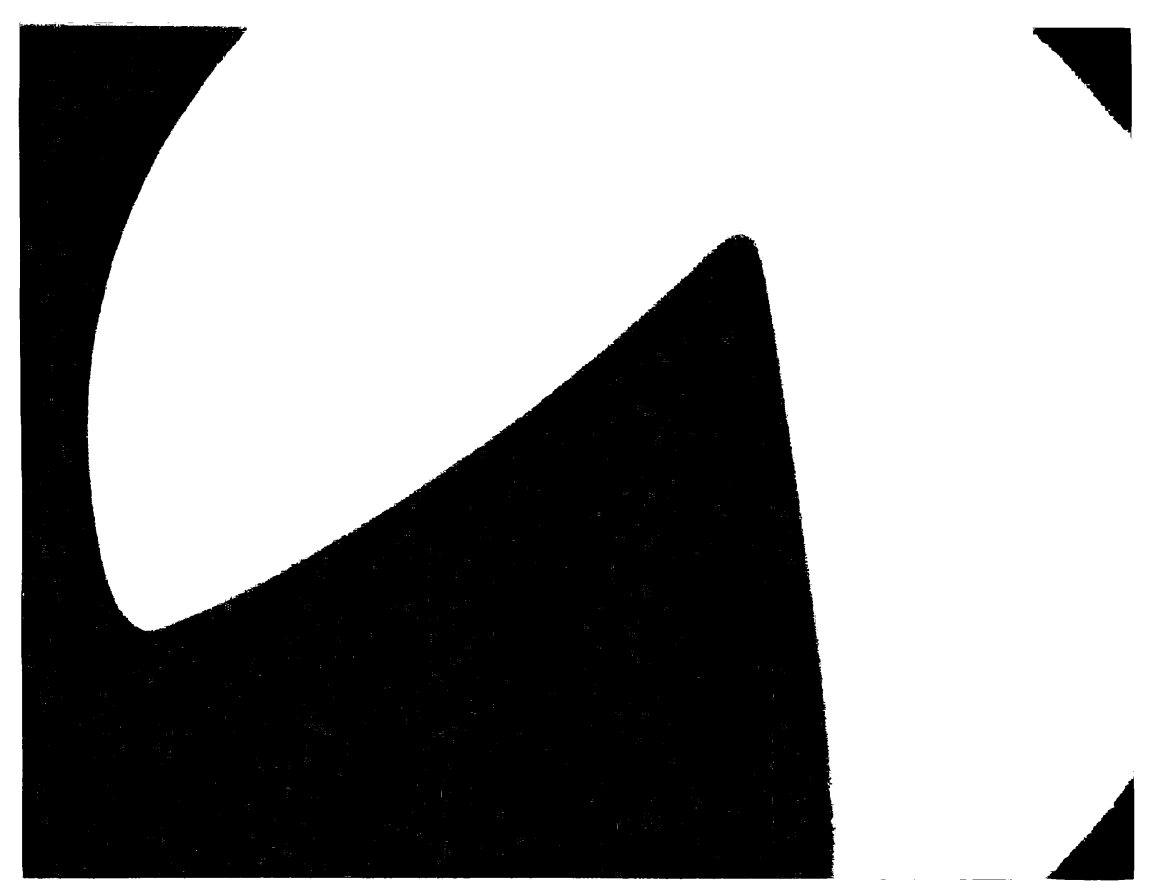

(d)

Figure 18. The critical rotation speed for castor oil is approximately 113 RPM. a) 122 RPM, b) 113 RPM, c) 85 RPM, d) 67 RPM. 

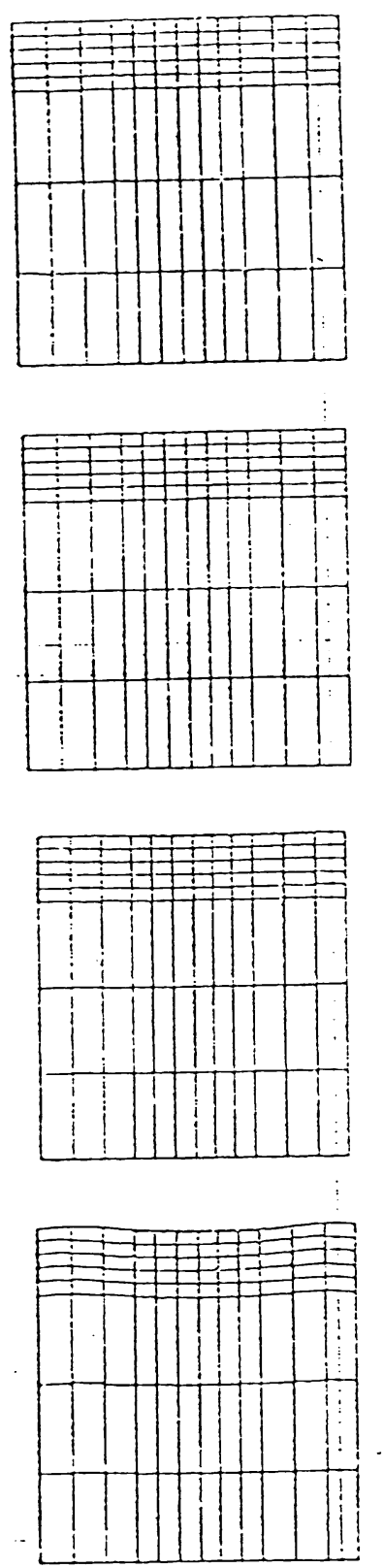

Figure 21. Surface tension zero, times $0.02,0.03,0.26,0.89$. 

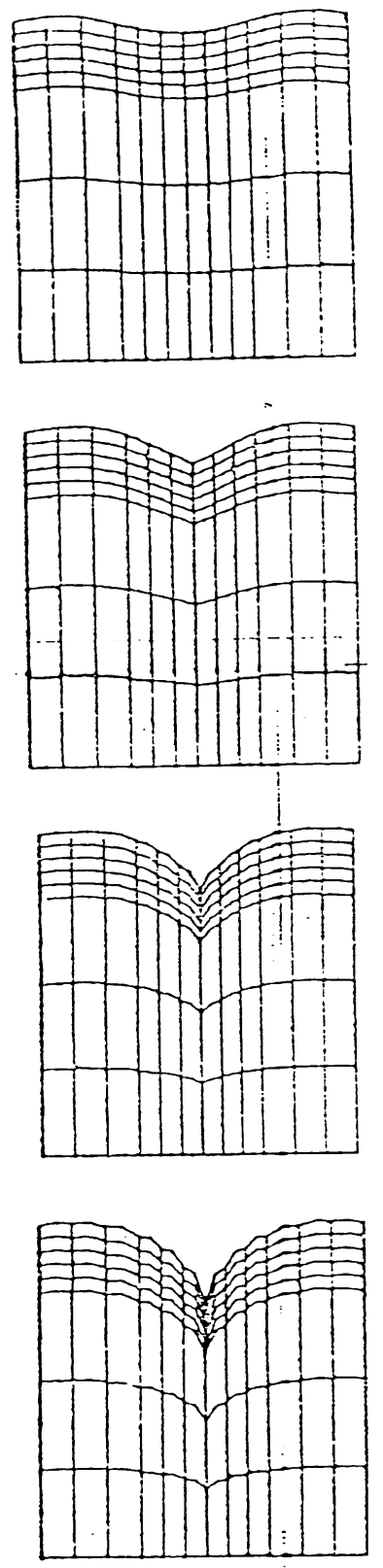

Figure 22. Surface tension zero, times $2.11,3.78,5.60,7.45$. 


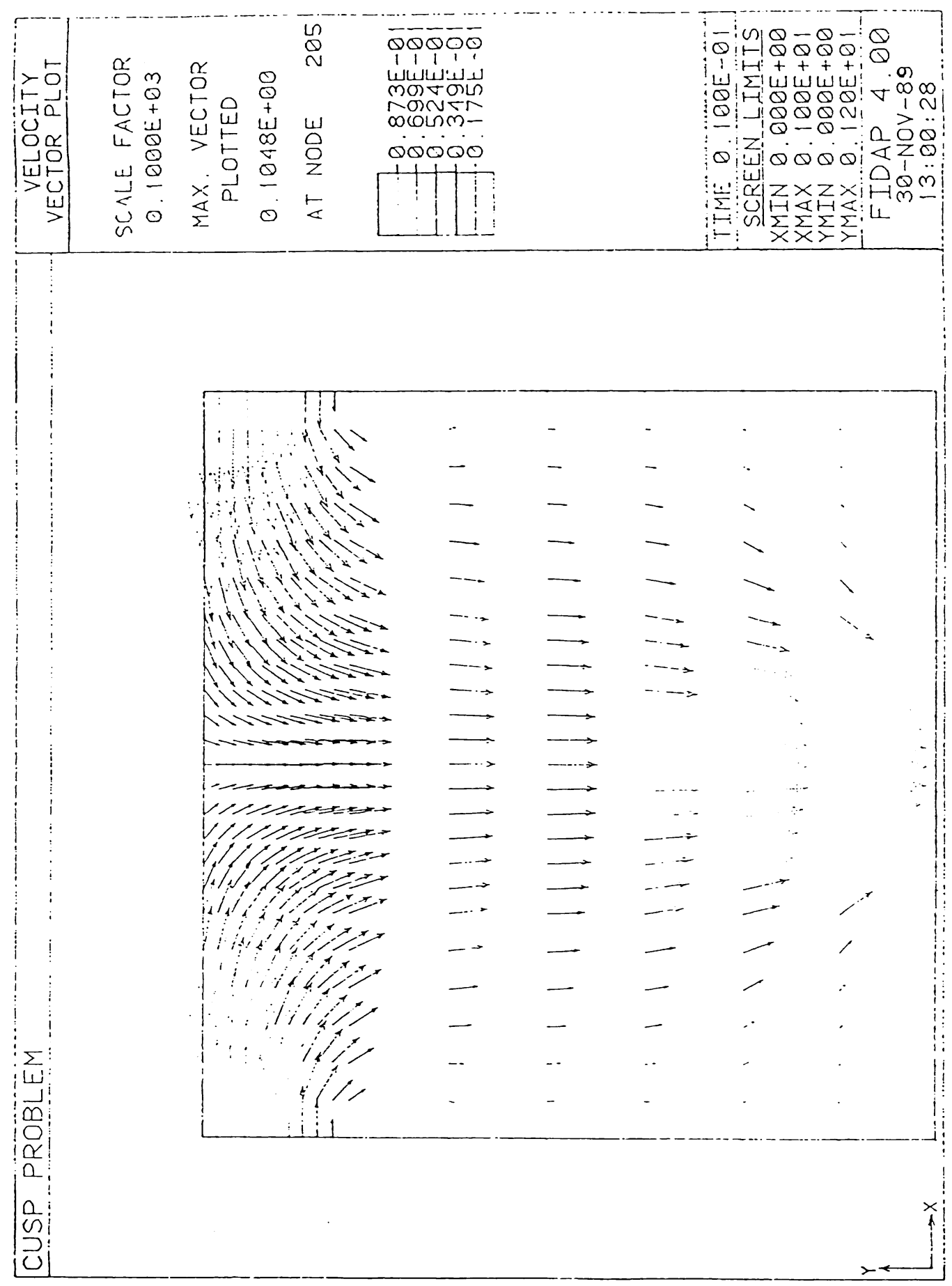

Figure 23. Surface tension zero, time 0.01 . 


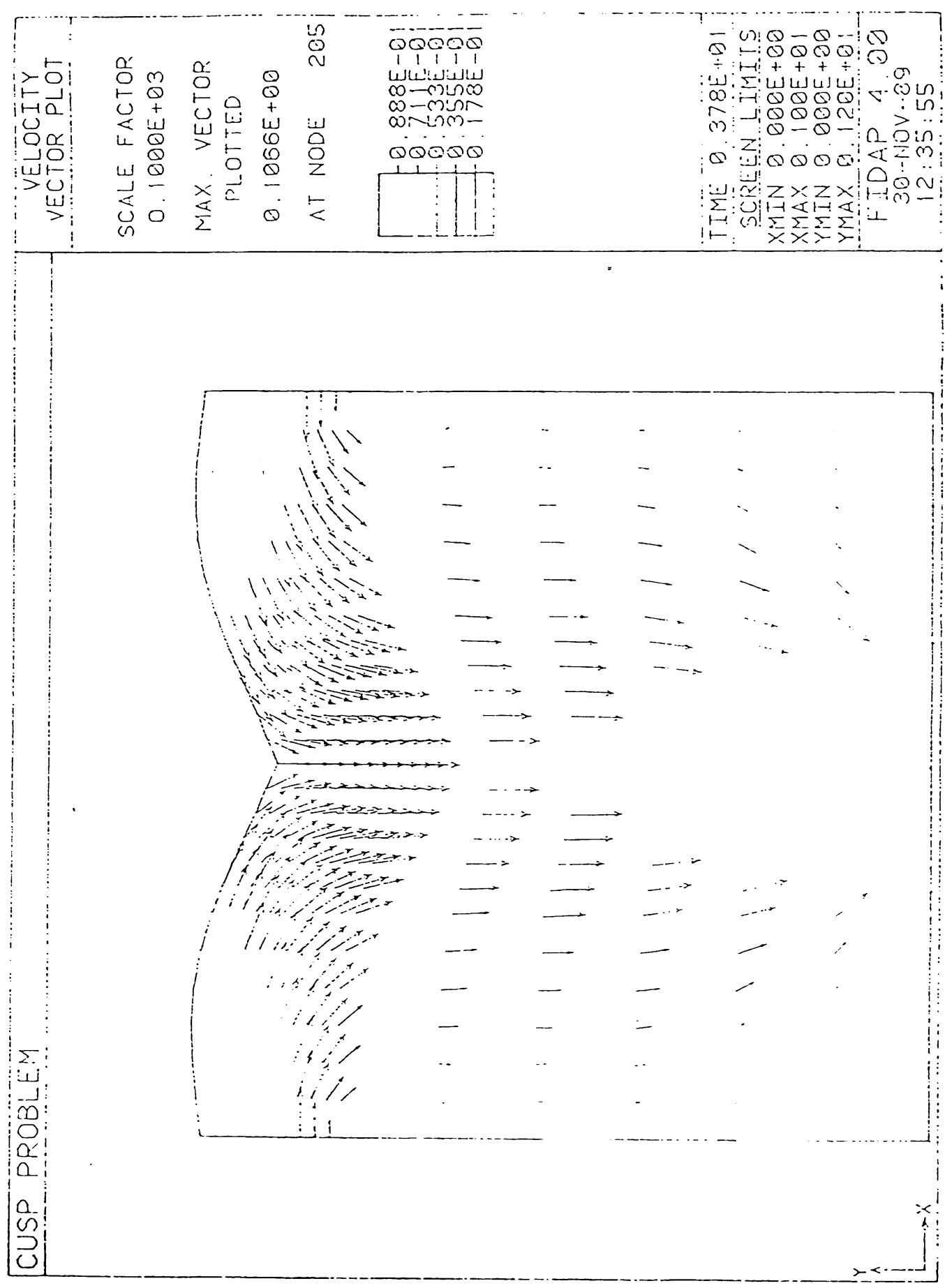

Figure 24. Surface tension zero, time 3.78. 

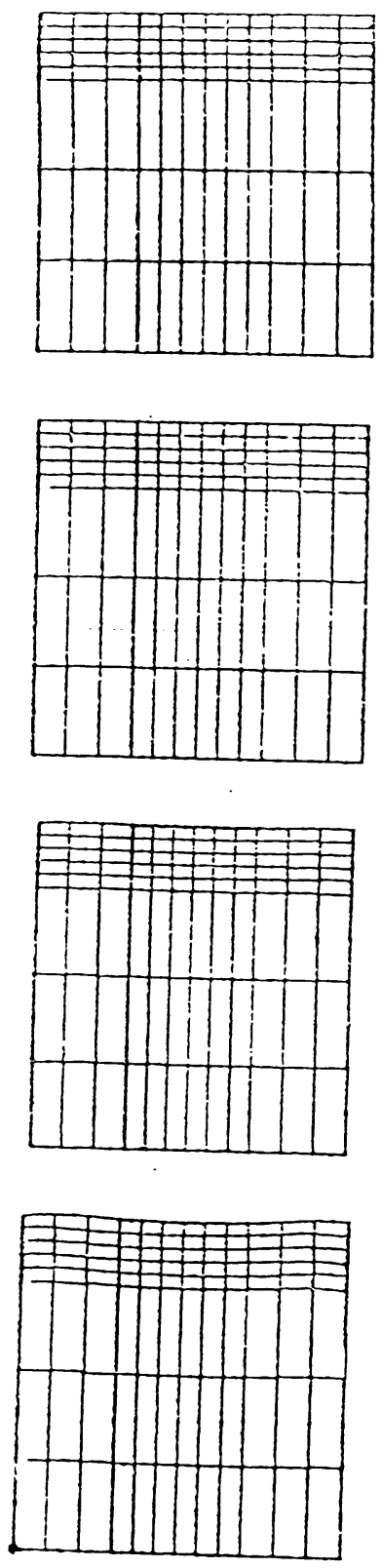

Figure 25(a). Surface tension 0.1, times $0.02,0.03,0.26,0.82$. 
Two-Dimensional Cusped Interfaces
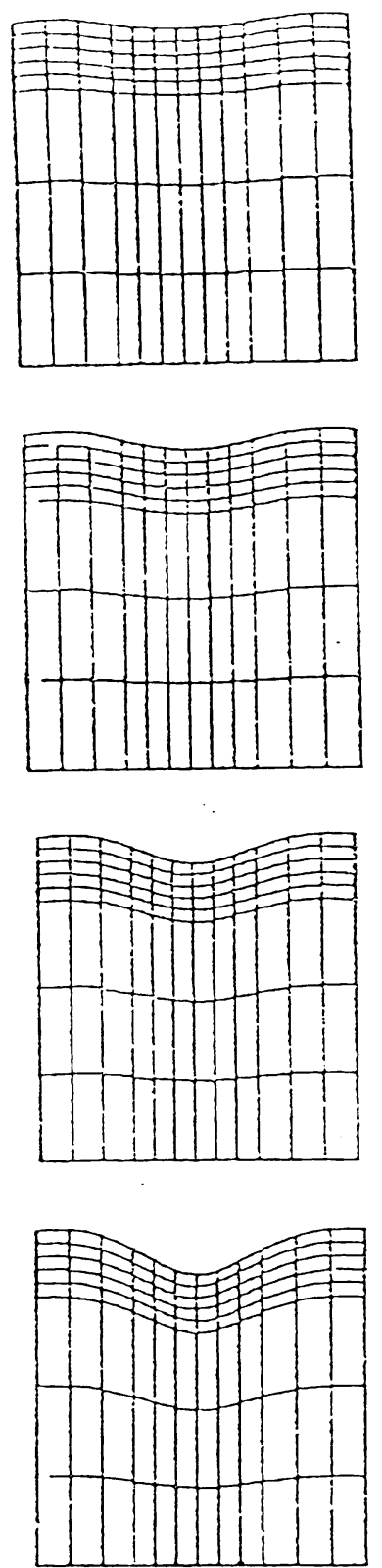

Figure 25(b). Surface tension 0.1, tumes $1.89,3.66,6.66,11.57$. 

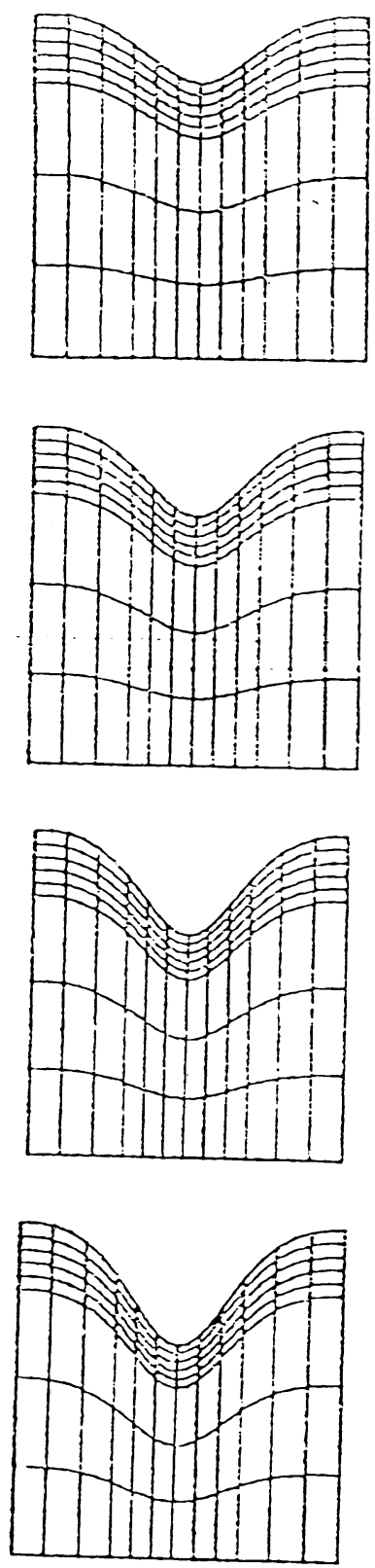

Figure 25(c). Surface tension 0.1, times 19.10, 28.91, 40.31, 53.53. 
Two-Dimensional Cusped Interfaces

Joseph, Nelson, M. \& Y. Renardy
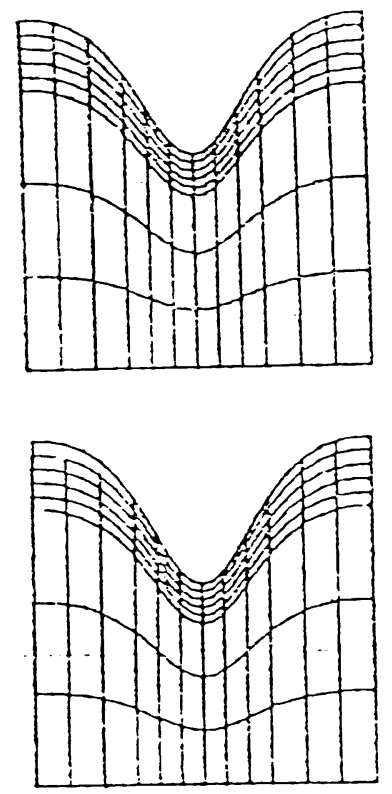

Figure 25(d). Surface tension 0.1, times 69.30, 88.80. 


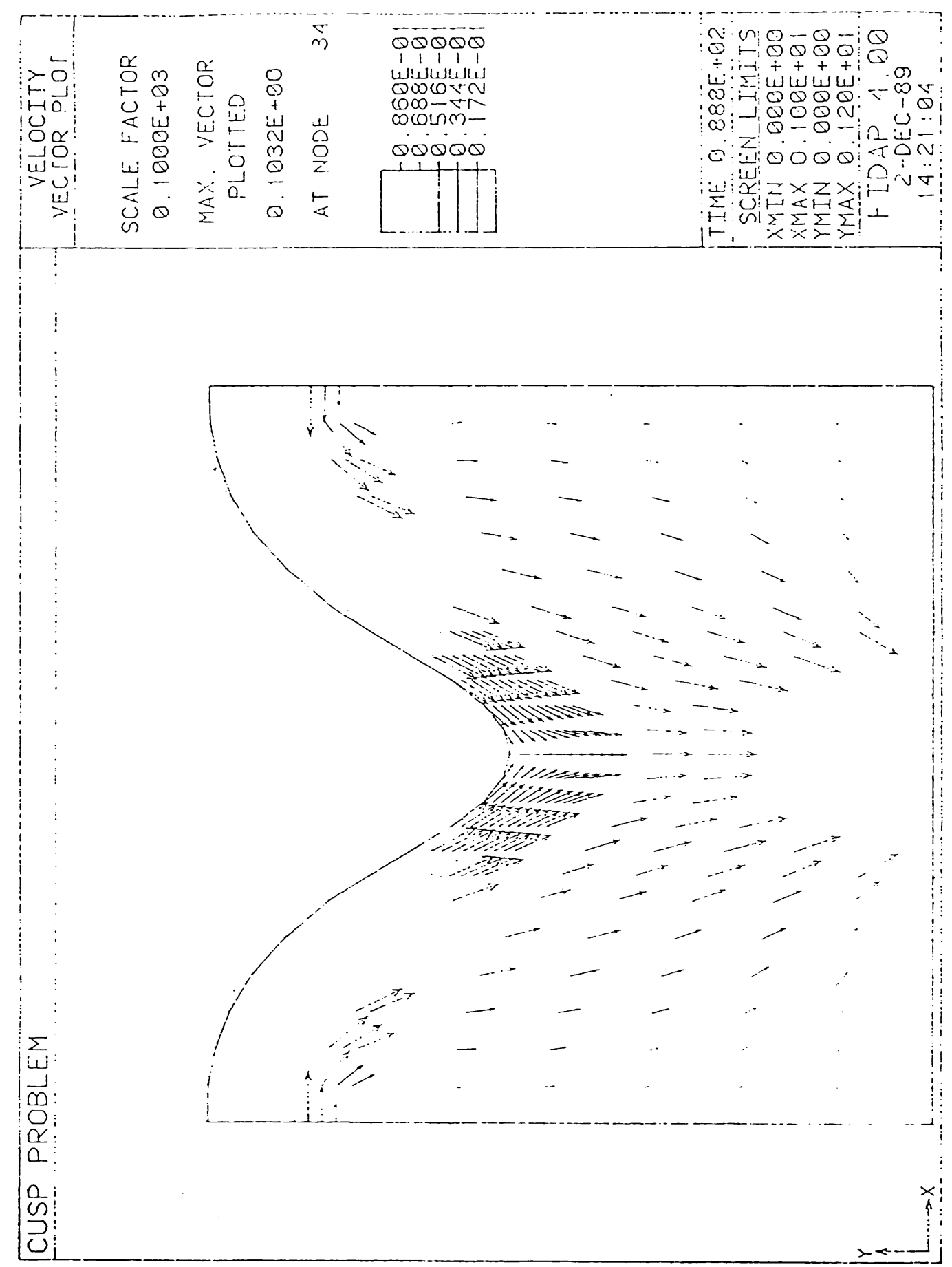

Figure 26. Surface tension 0.1, time 88.80. 

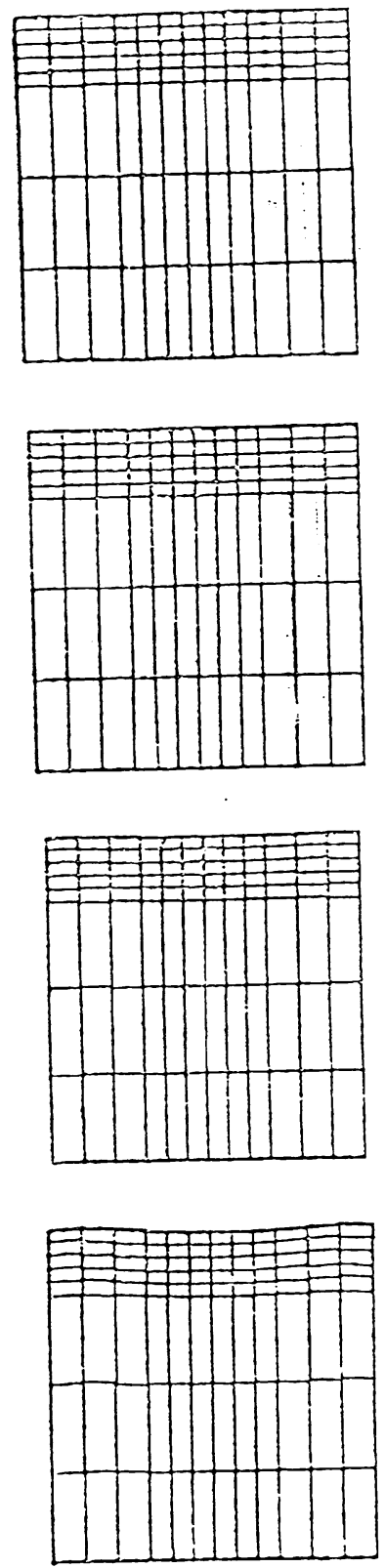

Figure 27(a). Surface tension 0.05 , times $0.02,0.03,0.26,0.86$. 

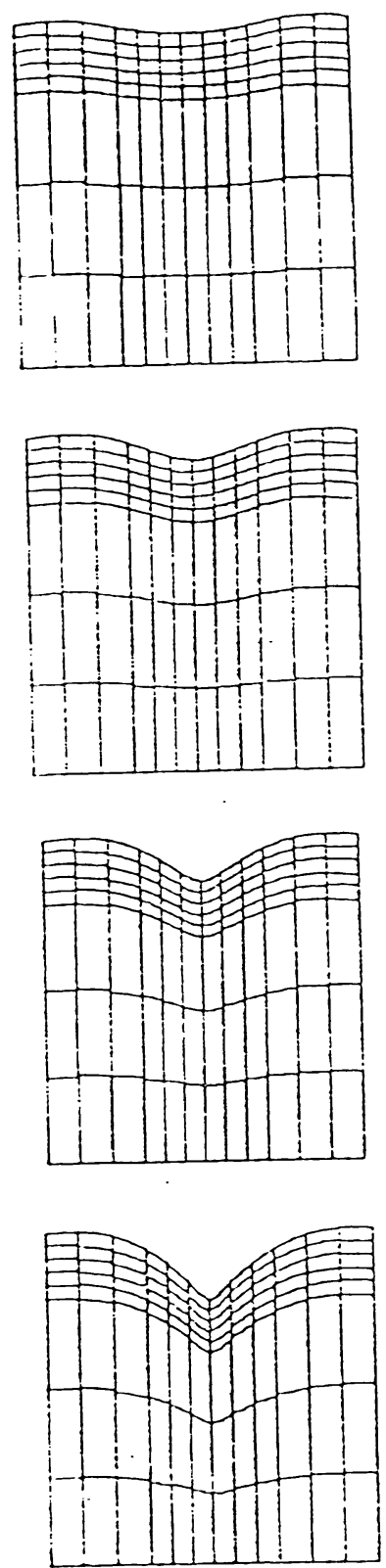

Figure 27(b). Surface tension 0.05, times 2.17, 4.27, 7.52, 12.01 . 

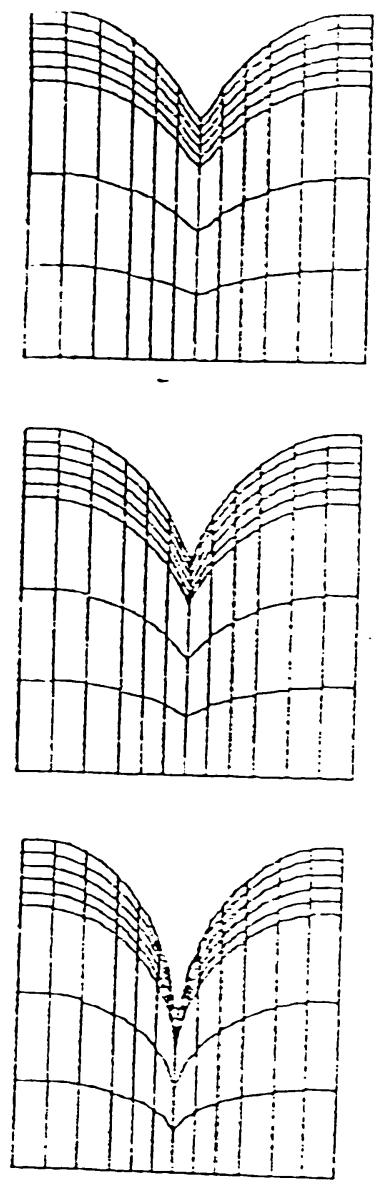

Figure 27(c). Surface tension 0.05, times 17.58, 21.01, 27.2. 


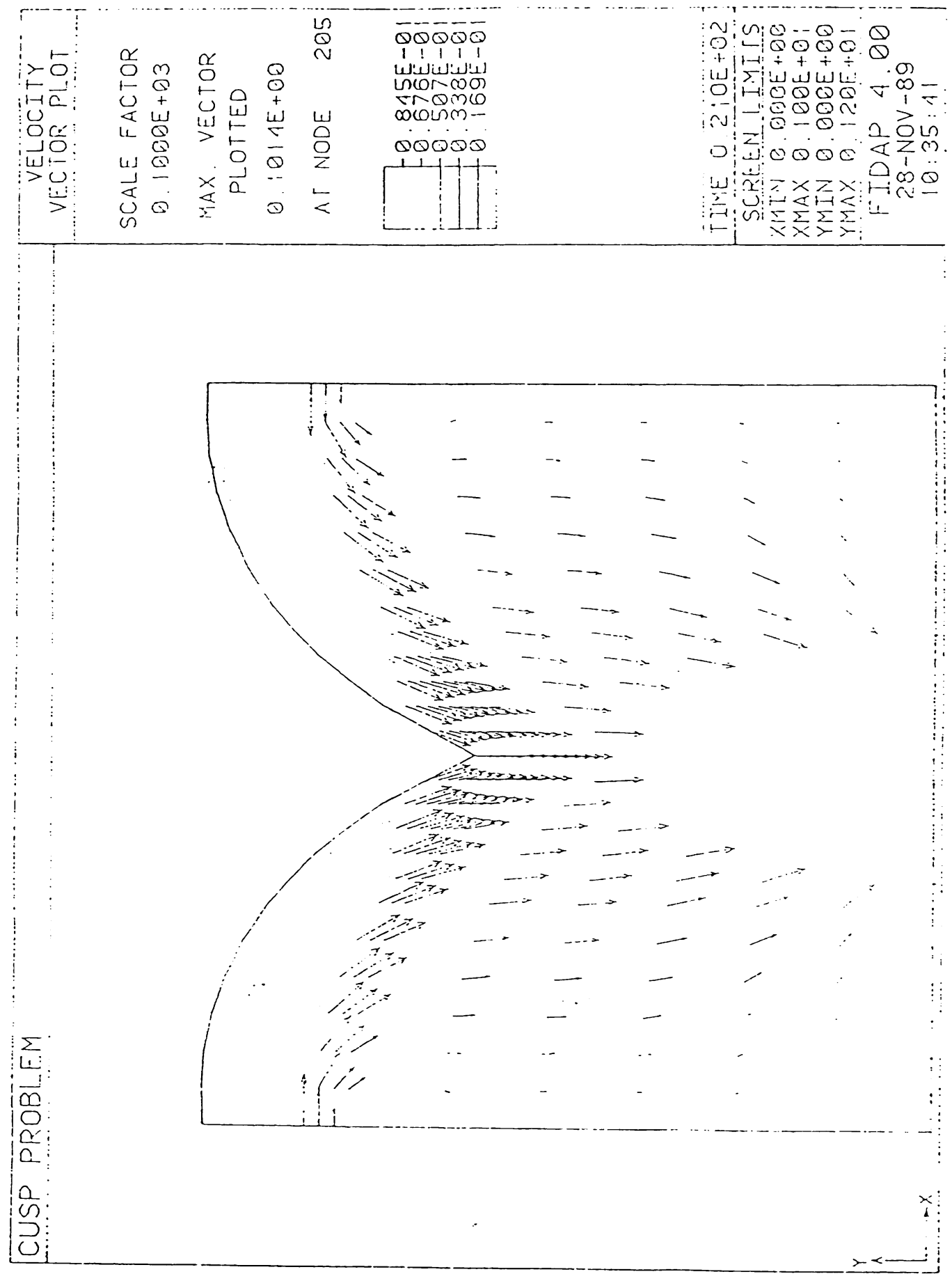

Figure 28. Surface tension 0.05, time 21.01. 


\section{Recent IMA Preprints}

M. Slemrod, Dynamics of Measured Valued Solutions to a Backward-Forward Heat Equation

A vner Friedman and Jürgen Sprekels, Steady States of Austenitic-Martensitic-Domains in the Ginzburg-Landau Theory of Shape Memory Alloys

Avner Friedman and Bei Hu, Degenerate Hamilton-Jacobi-Bellman Equations in a Bounded Domain

E.G. Kalnins, Willard Miller, Jr., and M.V. Tratnik, Families of Orthogonal and Biorthogonal Polynomials on the N-Sphere

Heinrich Freistühler, On Compact Linear Degeneracy

Matthew Witten, Quantifying the Concepts of Rate and Acceleration/Deceleration of Aging

J.P. Albert and J.L. Bona, 'Total Positivity and the Stability of Internal Waves in Stratified Fluids of Finite Depth

Brian Coomes and Victor Zurkowski, Linearization of Polynomial Flows and Spectra of Derivations

Yuriko Renardy, A Couette-Poiseuille Flow of Two Fluids in a Channel

Michael Renardy, Short wave instabilities resulting from memory slip

Daniel D. Joseph and Michael Renardy, Stokes' first problem for linear viscoelastic fluids with finite memory

Xiaxi Ding, Superlinear Conservation Law with Viscosity

J.L. Ericksen, Liquid Crystals with Variable Degree of Orientation

F. Robert Ore, Jr. and Xinfu Chen, Electro-Optic Modulation in an Arbitrary Cross-Section Waveguide

M.V. Tratnik, Multivariable biorthogonal continuous-discrete Wilson and Racah polynomials

Yisong Yang, Existence of Solutions for a Generalized Yang-Mills Theory

Peter Gritzmann, Laurent Habsieger and Victor Klee, Good and Bad Radii of Convex Polygons

Martin Golubitsky, Martin Krupa and Chjan. C. Lim, Time-Reversibility and Particle Sedimentation

G. Yin, Recent Progress in Parallel Stochastic Approximations

G. Yin, On H-Valued SA: Finite Dimensional Approximations

Chien-Cheng Chang, Accurate Evaluation of the Effect of Diffusion and Conductivity in Certain Equations

Chien-Cheng Chang and Ruey-Ling Chern, The Effect of Viscous Diffusion in Discrete Vortex Dynamics for Slightly Viscous Flows

Li Ta-Tsien (Li Da-qian) and Zhao Yan-Chun, Global Existence of Classical Solutions to the Typical Free Boundary Problem for General Quasilinear Hyperbolic Systems and its Applications

Thierry Cazenave and Fred B. Weissler, The Structure of Solutions to the Pseudo-Conformally Invariant Nonlinear Schrödinger Equation

Marshall Slemrod and Athanasios E. Tzavaras, A Limiting Viscosity Approach for the Riemann Problem in Isentropic Gas Dynamics

Richard D. James and Scott J. Spector, The Formation of Filamentary Voids in Solids

P.J. Vassiliou, On the Geometry of Semi-Linear Hyperbolic Partial Differential Equations in the Plane Integrable by the Method of Darboux

Jerome V. Moloney and Alan C. Newell, Nonlinear Optics

Keti Tenenblat, A Note on Solutions for the Intrinsic Generalized Wave and Sine-Gordon Equations

P. Szmolyan, Heteroclinic Orbits in Singularly Perturbed Differential Equations

Wenxiong Liu, A Parabolic System Arising In Film Development

Daniel B. Dix, Temporal Asymptotic Behavior of Solutions of the Benjamin-Ono-Burgers Equation

Michael Renardy and Yuriko Renardy, On the nature of boundary conditions for flows with moving free surfaces

Werner A. Stahel, Robust Statistics: From an Intellectual Game to a Consumer Product

Avner Friedman and Fernando Reitich, The Stefan Problem with Small Surface Tension

E.G. Kalnins and W. Miller, Jr., Separation of Variables Methods for Systems of Differential Equations in Mathematical Physics

Mitchell Luskin and George R. Sell, The Construction of Inertial Manifolds for Reaction-Diffusion Equations by Elliptic Regularization

Konstantin Mischaikow, Dynamic Phase Transitions: A Connection Matrix Approach

Philippe Le Floch and Li Tatsien, A Global Asymptotic Expansion for the Solution to the Generalized Riemann Problem

Matthew Witten, Ph.D., Computational Biology: An Overview

Matthew Witten, Ph.D., Peering Inside Living Systems: Physiology in a Supercomputer 
Michael Renardy, An existence theorem for model equations resulting from kinetic theories of polymer solutions

Daniel D. Joseph and Luigi Preziosi, Reviews of Modern Physics: Addendum to the Paper "Heat Waves"

Luigi Preziosi, An Invariance Property for the Propagation of Heat and Shear Waves

Gregory M. Constantine and John Bryant, Sequencing of Experiments for Linear and Quadratic Time Effects

Prabir Daripa, On the Computation of the Beltrami Equation in the Complex Plane

A.L. Gorin, D.B. Roe and A.G. Greenberg, On the Complexity of Pattern Recognition Algorithms On a Tree-Structured Parallel Computer

Mark J. Friedman and Eusebius J. Doedel, Numerical computation and continuation of invariant manifolds connecting fixed points

Scott J. Spector, Linear Deformations as Global Minimizers in Nonlinear Elasticity

Denis Serre, Richness and the classification of quasilinear hyperbolic systems

L. Preziosi and F. Rosso, On the stability of the shearing flow between pipes

Avner Friedman and Wenxiong Liu, A system of partial differential equations arising in electrophotography

Jonathan Bell, Avner Friedman, and Andrew A. Lacey, On solutions to a quasilinear diffusion problem from the study of soft tissue

David G. Schaeffer and Michael Shearer, Loss of hyperbolicity in yield vertex plasticity models under nonproportional loading

Herbert C. Kranzer and Barbara Lee Keyfitz, A strictly hyperbolic system of conservation laws admitting singular shocks

S. Laederich and M. Levi, Qualitative dynamics of planar chains

Milan Miklavčič, A sharp condition for existence of an inertial manifold

Charles Collins, David Kinderlehrer, and Mitchell Luskin, Numerical approximation of the solution of a variational problem with a double well potential

Todd Arbogast, Two-phase incompressible flow in a porous medium with various nonhomogeneous boundary conditions

Peter Poláčik, Complicated dynamics in scalar semilinear parabolic equations-in higher space dimension

Bei Hu, Diffusion of penetrant in a polymer: a free boundary problem

Mohamed Sami ElBialy, On the smoothness of the linearization of vector fields near resonant hyperbolic rest points

Max Jodeit, Jr. and Peter J. Olver, On the equation $\operatorname{grad} f=M \operatorname{grad} g$

Shui-Nee Chow, Kening Lu, and Yun-Qiu Shen, Normal form and linearization for quasiperiodic systems

Prabir Daripa, Theory of one dimensional adaptive grid generation

Michael C. Mackey and John G. Milton, Feedback, delays and the origin of blood cell dynamics

D.G. Aronson and S. Kamin, Disappearance of phase in the Stefan problem: one space dimension

Martin Krupa, Bifurcations of relative equilibria

D.D. Joseph, P. Singh, and K. Chen, Couette flows, rollers, emulsions, tall Taylor cells, phase separation and inversion, and a chaotic bubble in Taylor-Couette flow of two immiscible liquids

Artemio González-López, Niky Kamran, and Peter J. Olver, Lie algebras of differential operators in two complex variables

L.E. Fraenkel, On a linear, partly hyperbolic model of viscoelastic flow past a plate

Stephen Schecter and Michael Shearer, Undercompressive shocks for nonstrictly hyperbolic conservation laws

620 Xinfu Chen, Axially symmetric jets of compressible fluid

621 J. David Logan, Wave propagation in a qualitative model of combustion under equilibrium conditions

M.L. Zeeman, Hopf bifurcations in competitive three-dimensional Lotka-Volterra Systems

Allan P. Fordy, Isospectral flows: their Hamiltonian structures, Miura maps and master symmetries

Daniel D. Joseph, John Nelson, Michael Renardy, and Yuriko Renardy, Two-Dimensional cusped interfaces 